\title{
Reaction engineering study of natural gas hydrate formation in special packed -bed reactors by controlling the boundary layer surfaces of the gas -liquid -solid phases
}

Budhijanto Budhijanto

West Virginia University

Follow this and additional works at: https://researchrepository.wvu.edu/etd

\section{Recommended Citation}

Budhijanto, Budhijanto, "Reaction engineering study of natural gas hydrate formation in special packed -bed reactors by controlling the boundary layer surfaces of the gas -liquid -solid phases" (2003). Graduate Theses, Dissertations, and Problem Reports. 2544.

https://researchrepository.wvu.edu/etd/2544

This Dissertation is protected by copyright and/or related rights. It has been brought to you by the The Research Repository @ WVU with permission from the rights-holder(s). You are free to use this Dissertation in any way that is permitted by the copyright and related rights legislation that applies to your use. For other uses you must obtain permission from the rights-holder(s) directly, unless additional rights are indicated by a Creative Commons license in the record and/ or on the work itself. This Dissertation has been accepted for inclusion in WVU Graduate Theses, Dissertations, and Problem Reports collection by an authorized administrator of The Research Repository @ WVU.

For more information, please contact researchrepository@mail.wvu.edu. 


\title{
Reaction Engineering Study of Natural Gas Hydrate Formation in Special Packed-Bed Reactors by Controlling the Boundary Layer Surfaces of the Gas-Liquid-Solid Phases
}

\author{
Budhijanto Budhijanto \\ Dissertation to be submitted to the \\ College of Engineering and Mineral Resources \\ at West Virginia University \\ in partial fulfillment of the requirements \\ for the degree of \\ Doctor of Philosophy \\ in \\ Chemical Engineering
}

\author{
Hisashi O. Kono, Dr. Engineering, Chair \\ Rakesh K. Gupta, Ph.D. \\ John R. Loth, Ph.D. \\ Peter G. Stansberry, Ph.D. \\ Alfred H. Stiller, Ph.D.
}

Department of Chemical Engineering

\author{
Morgantown, West Virginia \\ 2003
}

Keywords: Methane Gas Hydrate, Propane Gas Hydrate, Intrinsic Reaction Rate Constants 


\title{
ABSTRACT \\ Reaction Engineering Study of Natural Gas Hydrate Formation in Special Packed-Bed Reactors by Controlling the Boundary Layer Surfaces of the Gas-Liquid-Solid Phases
}

\author{
Budhijanto Budhijanto
}

Natural gas hydrates (NGH) are crystalline compounds that consist of water and natural gas (NG) molecules. By hydrogen bonds, water molecules form a regular lattice structure containing many cavities. NG molecules occupy the cavities, and therefore stabilize the cavities. NGH are considered to be one of the most potential alternative world energy resources. The applications of $\mathrm{NGH}$ in other fields, such as NG purification, storage, and transportation, are also possible. To obtain the full benefits of all the possible applications of $\mathrm{NGH}$, a full understanding of the kinetics of $\mathrm{NGH}$ formation is needed.

Strictly speaking, the experimental works of reproducible intrinsic chemical reaction rate constants of $\mathrm{NGH}$ formations have not yet been reported. It has been because the control of the interfacial surface areas among the three phases of gas, liquid, and solid involved in the reaction, i.e. NG in gas phase, water in liquid phase, and NGH in solid phase was experimentally difficult during the progress of water-NG conversion into NGH.

In this dissertation, a new experimental method and analysis to determine the intrinsic reaction rate constants of methane gas hydrate $(\mathrm{MGH})$ and propane gas hydrate (PGH) formations are reported. The important aspect of the novel experimental method is the control on the interfacial surface area in the heterogeneous reacting system. The control was achieved using a well-defined specific particle packing structure consisting of spherical, uniform-sized inert particles (e.g. glass beads). The defined packing structure gave reproducible interfacial boundary area of the heterogeneous reacting systems, and the interfacial boundary area can be modeled using simple geometry.

Data showed that initial pressures and glass bead sizes did not affect the reaction rate constants. The data gave the value of $K=(1.89 \pm 0.16) \times 10^{-6} \mathrm{~m} / \mathrm{s}$ for $\mathrm{MGH}$ formation and $K=(2.25 \pm 0.53) \times 10^{-6} \mathrm{~m} / \mathrm{s}$ for PGH formation. Compared to the mass transfer coefficients of methane/propane in water (l) film $k_{L}$ (approximately $10^{-5} \mathrm{~m} / \mathrm{s}$ ), the values of $K$ are significantly smaller for both $\mathrm{MGH}$ and $\mathrm{PGH}$ formations. Hence, the intrinsic reaction rate constants $\left(k_{r}\right)$ of $\mathrm{MGH}$ and $\mathrm{PGH}$ formations were practically represented by the observed $K$. 


\section{Dedicated to My Beloved Daughter}

\section{Dewanggani Kirana}

Although she is far away, her spirit has been a great inspiration and made this journey possible 


\section{ACKNOWLEDGMENT}

I would like to express my sincere gratitude to Professor Hisashi O. Kono for his advices, ideas, enthusiasm, and patience during our discussions and experimental work. My sincere gratitude is also for the members of my examining committee, Professor Rakesh K. Gupta, Professor John R. Loth, Professor Peter G. Stansberry, and Professor Alfred H. Stiller, for their constructive questions and suggestions that were very helpful in completing this dissertation.

I would like to acknowledge QUE Project in Chemical Engineering Department, Gadjah Mada University, Indonesia, for providing me the financial supports to pursue my PhD program in West Virginia University.

My sincere gratitude is also for my wife Wiratni, who has been amazing in organizing everything during her own PhD program. I am also very grateful for her supports, helps, and understanding. I also sincerely thank my family and friends for their supports. In particular, I would like to thank Richard Strobel, Jr., my fellow graduate student, who helped me in problem solving related to experimental equipments.

My special thank is going to James Hall for his invaluable helps in setting up my experiments. I also thank Bonita Helmick, Linda Rogers, and Linda Cox for their patience and help in my paperwork. 


\section{TABLE OF CONTENT}

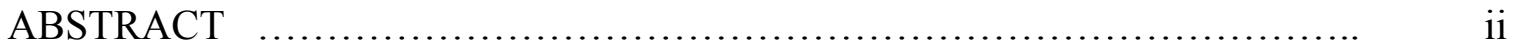

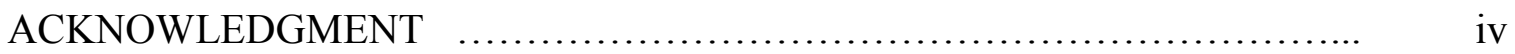

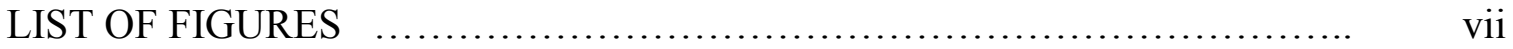

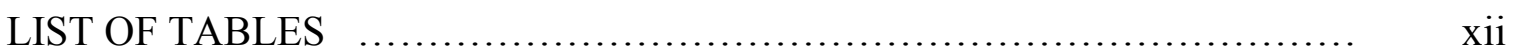

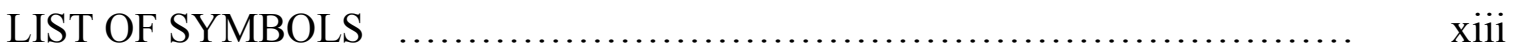

\section{Chapter 1. Introduction}

1.1. Problem $\quad$......................................................... 1

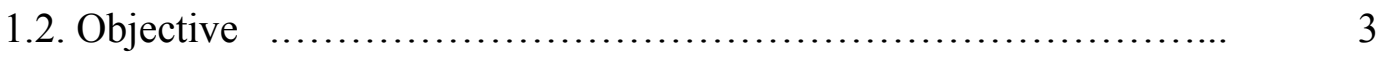

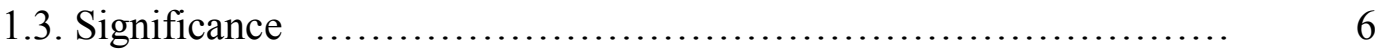

\section{Chapter 2. Literature Review}

2.1. General description of NGH and their structures $\quad \ldots \ldots \ldots \ldots \ldots \ldots . . . . . . . .9$

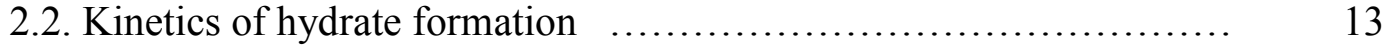

\section{Chapter 3. Physical Model and Relevant Experimental Approach}

3.1. Assumptions and the design experimental conditions $\ldots . . . \ldots \ldots \ldots . .24$

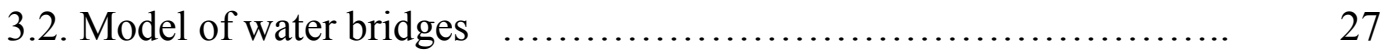

3.3. Physical model of $\mathrm{MGH} / \mathrm{PGH}$ formation $\ldots \ldots \ldots \ldots \ldots \ldots \ldots \ldots \ldots . . \ldots \ldots$

\section{Chapter 4. Experimental}

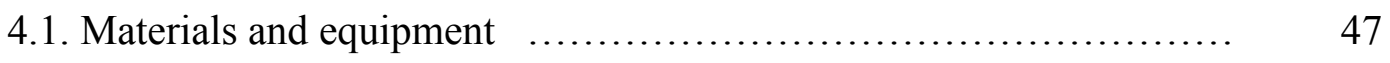

4.2. Bed preparation and gas volume $\left(V_{g}\right)$ measurement $\quad \ldots \ldots \ldots \ldots \ldots \ldots . . . \ldots$

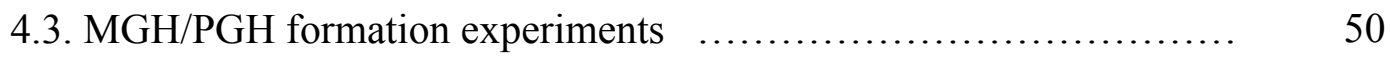


Chapter 5. Results and Discussion

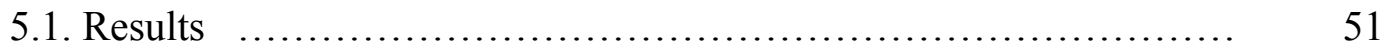

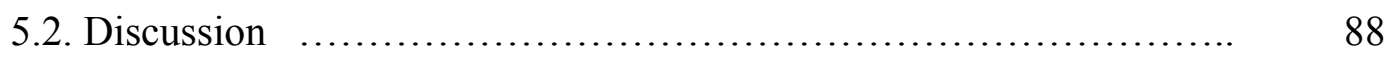

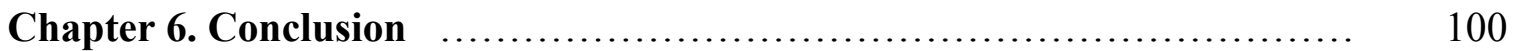

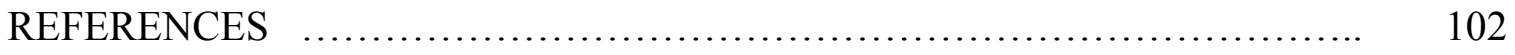

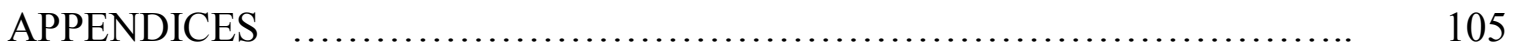

APPENDIX A. Derivations of the Equations of the Surface Area and the

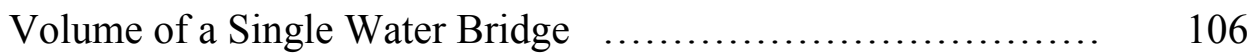

APPENDIX B. Englezos's et al.'s Model and Its Modification $\quad . . \ldots \ldots \ldots \ldots \ldots \ldots . . . . .116$

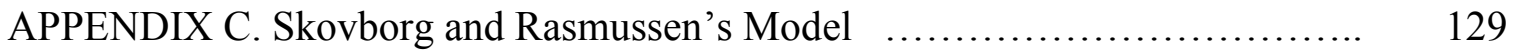

APPENDIX D. Measurements of the Bulk and True Densities of Glass Beads .. 131

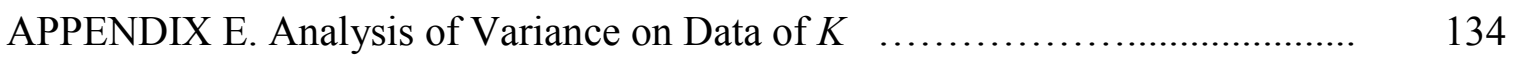




\section{LIST OF FIGURES}

$\begin{array}{lll}\text { Figure No. Figure caption } & \text { Page }\end{array}$

1.1. Schematic pictures of the reactors used in the research on the 4 kinetics of NGH formations: (a) Packed bed reactor used in this work; (b) Semi-batch stirred tank reactor used in the previous works of other researchers.

1.2. Schematic picture of a single water bridge (shown by the shaded 4 area) between two glass beads (shown by the two circles).

2.1. Hydrate crystal unit structures: (a) sI, (b) sII, and (c) sH. 11

2.2. Three hydrate crystal unit structures and the five cavities. 11

3.1. Schematic pictures of the special packed bed reactor with glass 25 beads as the packed bed and water bridges held among the spherical glass beads.

3.2. Model of a single water bridge. 28

3.3. Schematic picture of the packing structure of coordination number 33 8 (orthorhombic packing structure).

3.4. Schematic picture of unit layers of the orthorhombic packing structure: (a) Unit layer of top/bottom side of view; or left/right side of view if the plane of view is rotated as far $30^{\circ}$; (b) Unit layer of the front/back side of view.

3.5. Mass transfer model of MGH/PGH formation. 
Figure No. Figure caption

3.6. Phase diagrams for some simple NG that form NGH. 42

4.1. Experimental equipment for the synthesis of MGH and PGH 47 equipped with $\mathrm{MGH} / \mathrm{PGH}$ dissociation setup.

5.1. Raw data of MGH formation experiments for initial pressure 49.28 bar, $T=273.5 \mathrm{~K}, d_{p}=240 \mu \mathrm{m}$, and $S=0.30$.

5.2. Raw data of MGH formation experiments for initial pressure 42.38 bar, $T=273.5 \mathrm{~K}, d_{p}=240 \mu \mathrm{m}$, and $S=0.30$.

5.3. Raw data of MGH formation experiments for initial pressure 45.83 55 bar, $T=273.5 \mathrm{~K}, d_{p}=240 \mu \mathrm{m}$, and $S=0.30$.

5.4. Raw data of MGH formation experiments for initial pressure 49.28 56 bar, $T=273.5 \mathrm{~K}, d_{p}=510 \mu \mathrm{m}$, and $S=0.30$.

5.5. Evaluation of the MGH formation experiment Run M102 with initial pressure $49.28 \mathrm{bar}, T=273.5 \mathrm{~K}, d_{p}=240 \mu \mathrm{m}$, and $S=0.30$.

5.6. Evaluation of the MGH formation experiment Run M103 with initial pressure 49.28 bar, $T=273.5 \mathrm{~K}, d_{p}=240 \mu \mathrm{m}$, and $S=0.30$.

5.7. Evaluation of the MGH formation experiment Run M104 with initial pressure $49.28 \mathrm{bar}, T=273.5 \mathrm{~K}, d_{p}=240 \mu \mathrm{m}$, and $S=0.30$.

5.8. Evaluation of the MGH formation experiment Run M105 with initial pressure $49.28 \mathrm{bar}, T=273.5 \mathrm{~K}, d_{p}=240 \mu \mathrm{m}$, and $S=0.30$.

5.9. Evaluation of the MGH formation experiment Run M106 with 61 initial pressure 49.28 bar, $T=273.5 \mathrm{~K}, d_{p}=240 \mu \mathrm{m}$, and $S=0.30$. 
Figure No. Figure caption

5.10. Evaluation of the MGH formation experiment Run M107 with initial pressure $49.28 \mathrm{bar}, T=273.5 \mathrm{~K}, d_{p}=240 \mu \mathrm{m}$, and $S=0.30$.

5.11. Evaluation of the MGH formation experiment Run M108 with 63 initial pressure $49.28 \mathrm{bar}, T=273.5 \mathrm{~K}, d_{p}=240 \mu \mathrm{m}$, and $S=0.30$.

5.12. Evaluation of the MGH formation experiment Run M109 with 64 initial pressure $42.38 \mathrm{bar}, T=273.5 \mathrm{~K}, d_{p}=240 \mu \mathrm{m}$, and $S=0.30$.

5.13. Evaluation of the MGH formation experiment Run M110 with 65 initial pressure $42.38 \mathrm{bar}, T=273.5 \mathrm{~K}, d_{p}=240 \mu \mathrm{m}$, and $S=0.30$.

5.14. Evaluation of the MGH formation experiment Run M111 with 66 initial pressure $42.38 \mathrm{bar}, T=273.5 \mathrm{~K}, d_{p}=240 \mu \mathrm{m}$, and $S=0.30$.

5.15. Evaluation of the MGH formation experiment Run M112 with initial pressure $45.83 \mathrm{bar}, T=273.5 \mathrm{~K}, d_{p}=240 \mu \mathrm{m}$, and $S=0.30$.

5.16. Evaluation of the MGH formation experiment Run M113 with 68 initial pressure $45.83 \mathrm{bar}, T=273.5 \mathrm{~K}, d_{p}=240 \mu \mathrm{m}$, and $S=0.30$.

5.17. Evaluation of the MGH formation experiment Run M114 with 69 initial pressure $45.83 \mathrm{bar}, T=273.5 \mathrm{~K}, d_{p}=240 \mu \mathrm{m}$, and $S=0.30$.

5.18. Evaluation of the MGH formation experiment Run M115 with 70 initial pressure $49.28 \mathrm{bar}, T=273.5 \mathrm{~K}, d_{p}=510 \mu \mathrm{m}$, and $S=0.30$.

5.19. Evaluation of the MGH formation experiment Run M116 with initial pressure $49.28 \mathrm{bar}, T=273.5 \mathrm{~K}, d_{p}=510 \mu \mathrm{m}$, and $S=0.30$.

5.20. Evaluation of the MGH formation experiment Run M117 with 72 initial pressure $49.28 \mathrm{bar}, T=273.5 \mathrm{~K}, d_{p}=510 \mu \mathrm{m}$, and $S=0.30$. 
Figure No. Figure caption

5.21. Evaluation of the MGH formation experiment Run M118 with 73 initial pressure $49.28 \mathrm{bar}, T=273.5 \mathrm{~K}, d_{p}=510 \mu \mathrm{m}$, and $S=0.30$.

5.22. Evaluation of the MGH formation experiment Run M119 with 74 initial pressure $49.28 \mathrm{bar}, T=273.5 \mathrm{~K}, d_{p}=510 \mu \mathrm{m}$, and $S=0.30$.

5.23. Evaluation of the MGH formation experiment Run M120 with 75 initial pressure $49.28 \mathrm{bar}, T=273.5 \mathrm{~K}, d_{p}=510 \mu \mathrm{m}$, and $S=0.30$.

5.24. Raw data of PGH formation experiments for initial pressure 4.32 bar, $T=273.5 \mathrm{~K}, d_{p}=240 \mu \mathrm{m}$, and $S=0.30$.

5.25. Raw data of PGH formation experiments for initial pressure 4.18 bar, $T=273.5 \mathrm{~K}, d_{p}=240 \mu \mathrm{m}$, and $S=0.30$.

5.26. Evaluation of the PGH formation experiment Run P101 with initial pressure $4.32 \mathrm{bar}, T=273.5 \mathrm{~K}, d_{p}=240 \mu \mathrm{m}$, and $S=0.30$.

5.27. Evaluation of the PGH formation experiment Run P102 with initial pressure $4.32 \mathrm{bar}, T=273.5 \mathrm{~K}, d_{p}=240 \mu \mathrm{m}$, and $S=0.30$.

5.28. Evaluation of the PGH formation experiment Run P103 with 82 initial pressure $4.32 \mathrm{bar}, T=273.5 \mathrm{~K}, d_{p}=240 \mu \mathrm{m}$, and $S=0.30$.

5.29. Evaluation of the PGH formation experiment Run P106 with 83 initial pressure 4.18 bar, $T=273.5 \mathrm{~K}, d_{p}=240 \mu \mathrm{m}$, and $S=0.30$.

5.30. Evaluation of the PGH formation experiment Run P107 with 84 initial pressure $4.18 \mathrm{bar}, T=273.5 \mathrm{~K}, d_{p}=240 \mu \mathrm{m}$, and $S=0.30$.

5.31. Evaluation of the PGH formation experiment Run P108 with 85 initial pressure $4.18 \mathrm{bar}, T=273.5 \mathrm{~K}, d_{p}=240 \mu \mathrm{m}$, and $S=0.30$. 
$\begin{array}{lll}\text { Figure No. Figure caption } & \text { Page }\end{array}$

5.32. Effect of mixing on $K^{*}$ of Englezos et al.'s experiments (Figure 1396 in Englezos et al.’s paper (1987a)).

$\begin{array}{lll}\text { A.1. } & \text { Model of a single water bridge. } & 106\end{array}$

$\begin{array}{lll}\text { A.2. } & \text { Curve of } y=f(x) . & 107\end{array}$

A.3. Geometry of the model of a single water bridge. 108

A.4. Detail of the geometry of the model of a single water bridge. 109

A.5. Geometry of a half wetted surface of a glass bead particle. 114

$\begin{array}{lll}\text { B.1. } & \text { Model of NGH crystal growth. }\end{array}$ 


\section{LIST OF TABLES}

$\begin{array}{lll}\text { Table No. Table caption } & \text { Page }\end{array}$

1.1 NGH Equilibrium Compositions (\% by volume) $\quad 7$

3.1. Glass Bead Bulk Density $\left(\rho_{b}\right)$ and True Density $\left(\rho_{s}\right)$, and the 30

Corresponding Porosity of the Bed $(\varepsilon)$ and Coordination Number

3.2. Relative Saturation of Void by Water $S$ at Two Values of $2 \theta \quad 35$

3.3 Parameters in the Model of a Single Water Bridge 35

3.4 Data of acentric factor $(\omega)$, critical pressure $\left(P_{c}\right)$ and temperature 41 $\left(T_{c}\right)$

4.1. Experimental Data of Bed Preparation and Gas Volume $\left(V_{g}\right) \quad 49$ Measurement

5.1. Evaluation of $K$ for MGH Formation Experimental Data 76

5.2. $\quad$ Evaluation of $K$ for PGH Formation Experimental Data 77

5.3. $K$ of $\mathrm{MGH}$ in the Growth Period with the Apparent Hydration 87 Number $\left(n_{w, \text { app }}\right)$ and the Water Conversion when the Data Depart from the Linear Line of the Model $\left(X_{H 2 O}\right)$

5.4. $K$ of PGH in the Growth Period with the Apparent Hydration 88 Number $\left(n_{w, \text { app }}\right)$ and the Water Conversion when the Data Depart from the Linear Line of the Model $\left(X_{H 2 O}\right)$

D.1. Measurements of Glass Bead Bulk Density

D.2. Measurements of Glass Bead True Density 


\section{LIST OF SYMBOLS}

$A \quad=$ lumped pre-exponential constant in the Vysniauskas and Bishnoi's model of Equation $(2.1)\left(\mathrm{cm}^{3} / \mathrm{cm}^{2} \cdot \min \cdot \mathrm{bar}^{2}\right)$

$A \quad=$ total surface area of NG $(\mathrm{g})-$ water $(1)$ interface $\left(\mathrm{m}^{2}\right)$

$A \quad=$ uncorrected group $S S$ in ANOVA test

$A_{(g-l)} \quad=$ gas-liquid interfacial area in Skovborg-Rasmussen's model of Equation $(2.5)\left(\mathrm{m}^{2}\right)$

$A_{p} \quad=$ surface area of NGH particle in Englezos et al.'s model of Equation $(2.2)\left(\mathrm{m}^{2}\right)$

$A_{\text {single }} \quad=$ surface area of a single water bridge $\left(\mathrm{m}^{2}\right)$

$a \quad=$ empirical parameter in the Vysniauskas and Bishnoi's model of Equation $(2.1)\left(\mathrm{K}^{\mathrm{b}}\right)$

$a \quad=$ radius of glass beads $(\mathrm{m})$

$a_{s} \quad=$ the effective surface area of gas-water interface in the Vysniauskas and Bishnoi's model of Equation (2.1) $\left(\mathrm{cm}^{2}\right)$

$a^{*} \quad=$ gas-liquid interfacial area per unit of liquid volume $\left(\mathrm{m}^{2} / \mathrm{m}^{3}\right)$

$b \quad=$ empirical parameter in the Vysniauskas and Bishnoi's model of

\section{Equation (2.1) (-)}

$b_{1} \quad=$ the amount of water in $1 \mathrm{~cm}^{3}$ of $\mathrm{MGH}(\mathrm{g})$ 


$$
\begin{aligned}
& b_{2}=\text { the amount of methane in } 1 \mathrm{~cm}^{3} \text { of MGH }(\mathrm{g}) \\
& \text { C } \quad \text { coordination number }(-) \\
& C_{g}, C_{g, 0}, C_{g}^{e q}=\text { concentration of } \mathrm{NG} \text { in gas phase, } C_{g} \text { at } t=0, C_{g} \text { at equilibrium } \\
& \left(\mathrm{mol} / \mathrm{m}^{3}\right) \\
& C_{g, \text { end }}=\text { concentration of } \mathrm{NG} \text { in the gas phase at the end of reaction }\left(\mathrm{mol} / \mathrm{m}^{3}\right) \\
& C_{i} \quad=\text { concentration of dissolved NG in water (1) phase at water (1)-NGH (s) } \\
& \text { interface }\left(\mathrm{mol} / \mathrm{m}^{3}\right) \\
& C_{1}, C_{2} \quad=\text { integration constants of Equation (B.13) (MPa) } \\
& C^{e q}=\text { equilibrium concentration of dissolved NG in water (1) phase at water } \\
& \text { (1)-NGH (s) interface }\left(\mathrm{mol} / \mathrm{m}^{3}\right) \\
& C^{*} \quad=\text { concentration of dissolved NG in water (1) phase at the gas-water (1) } \\
& \text { interface, }\left(\mathrm{mol} / \mathrm{m}^{3}\right) \\
& \mathrm{CF} \quad=\text { correction factor in ANOVA test } \\
& c, c_{e q}=\text { concentration of dissolved NG in water (l) in Englezos et al.'s model of } \\
& \text { Equation (5.4), } c \text { at three-phase equilibrium condition }\left(\mathrm{mol} / \mathrm{m}^{3}\right) \\
& c_{w 0} \quad=\text { initial concentration of water molecules, }\left(\mathrm{mol} / \mathrm{m}^{3}\right) \\
& D_{L}, D_{L, T i} \quad=\text { diffusion coefficient of dissolved NG in water (1) phase, } D_{L} \text { at } \\
& \text { temperature } T_{i}\left(\mathrm{~m}^{2} / \mathrm{s}\right) \\
& D^{*} \quad \equiv \frac{D_{L} c_{w 0}}{H^{*}}=\text { combined rate parameter in Englezos et al.'s model of }
\end{aligned}
$$$$
\text { Equation (B.10) (mol/m.s.MPa) }
$$ 


$$
\begin{aligned}
& d_{p} \quad=\text { glass bead diameter }(\mu \mathrm{m}) \\
& d f \quad=\text { degree of freedom in ANOVA test } \\
& \Delta E_{a} \quad=\text { the apparent energy activation of MGH or EGH formation in the } \\
& \text { Vysniauskas and Bishnoi's model of Equation (2.1) (kJ/mol) } \\
& F, F_{\alpha, d f 1, d f 2}=F \text { value in ANOVA test, critical } F \text { value } \\
& f, f_{e q}, f_{i} \quad=\text { fugacity of the NG in Englezos et al.'s model of Equation (2.2), } f \text { at } \\
& \text { three-phase equilibrium condition; } f \text { at NGH crystal-liquid interface } \\
& \text { condition }(\mathrm{MPa}) \\
& f_{b} \quad=\text { fugacity of the NG at the condition of the bulk of the liquid phase (MPa) } \\
& f_{g} \quad=\text { fugacity of the NG at the condition of the gas phase }(\mathrm{MPa}) \\
& G, G_{a v g}, G_{b} \quad=\text { the linear growth rate of NGH crystals, average } G, G \text { in the bulk of } \\
& \text { liquid }(\mathrm{m} / \mathrm{s}) \\
& \text { g } \quad \text { gas } \\
& \Delta g \quad=\text { free energy change per unit volume of } \mathrm{NGH}\left(\mathrm{J} / \mathrm{m}^{3}\right) \\
& H \quad=\text { partition factor (Henry's constant) of dissolved NG in water (1) at the } \\
& \text { temperature of reaction }(-) \\
& H^{*} \quad=\text { partition factor (Henry's constant) of dissolved NG in water (1) at the } \\
& \text { temperature of reaction ( } \mathrm{atm} / \mathrm{mole} \text { fraction) or }(\mathrm{kPa} / \mathrm{mole} \text { fraction) or } \\
& \text { ( } \mathrm{MPa} / \text { mole fraction) } \\
& (J)_{y=0},(J)_{y=\mathrm{yL}}=\text { molar flux at gas-liquid interface, } J \text { at } y=y_{L}\left(\mathrm{~mol} / \mathrm{m}^{2} \cdot \mathrm{s}\right)
\end{aligned}
$$




$$
\begin{aligned}
& K=\text { overall reaction rate constant }(\mathrm{m} / \mathrm{s}) \\
& K^{*} \quad=\text { combined rate parameter in Englezos et al.'s model of Equation (2.2) } \\
& \left(\mathrm{mol} / \mathrm{m}^{2} \cdot \mathrm{s} \cdot \mathrm{MPa}\right) \\
& k_{d} \quad=\text { mass transfer coefficient of the dissolved NG around the NGH } \\
& \text { crystals in the Englezos et al.'s model of Equation }(2.3)\left(\mathrm{mol} / \mathrm{m}^{2} \cdot \mathrm{s} \cdot \mathrm{MPa}\right) \\
& k_{f} \quad=\text { overall reaction rate constant of MGH formation in the Kono et al's } \\
& \text { model of Equation (2.6), }\left(\mathrm{mol}^{\left(1-\mathrm{n}^{*}\right)} / \mathrm{min}\right) \\
& k_{L} \quad=\text { mass transfer coefficient of } \mathrm{NG} \text { in water }(1) \text { film }(\mathrm{m} / \mathrm{s}) \\
& k_{r} \quad=\text { intrinsic reaction rate constant in the Englezos et al.'s model of Equation } \\
& \text { (2.3) }\left(\mathrm{mol} / \mathrm{m}^{2} \cdot \mathrm{s} \cdot \mathrm{MPa}\right) \\
& k_{r} \quad=\text { intrinsic reaction rate constant of NGH formation }(\mathrm{m} / \mathrm{s}) \\
& L \quad=\text { length of the curve in Figure A.2 (unit length) } \\
& L \quad=\text { distance between the gas-liquid interface and the bottom of the reactor } \\
& \text { in Englezos et al.'s model of Equation (B.32) (m) } \\
& 1 \quad=\text { liquid } \\
& M \quad=\text { moisture content of the bed of glass beads }(-) \\
& M_{H} \quad=\text { molecular weight of NGH of the form } \mathrm{X} \cdot \mathrm{n}_{\mathrm{w}} \mathrm{H}_{2} \mathrm{O} \text { with } \mathrm{X} \text { represent } \\
& \text { species of NG in the NGH }(\mathrm{g} / \mathrm{mol}) \\
& M_{H 2 O}=\text { molecular weight of water }(=18.016 \mathrm{~g} / \mathrm{mol})
\end{aligned}
$$


$\begin{aligned} M S, M S_{a}, M S_{e}= & \text { mean squares in ANOVA test, } M S \text { among groups, } M S \text { of error } \\ N & =\text { total number of water bridges inside the bed of glass beads (-) } \\ N & =\text { total number of data in ANOVA test (-) } \\ N_{g b} & =\text { number of glass bead particles in the bed (-) } \\ N_{p} & =\text { number of NGH particles (-) } \\ N G \quad & \text { total number of NG species that formed NGH in the Skovborg and } \\ & \text { Rasmussen's model of Equation (C.2) (-) } \\ n & =\text { moles of the NG consumed in the Englezos et al.'s model of Equation } \\ & \text { (2.2) and Skovborg and Rasmussen's model of Equation (2.5) (mol) } \\ n & =\text { moles of NG in gas phase (mol) } \\ n_{C H_{4}} & \text { moles of methane in the gas phase according to Kono et al's model of }\end{aligned}$

Equation (2.6) (mol)

$n_{e q} \quad=$ amount of NG that would have been dissolved at the equilibrium pressure of NGH formation and temperature of experiment (mol)

$n_{i} \quad=$ total number of data of condition $i$ in ANOVA test

$n_{t b} \quad=$ the measured number of moles of NG that have been dissolved at the turbidity point (mol)

$n_{\text {tot }} \quad=$ total moles of NG consumed at a given time in the Skovborg and Rasmussen's model of Equation (C.2) (mol)

$n_{w}, n_{w, a p p .} \quad=$ hydration number, apparent $n_{w}(-)$

$n^{*} \quad=$ overall order of reaction in the Kono et al.'s model of Equation (2.6) (-)

$P \quad=$ gas pressure $(\mathrm{MPa})$ or $($ bar $)$ 


$$
\begin{array}{ll}
P_{c} & =\text { critical pressure of NG (bar) } \\
P_{r} & =\text { reduced pressure }(-) \\
P^{e q} & =\text { equilibrium pressure of NGH formation at temperature of reaction }
\end{array}
$$

$$
\begin{array}{ll}
R & =\text { ideal gas constant }\left(=8.314 \times 10^{-5} \mathrm{~m}^{3} \cdot \mathrm{bar} / \mathrm{mol} \cdot \mathrm{K}=8.314 \mathrm{~J} / \mathrm{mol} \cdot \mathrm{K}\right) \\
R_{y}(t) \quad & \text { total reaction rate of NGH formation in Englezos et al.'s model } \\
& \left(\mathrm{mol} / \mathrm{m}^{3} \cdot \mathrm{s}\right) \\
& =
\end{array}
$$$$
\text { Figures (5.5) to (5.23) and (5.26) to (5.31) (-) }
$$$$
r \quad=\text { the consumption rate of methane or ethane in the Vysniauskas and }
$$$$
\text { Bishnoi's model of Equation }(2.1)\left(\mathrm{cm}^{3} / \mathrm{min}\right)
$$$$
r, r_{c r} \quad=\text { radius of NGH crystals in Englezos et al.'s model, critical } r(\mathrm{~m})
$$$$
r_{1} \quad=\text { radius of the meniscus of a water bridge }(\mathrm{m})
$$$$
r_{2} \quad=\text { the shortest distance between the contact point of two glass beads }
$$$$
\text { and the meniscus of its water bridge }(\mathrm{m})
$$$$
S \quad \text { = relative saturation of packed bed void by water (-) }
$$$$
\text { SS } \quad=\text { sum of squares in ANOVA test }
$$$$
s \quad=\text { diameter of the NGH crystal }(\mathrm{m})
$$$$
\mathrm{s} \quad=\text { solid }
$$$$
T \quad=\text { temperature of reaction }(\mathrm{K})
$$ 


$$
\begin{aligned}
& T \quad=\text { uncorrected total } S S \text { in ANOVA test } \\
& T_{c} \quad=\text { critical temperature of } \mathrm{NG}(\mathrm{K}) \\
& T_{r} \quad=\text { reduced temperature }(-) \\
& \Delta T \quad=\text { degree of super-cooling in the Vysniauskas and Bishnoi's model of } \\
& \text { Equation (2.1) (K) } \\
& t \quad=\text { time of reaction }(\mathrm{s}) \text { or }(\mathrm{min}) \\
& t_{\alpha, d f} \quad=\text { critical } \mathrm{t} \text { value in statistical analysis } \\
& V_{g} \quad=\text { volume of gas phase inside the reactor }\left(\mathrm{m}^{3}\right) \\
& V_{g b} \quad=\text { volume of glass beads }(\mathrm{mL}) \\
& V_{L} \quad=\text { liquid volume in the Englezos et al.'s model of Equation (B.40) }\left(\mathrm{m}^{3}\right) \\
& V_{\text {pic }} \quad=\text { volume of a picnometer }(\mathrm{mL}) \\
& V_{\text {revolution }}=\text { volume of solid of revolution (unit volume) } \\
& V_{\text {single }} \quad=\text { volume of a single water bridge }\left(\mathrm{m}^{3}\right) \\
& V_{1}, V_{2} \quad=\text { volume of solids of revolution in derivation of the equation of } V_{\text {single }} \\
& \text { (unit volume) } \\
& \Delta V \quad=\text { volume of gas released to a graduated cylinder in } V_{g} \text { measurement }(\mathrm{mL}) \\
& W \quad=\text { total weight of glass beads in the bed }(\mathrm{kg}) \text { or }(\mathrm{g}) \\
& W_{\mathrm{H} 2 \mathrm{O}}=\text { water weight }(\mathrm{g}) \\
& W_{0}, W_{1}, W_{2}, W_{3}, W_{4} \quad=\text { weight of samples in the measurement of } \rho_{b} \text { and } \rho_{s}(\mathrm{~g})
\end{aligned}
$$


$w$ $=$ variable in geometric substitution of $x$ in derivation of the equations of $A_{\text {single }}$ and $V_{\text {single }}($ radian)

$X_{\mathrm{H} 2 \mathrm{O}}=$ conversion of water into $\mathrm{NGH}(-)$

$x, x^{*}$

$x$

$x$

Y

$\equiv f-f_{\text {eq }}=$ driving force of NGH formation reaction in Englezos et al.'s

model (MPa)

$y \quad \equiv \ln \left(\frac{C_{g}-C_{g}^{e q}}{C_{g, 0}-C_{g}^{e q}}\right)$ in the regression equations presented in Figures (5.5) to

(5.23) and (5.26) to (5.31) (-)

$y=$ ordinate of rectangular coordinate

$y_{L} \quad=$ water (1) film thickness $(\mathrm{m})$

$Z^{0}, Z^{1} \quad=$ parameters in the Lee/Kesler compressibility factor correlation of 


$$
\begin{array}{ll} 
& \text { Equation (3.23) }(-) \\
z & =\text { compressibility factor }(-)
\end{array}
$$

\section{Greek letters}

$$
\begin{aligned}
& \alpha_{2} \quad=\text { nucleation rate constant }\left(\text { nuclei } / \mathrm{m}^{2} \cdot \mathrm{s}\right) \\
& \beta=\text { secondary nucleation rate per unit volume }\left(\text { nuclei } / \mathrm{m}^{3} \cdot \mathrm{s}\right) \\
& \gamma \quad=\text { overall order of reaction with respect to pressure in the Vysniauskas } \\
& \text { and Bishnoi's model of Equation (2.1) (-) } \\
& \delta(r-0) \quad=\text { dirac delta function }\left(\mathrm{m}^{-1}\right) \\
& \delta_{\text {om }} \quad=\text { Kronecker delta }\left(\mathrm{m}^{m}\right) \\
& \gamma \quad \equiv y_{L} \sqrt{\frac{4 \pi K^{*} \mu_{2}}{D^{*}}}=\text { Hatta number }(-) \\
& \varepsilon \quad=\text { porosity of the bed }(-) \\
& \theta \quad=\text { angle as defined in Figure } 3.2 \text { (radian) } \\
& \mu_{n} \quad=\text { the } n \text {-th moment of crystal size distribution }\left(\mathrm{m}^{\mathrm{n}} / \mathrm{m}^{3}\right) \\
& \mu_{0}^{0} \quad=\text { number of NGH particles per unit volume of liquid phase at } t=0 \\
& \left(\text { nuclei } / \mathrm{m}^{3}\right) \\
& \mu_{n}^{0} \quad=\text { the initial conditions of } \mu_{n}\left(\mathrm{~m}^{n} / \mathrm{m}^{3}\right) \\
& v_{m} \quad=\text { molar volume of } \mathrm{NGH}\left(\mathrm{m}^{3} / \mathrm{mol}\right)
\end{aligned}
$$




$$
\begin{array}{ll}
v_{w} & =\text { molar volume of water }\left(\mathrm{m}^{3} / \mathrm{mol}\right) \\
\rho_{b} & =\text { bulk density of glass beads }\left(\mathrm{kg} / \mathrm{m}^{3}\right) \text { or }(\mathrm{g} / \mathrm{mL}) \\
\rho_{H} & =\text { NGH density }\left(\mathrm{kg} / \mathrm{m}^{3}\right) \text { or }\left(\mathrm{g} / \mathrm{m}^{3}\right) \\
\rho_{s} & =\text { true density of glass beads }\left(\mathrm{kg} / \mathrm{m}^{3}\right) \text { or }(\mathrm{g} / \mathrm{mL}) \\
\rho_{w} & =\text { water }(1) \text { density }\left(\mathrm{kg} / \mathrm{m}^{3}\right) \\
\sigma & =\text { surface tension of NGH in water }\left(\mathrm{J} / \mathrm{m}^{2}\right) \\
\phi(r, t) & =\text { crystal size distribution }\left(\mathrm{m}^{-4}\right) \\
\omega & =\text { acentric factor }(-) \\
\psi(r, t) d r & =\text { fraction of the crystals born in the size range from } r \text { to } r+d r(-)
\end{array}
$$

\section{Subscripts/superscripts}

$$
\begin{array}{ll}
\operatorname{lvg} \quad & \text { average value } \\
i & =\text { components of NG mixtures in the Skovborg and Rasmussen's model } \\
& \text { of Equation (C.2) } \\
= & \text { data of condition } i \text { in the ANOVA test } \\
= & \text { gaseous component (methane and ethane) in the Englezos et al.'s model } \\
j \quad & \text { of Equation (2.4) } \\
= & j \text {-th datum in the ANOVA test } \\
j \quad & \text { particle in the Englezos et al.'s model of Equation (2.2) } \\
p \quad &
\end{array}
$$




\section{CHAPTER 1 \\ INTRODUCTION}

\subsection{Problem}

Natural gas hydrates $(\mathrm{NGH})$ are crystalline clathrate compounds that consist of water and natural gas (NG) molecules. The water molecules form cavities in which $\mathrm{NG}$ molecules are trapped. The NG molecules stabilize the crystalline compounds, enabling them to form above the freezing point of water. The formation of NGH caused serious problems in the oil and gas industry, as NGH crystals blocked gas transportation lines. The problems attracted research on NGH. In the 1960s, Russian scientists proved that NG can combine with water under certain thermodynamic condition in the earth to form NGH deposits. In the later year, it was found that NG deposits in hydrate phase were by two orders of magnitude greater than the world's explored NG reserves (Makogon, 1997). Since then, NGH is considered as one of the most potential alternative world energy resources.

Exploitation of $\mathrm{NGH}$ resources needs a full understanding of $\mathrm{NGH}$ formation. From thermodynamic point of view, NGH formation has been successfully modeled. It is the kinetics of $\mathrm{NGH}$ formation that still needs more attention, especially the determination of the intrinsic reaction rate constants.

Some experimental works on NGH formation have been done in the past by some researchers. Vysniauskas and Bishnoi $(1983,1985)$ reported the kinetics data of the methane gas hydrate $(\mathrm{MGH})$ and ethane gas hydrate $(\mathrm{EGH})$ formations. They did their experiments in a semi batch reactor at isothermal and isobaric conditions. A semi 
empirical model was formulated to correlate the data. Englezos et al. (1987a, b) proposed a kinetics model of the MGH and EGH formations based on crystallization theory. They modified the experimental procedure of Vysniauskas and Bishnoi to get better experimental data. Dholabai et al. (1993) used the Englezos et al.'s model to study the kinetics of MGH formation in aqueous electrolyte solutions. Skovborg and Rasmussen (1994) simplified Englezos et al.'s model to take into account mass transfer effects only. Malegaonkar et al. (1997) used the Englezos et al.'s model to study the kinetics of carbon dioxide hydrate and MGH formation. Kono et al. (2002) used a packed bed reactor to study the kinetics of MGH formation and decomposition. They used glass beads as the bed of the reactor. A simple kinetics model was proposed to determine the MGH formation rate constants. All these past experimental works will be discussed more in the literature review.

Among those experimental works, the ones that were considered to be the two most important pioneering works on the kinetics of $\mathrm{NGH}$ formations were done by Englezos et al. (1987a, b) and Skovborg and Rasmussen (1994). In their attempt to determine the intrinsic reaction rate of NGH formations, they used semi batch stirred tank reactors at isothermal and isobaric conditions. Using the reacting system, Englezos et al. found that the overall reaction was controlled by the intrinsic NGH formation reaction. Using the same system at the same operating condition, Skovborg and Rasmussen argued that the overall reaction was controlled by the mass transfer of dissolved NG from gaswater liquid interface to the bulk of the water liquid. One important drawback of their system was the fact that there was no control on the contacts among the three phases involved in the reaction, i.e. NG in the gas phase, water in liquid phase, and NGH in solid 
phase. While Skovborg and Rasmussen's model ${ }^{1}$ is relatively simple, Englezos et al.'s model $^{2}$ contains many parameters that are not easily measured nor predicted, such as the number, the size, and the surface area of NGH crystals.

\subsection{Objective}

The objective of this research is to introduce a new method to determine the intrinsic reaction rate constants of $\mathrm{NGH}$ formations, i.e. $\mathrm{MGH}$ and propane gas hydrate (PGH) formations. $\mathrm{MGH}$ and $\mathrm{PGH}$ are two important kinds of $\mathrm{NGH}$. The schematic picture of the reactor used in the experiments is shown in Figure 1.1. (a). Based on the reacting system used in the experiments, i.e. a novel packed-bed reactor, an appropriate physical model that enabled us to experimentally measure the intrinsic reaction rate constants was proposed. To test the proposed model, series of experiments were done under selected pressure and temperature such that no ice was formed, no NG was condensed, and insignificant amount of water reacted during NG injection. The reacting system was designed such that the surface area of contacts among the three phases involved in the reaction, i.e. NG, liquid water, and solid NGH was large. It was achieved by utilization of inert, spherical, uniformly-sized particles (e.g. glass beads) to create tiny units of reactor in form of water bridges on the points of contacts among glass beads. The schematic picture of a single water bridge is shown in Figure 1.2. The proposed physical model was then called water-bridge-dispersion-model. All the experiments were done under isothermal conditions to measure the reaction rate of NGH formations. Then, the reaction rate constants could be calculated based on the proposed physical model.

\footnotetext{
${ }^{1}$ See Appendix C for the derivation of the Skovborg and Rasmussen's model.

${ }^{2}$ See Appendix B for the derivation of the Englezos et al.'s model.
} 


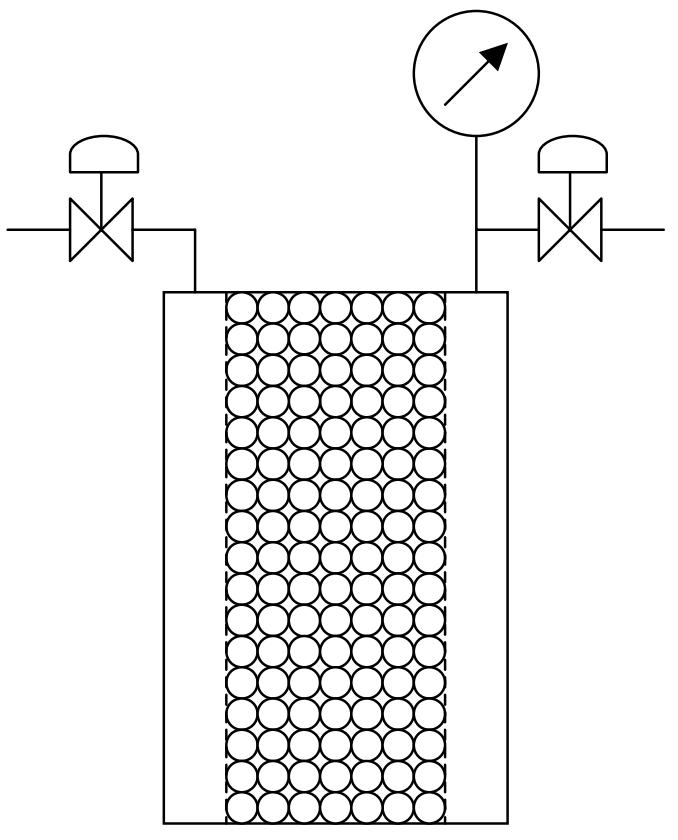

(a)

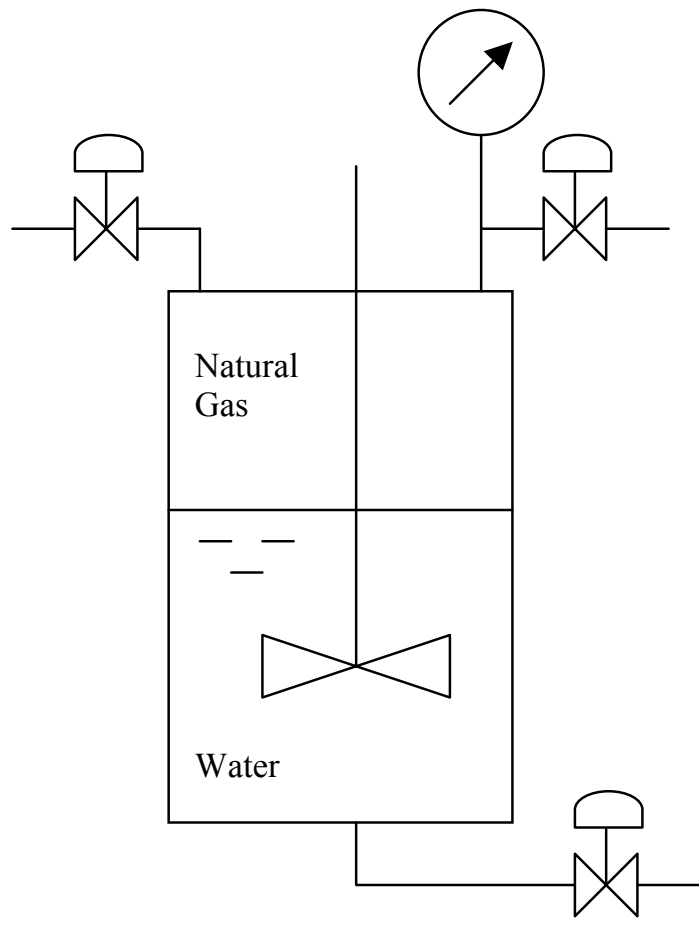

(b)

Figure 1.1. Schematic pictures of the reactors used in the research on the kinetics of NGH formations: (a) Packed bed reactor used in this work; (b) Semi-batch stirred tank reactor used in the previous works of other researchers.

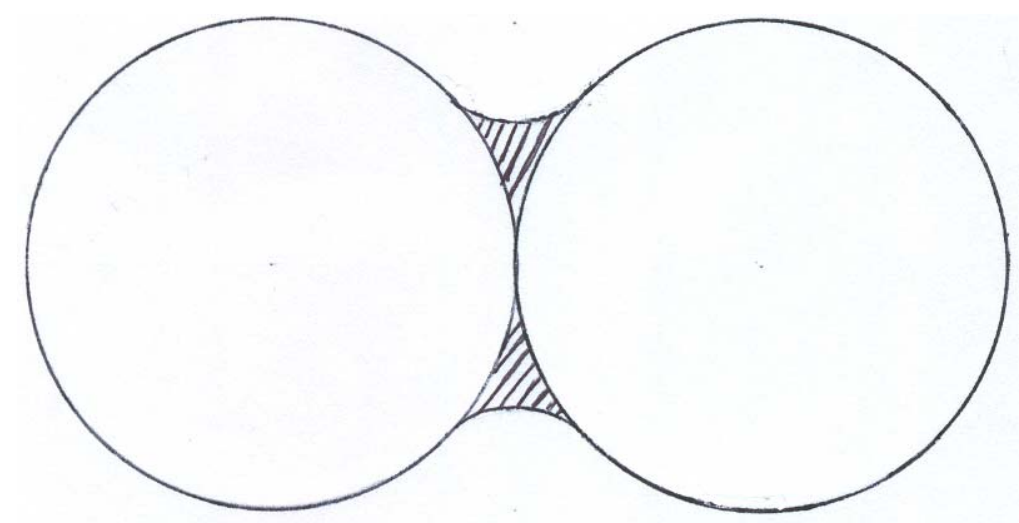

Figure 1.2. Schematic picture of a single water bridge (shown by the shaded area) between two glass beads (shown by the two circles). 
We found that the water bridges existing among the glass beads in the welldefined specific particle packing structures could be well controlled by adjusting the glass bead size $\left(d_{p}\right)$ and the relative saturation of the packed bed void by water $(S)$, where $S$ means the ratio of water (1) volume to the volume that is not occupied by glass beads in the bed. By selecting uniformly sized glass beads $\left(d_{p}\right)$ and $S$, the packed bed packing structure and the distribution of water inside it could be predicted; and the inter boundary surfaces of the above participating reactants among the well-defined, chemically inert, spherical, uniformly sized glass beads could be controlled. The well-defined packing structures gave reproducible reaction conditions, and data obtained were reproducible too. Based on the data, the kinetics of NGH formations was determined.

The most important difference between the semi-batch stirred tank reactors used by other researchers (shown in Figure 1.1. (b)) and our packed bed reactor (shown in Figure 1.1. (a)) is that in the packed bed reactor, the large boundary surface area of contacts among NG-water-NGH could be provided without mechanical stirring. No use of mechanical stirring makes it possible to control the inter boundary surfaces of the participating reactants. On the other hand, in the stirred tank reactors, the contacts among the three phases depend very much on the degree of mixing. Thus, practically the interfacial boundary area among the three phases could not be controlled in the stirred tank reactors. The control on the contact boundaries among the three phases by using our new method was found to be effective to achieve reproducible conditions of reactions to get reproducible data. Furthermore, because the interfacial surface area in the packed bed reactor could be predicted, the intrinsic chemical reaction rate constants could be determined. 


\subsection{Significance}

The key point, which is also the most original point of this dissertation, is the determination of the intrinsic chemical reaction rate constants of $\mathrm{NGH}$ formation reactions, particularly $\mathrm{MGH} / \mathrm{PGH}$ formation reactions. In order to understand the comprehensive overall reaction kinetics including various mass transfer mechanisms of $\mathrm{NGH}$ formations, the data of reliable intrinsic chemical reaction rate constants are among the most important data of NGH. The data are important in the design of the equipments of some possible applications of NGH. As an illustration, below we cite some possible applications of NGH that have been discussed in various literatures and journals by other researchers. To get full benefits of all these possible applications, we need full understanding of the kinetics of $\mathrm{NGH}$ formation reactions, including the data of the intrinsic chemical reaction rate constants of the NGH formations.

During the last $20^{\text {th }}$ century, the percentage of the use of oil and NG in the world energy balance has increased from 3\% to 63\% worldwide (Makogon, 1997). It is predicted that NG will play more and more important role in the world energy balance in the future decades. Natural resources of $\mathrm{NG}$ in $\mathrm{NGH}$ state are proved to be very prospective, in terms of their abundant availability. It was found that the natural resources of NG in NGH state were by two orders of magnitude greater than the world's explored NG reserves. It makes NGH a potential future energy resource.

There are also some other possible applications of NGH. Some researchers observed that the compositions of the NG released from the NGH dissociation were different from the compositions of the original mixture of $\mathrm{NG}$ that formed the $\mathrm{NGH}$ (Englezos, 1993). It means the composition of NG mixture in the gas phase is different 
from its equilibrium composition in NGH phase. As an illustration, Table 1.1 shows some data of NGH compositions in equilibrium with NG compositions in gas phase at various equilibrium decomposition temperatures and pressures. This fact shows possible application of NGH in NG purification technology.

TABLE 1.1

NGH Equilibrium Compositions ( $\%$ by volume) ${ }^{*}$

\begin{tabular}{|c|c|c|c|c|c|c|c|}
\hline & $\mathrm{NG}$ & $P(\mathrm{MPa})$ & 0.705 & 1.17 & 6 & 10 & 20 \\
\hline Component & $\begin{array}{l}\text { Composition, } \\
\% \text { by volume }\end{array}$ & $T\left({ }^{\circ} \mathrm{C}\right)$ & 2 & 6 & 18.3 & 21 & 23.7 \\
\hline $\mathrm{CH}_{4}$ & 80 & & 49.664 & 53.782 & 63.393 & 66.377 & 70.665 \\
\hline $\mathrm{C}_{2} \mathrm{H}_{6}$ & 10 & & 2.756 & 2.734 & 3.286 & 3.817 & 4.393 \\
\hline $\mathrm{C}_{3} \mathrm{H}_{8}$ & 5 & & 34.002 & 31.174 & 25.007 & 23.122 & 19.984 \\
\hline$i-\mathrm{C}_{4} \mathrm{H}_{10}$ & 1 & & 9.839 & 8.856 & 5.849 & 4.652 & 3.390 \\
\hline$n-\mathrm{C}_{4} \mathrm{H}_{10}$ & 2 & & 3.079 & 2.751 & 1.715 & 1.302 & 0.876 \\
\hline $\mathrm{CO}_{2}$ & 2 & & 0.661 & 0.703 & 0.749 & 0.731 & 0.692 \\
\hline
\end{tabular}

*) (Makogon, 1997)

Another possible application is the application of NGH in gas storage and transportation, due to NGH specific volume that is equal to highly compressed NG. As an illustration, density of MGH at $273 \mathrm{~K}$ and $2.56 \mathrm{MPa}$ is $0.910 \mathrm{~g} / \mathrm{cm}^{3}$ (Makogon, 1997). Let the amount of water and methane in $1 \mathrm{~cm}^{3}$ of MGH be $b_{1} \mathrm{~g}$ and $b_{2} \mathrm{~g}$ respectively. Hence,

$$
b_{1}+b_{2}=0.910
$$


Furthermore, let the $\mathrm{MGH}$ have an ideal composition, i.e. $\mathrm{CH}_{4} .5 .75 \mathrm{H}_{2} \mathrm{O}$. It means,

$$
\begin{aligned}
& \frac{b_{2}}{b_{1}+b_{2}}=\frac{b_{2}}{0.910}=\frac{16.04}{16.04+(5.75)(18.016)}=0.1341 \\
& b_{2}=0.122 \mathrm{~g}
\end{aligned}
$$

with 16.04 and 18.016 are the molecular weights of methane and water respectively. Hence, in MGH phase at $273 \mathrm{~K}, 0.122 \mathrm{~g}$ of methane occupy $1 \mathrm{~cm}^{3}$ of total space at pressure as low as $2.56 \mathrm{MPa}$ only. In fact, the total space is not solely occupied by methane, but also by water. Let us compare this pressure with the pressure of $0.122 \mathrm{~g}, 1$ $\mathrm{cm}^{3}$ of ideal methane gas at $273 \mathrm{~K}$.

$$
\begin{aligned}
P & =\frac{(0.122 / 16.04) \mathrm{mol}(8.314)(\mathrm{J} / \mathrm{mol} \cdot \mathrm{K})(273) \mathrm{K}}{\left(10^{-6}\right) \mathrm{m}^{3}}=17.26 \times 10^{6} \mathrm{~J} / \mathrm{m}^{3} \\
& =17.26 \mathrm{MPa}
\end{aligned}
$$

Hence, to occupy $1 \mathrm{~cm}^{3}$ of space at $273 \mathrm{~K}, 0.122 \mathrm{~g}$ of methane need $17.26 \mathrm{MPa}$ compared to $2.56 \mathrm{MPa}$ in MGH phase.

To get full benefits of all these possible applications, we need full understanding of the kinetics of NGH formation reactions, including the data of the intrinsic chemical reaction rate constants of NGH formations, as we have already mentioned above. 


\section{CHAPTER 2}

\section{LITERATURE REVIEW}

\subsection{General description of NGH and their structures}

Natural gas hydrates $(\mathrm{NGH})$ are clathrate compounds that consist of host and guest. There is no real chemical reaction between the host and the guest. It means both host and guest keep their identity. There is no atom rearrangement during the formation of NGH. In NGH, the host is water molecules, and the guest is NG molecules. By hydrogen bonds, the host water molecules form a regular lattice structure containing many cavities (Jeffrey, 1984). The guest NG molecules occupy the cavities, and therefore stabilize the cavities. Without the occupation of the cavities by the guest NG molecules, the cavities are subject to attractive forces which cause them to collapse.

There are two principal limitations for the guest molecules to be able to stabilize the cavities (Jeffrey, 1984). The first limitation is that the guest molecules do not have either a single strong hydrogen-bond functional group, or a number of moderately strong hydrogen-bond groups. For example, carboxylic acids do not form hydrates because they have strong hydrogen-bond functional groups, i.e. the carboxylic groups. On the other hands, ethers, which do not have any hydrogen-bond functional groups, form hydrates. The second limitation is that the size of the guest molecules must be smaller than the size of each cavity, but can not be too small to stabilize the cavities. For example, neon and hydrogen do not form hydrates because the molecules are too small, so they can pass easily through the holes in the clathrate host structure. In the case of NGH, all species of 
NG molecules have no hydrogen-bond functional group. Hence, the only limitation is the size of the NG molecules.

There are three possible structures of NGH, i.e. cubic structure I (sI), cubic structure II (sII), and hexagonal structure $\mathrm{H}(\mathrm{sH})$ as shown in Figure 2.1. These hydrate crystal unit structures are composed of five cavities formed by hydrogen bonded water molecules. The five cavities are: (1) pentagonal dodecahedron $\left(5^{12}\right)$ that has twelve pentagonal faces; (2) tetrakaidecahedron $\left(5^{12} 6^{2}\right)$ that has twelve pentagonal and two hexagonal faces; (3) hexakaidecahedron $\left(5^{12} 6^{4}\right)$ that has twelve pentagonal faces and four hexagonal faces; (4) irregular dodecahedron $\left(4^{3} 5^{6} 6^{3}\right)$ that has three square, six pentagonal, and three hexagonal faces; and (5) icosahedron $\left(5^{12} 6^{8}\right)$ that has twelve pentagonal and eight hexagonal faces. The number of each cavity and water molecules per unit cell of hydrate crystal is shown in Figure 2.2. From the figure, we can see that each unit crystal of cubic structure sI consists of two $5^{12}$ (the smaller cavities) and six $5^{12} 6^{2}$ (the larger cavities) with total 46 water molecules. Each unit crystal of cubic structure sII is composed of sixteen $5^{12}$ (the smaller cavities) and eight $5^{12} 6^{4}$ (the larger cavities) with total 136 water molecules. For the case of hexagonal structure $\mathrm{sH}$, a unit crystal contains three $5^{12}$ (the smallest cavities), two $4^{3} 5^{6} 6^{3}$ (the medium cavities), and one $5^{12} 6^{8}$ (the largest cavity) with total 34 water molecules (Sloan, 1998a). 


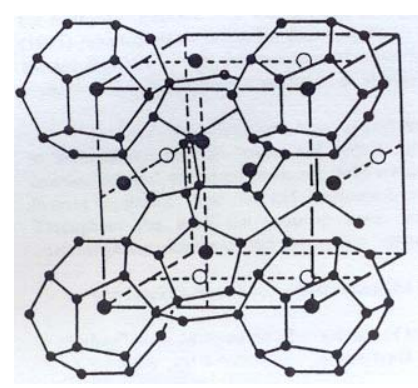

(a)

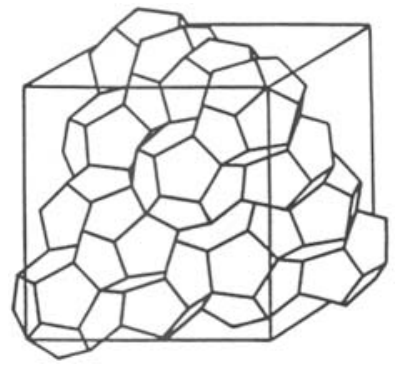

(b)

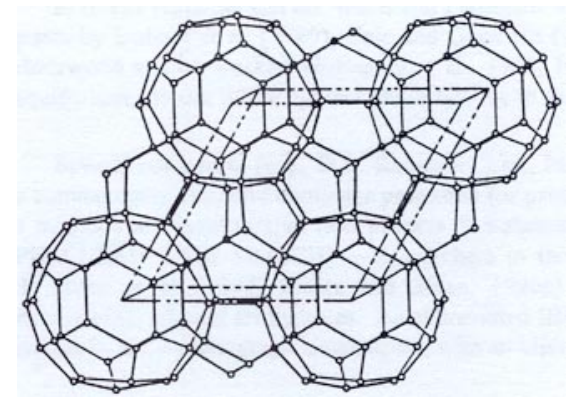

(c)

Figure 2.1. Hydrate crystal unit structures: (a) sI, (b) sII, and (c) sH (Sloan, 1998a).

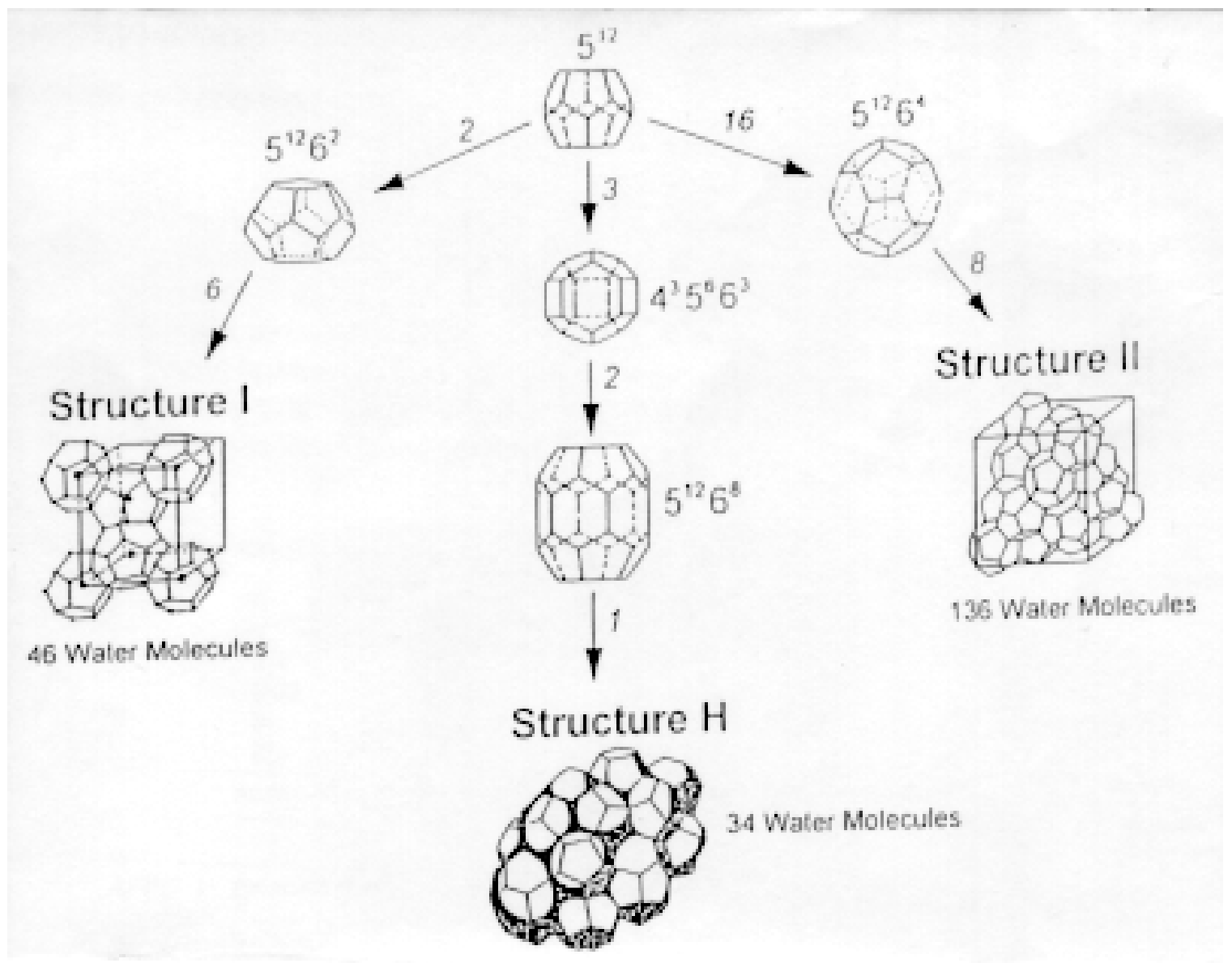

Figure 2.2. Three hydrate crystal unit structures and the five cavities (Sloan, 1998b). 
Methane forms sI hydrates. The diameter of methane molecules (i.e. $0.436 \mathrm{~nm}$ ) is sufficient to stabilize the $5^{12}$ cavities in either sI or sII or sH (cavity effective diameter $0.51 \mathrm{~nm}$ in structure sI; and $0.502 \mathrm{~nm}$ in either structure $\mathrm{sII}$ or $\mathrm{sH}$ ) with a preference for sI. The reason is that methane contributes slightly higher stability to the $5^{12} 6^{2}$ cavities (cavity effective diameter $0.586 \mathrm{~nm}$ ) in sI than $5^{12} 6^{4}$ cavities (cavity effective diameter $0.666 \mathrm{~nm}$ ) in sII, while methane is too small to stabilize $5^{12} 6^{8}$ cavity (cavity effective diameter $0.862 \mathrm{~nm}$ ) although it can stabilize $4^{3} 5^{6} 6^{3}$ cavities (cavity effective diameter $0.532 \mathrm{~nm}$ ) in $\mathrm{sH}$ (Sloan, 1998b). Hence, methane molecules occupy both the smaller $\left(5^{12}\right)$ and the larger $\left(5^{12} 6^{2}\right)$ cavities in SI, with each cavity can contain at most one methane molecule.

Propane has diameter $0.628 \mathrm{~nm}$. Its size is too large to fit into $5^{12}, 5^{12} 6^{2}$, and $4^{3} 5^{6} 6^{3}$ cavities. Propane stabilizes both $5^{12} 6^{4}$ and $5^{12} 6^{8}$ cavities with a preference for $5^{12} 6^{4}$, the smaller one between the two. As a result, propane molecules form sII hydrates and occupy only the larger cavities (Sloan, 1998b). Each larger cavity can contain at most one propane molecule.

The ratio between the number of water molecules and the number of guest molecules is known as hydration number. As we discussed above, methane can occupy both the larger and the smaller cavities of sI, with each cavity can contain at most one methane molecule. Because each unit crystal of sI consists of total 8 cavities formed by 46 water molecules, the ideal hydration number of $\mathrm{MGH}$ is equal to $(46 / 8)=5.75$. In case of PGH, propane can occupy the larger cavities of sII only, with each larger cavity can contain at most one propane molecule. Because each unit crystal of sII contains totally 8 larger cavities and 136 water molecules, the ideal hydration number of PGH is equal to 
$(136 / 8)=17$. The real hydration numbers are always bigger than the ideal hydration numbers, because it is impossible for the guest molecules to occupy all cavities (Sloan, 1998a). The variation in hydration numbers, different from their ideal values, causes hydrates to be called non-stoichiometric compounds.

\subsection{Kinetics of hydrate formation}

When dealing with hydrate formation kinetics, it is usual to divide the hydrate formation process into two separate periods (Skovborg et al., 1993). The first period involves the formation of hydrate nuclei. The period is often called the induction period. During the induction period, small hydrate crystals (nuclei) are formed. Then, the hydrate nuclei grow to achieve their critical size. Before the nuclei reach their critical size, they are not stable and some are dissolved back to the solution. Above their critical size, the nuclei are stable and will continuously grow (Sloan, 1998a). The induction time is basically the time needed for the nuclei to achieve their critical size for continuous growth. Previous studies showed that induction time can not be acceptably predicted due to the stochastic nature of the nucleation phenomenon. Fortunately, the induction time could be eliminated from the reacting system, if enough hydrate seeds with sizes above critical size are available in the reacting system since the start of the reaction. Some previous studies showed that in dissociation, hydrates did not totally decompose but preserved a crystalline-hydrate structure (Makogon, 1981). The partial structures acted as hydrate nuclei and enabled hydrates to form more readily in the reacting system.

We should mention here that our research did not include any investigations on the induction period of $\mathrm{MGH} / \mathrm{PGH}$ formation. Our goal is to determine the intrinsic 
chemical reaction rate constants of $\mathrm{MGH} / \mathrm{PGH}$ formations during the growth period (the second period that we will discuss later). To eliminate the induction period from our experiments, we formed MGH/PGH seeds, and then maintained the seeds in the reacting systems from run to run of the experiments by maintaining temperature of the system at the temperature of reaction, i.e. $273.5 \mathrm{~K}$, and did the $\mathrm{MGH} / \mathrm{PGH}$ formation reactions of the next runs in an hour after the dissociation experiments finished.

The second period is known as the growth period. The growth period begins when hydrate seeds have reached their critical size. During the growth period, hydrate crystals will only grow. In contrast to the induction period, the growth period is predictable. Hydrate growth has been the object of some researches. We will discuss some of the researches on the kinetics of NGH growth, including the model proposed by each researcher. We should explain here that the citations of the models proposed by other researchers are mainly for the purpose of comparison to our model, not the bases of the derivation of our model. Our model was derived based on our reacting system (a special packed bed reactor), which was different from the other researchers' reacting system (semi batch stirred tank reactors). Although at the same temperature of reaction, the intrinsic chemical reaction rate constant of NGH formation must be the same in all kinds of reacting systems, but the model to get the value of the intrinsic chemical reaction rate constant could be different from one reacting system to other reacting systems.

Vysniauskas and Bishnoi (1983) reported kinetics data of MGH formation. They did their experiments in a semi batch stirred tank reactor at isothermal and isobaric conditions. Temperature of reactions was varied in the range of 274 to $284 \mathrm{~K}$; while pressure ranged from 3 to $10 \mathrm{MPa}$. The MGH growth kinetics was dependent on the gas- 
liquid interfacial area, pressure, temperature, and degree of super-cooling, but was not dependent on the history of water. The MGH formation rate increased with increasing gas-liquid interfacial area. The increase of gas-liquid interfacial area was achieved by increasing stirring rate. An increase in pressure will cause the increase of the MGH formation rate; while an increase in temperature will decrease the MGH formation rate. The degree of super-cooling was defined as the difference between the MGH equilibrium temperature at the experimental pressure and the experimental temperature. The degree of super-cooling gave positive effects on the MGH formation rate, i.e. the increase of supercooling degree increased the MGH formation rate. The history of water affected the induction time, but it had no effects on the kinetics of the MGH formation in the growth period. For example, the induction time was reduced to zero when the water was re-used immediately after MGH dissociation; but the mean reaction rates in the growth period stayed the same. Furthermore, a semi empirical model was proposed to correlate the data. The model was represented by Equation (2.1).

$$
r=A a_{s} \exp \left(-\frac{\Delta E_{a}}{R T}\right) \exp \left(-\frac{a}{\Delta T^{b}}\right) P^{\gamma}
$$

with $r$ is the consumption rate of methane; $A$ is a lumped pre-exponential constant; $a_{s}$ is the effective surface area of gas-water interface; $\Delta E_{a}$ is the apparent energy activation of MGH formation; $R$ is gas constant; $T$ is temperature of reaction; $\Delta T$ is degree of supercooling; $P$ is system pressure; $a$ and $b$ are two empirical parameters; and $\gamma$ is the overall order of the reaction. $A, \Delta E_{a}, a, b$, and $\gamma$ were determined by least square fit of the experimental data. The results were $A=4.554 \times 10^{-26} \mathrm{~cm}^{3} /\left(\mathrm{cm}^{2} \cdot \min \cdot b_{a r}{ }^{\gamma}\right) ; \Delta E_{a}=-106.204$ $\mathrm{kJ} / \mathrm{mol} ; \gamma=2.986 ; a=0.0778 \mathrm{~K}^{b}$; and $b=2.411$. 
The same model was used to correlate kinetics data of EGH formation (Vysniauskas and Bishnoi, 1985). The data were obtained from experiments using a semi batch stirred tank reactor that was operated at isothermal and isobaric conditions at temperature range 274 to $282 \mathrm{~K}$, and pressure range 0.6 to $2.6 \mathrm{MPa}$. The kinetics was a function of gas-liquid interfacial area, pressure, temperature, and degree of super-cooling, as shown by the model equation. Due to the lack of data, the values of $a$ and $b$ were assumed to be the same as the one obtained in MGH experiments, i.e. $a=0.0778 \mathrm{~K}^{b}$; and $b=2.411$. Furthermore, least square fit on the data gave values of $A=2.400 \times 10^{-29}$ $\mathrm{cm}^{3} /\left(\mathrm{cm}^{2} \cdot \min \cdot \mathrm{bar}^{\gamma}\right) ; \Delta E_{a}=-133.015 \mathrm{~kJ} / \mathrm{mol} ;$ and $\gamma=2.804$

Englezos et al. (1987a) proposed a kinetic model of the MGH and EGH formations based on crystallization theory ${ }^{1}$. They included mass transfer effects in their model. The interfacial mass transfer model was based on the film theory. The growth of the MGH/EGH crystals is following two steps: (1) diffusion of the dissolved gas from the bulk of the solution to the crystal-liquid interface through liquid film around the crystals; and (2) adsorption of the dissolved gas on the surface of the crystals, which includes stabilization of the water structure. The second step is the intrinsic reaction of $\mathrm{MGH} / \mathrm{EGH}$ formation. These two steps of MGH/EGH formation are represented by Equation (2.2).

$$
\left(\frac{d n}{d t}\right)_{p}=K * A_{p}\left(f-f_{e q}\right)
$$

where

$$
\frac{1}{K^{*}}=\frac{1}{k_{r}}+\frac{1}{k_{d}}
$$

\footnotetext{
${ }^{1}$ See Appendix B for the more complete derivation of the Englezos et al.'s model.
} 
Here, $\left(\frac{d n}{d t}\right)_{p}$ is the rate of methane/ethane consumption into $\mathrm{MGH} / \mathrm{EGH} ; K^{*}$ is the combined rate parameter; $A_{p}$ is the surface area of MGH/EGH crystal; $f$ is fugacity of the dissolved methane/ethane at pressure and temperature of experiment; $f_{e q}$ is fugacity of the dissolved methane/ethane at three-phase equilibrium pressure and experimental temperature; $k_{r}$ is the intrinsic reaction rate constant of $\mathrm{MGH} / \mathrm{EGH}$ formation; and $k_{d}$ is the mass transfer coefficient of the dissolved methane/ethane around the MGH/EGH crystals. Different from the Vysniauskas and Bishnoi's model, the driving force in the Englezos et al.'s model is the deviation from the equilibrium condition, instead of the experimental pressure. We can also see that according to the model, the $\mathrm{MGH} / \mathrm{EGH}$ growth took place throughout the bulk of the liquid phase, without limitation to the gasliquid interface. The model was applied on the experimental data of $\mathrm{MGH} / \mathrm{EGH}$ formation obtained from experiments using an isothermal-isobaric-semi-batch-stirredtank-reactor. The experiments were done at four temperatures, i.e. 274, 276, 279, and 282 $\mathrm{K}$, pressure range from 3.29 to 8.90 $\mathrm{MPa}$ for $\mathrm{MGH}$ and 0.64 to $2.19 \mathrm{MPa}$ for $\mathrm{EGH}$, and stirring rates from 300 to $450 \mathrm{rpm}$. Analysis on the data showed: (1) the greater the deviation of $f$ from $f_{\text {eq }}$, the faster the MGH/EGH formation rate; (2) very weak dependence of $K^{*}$ on temperature $(T)$, as shown by insignificant increase in $K^{*}$ by increasing $T$; (3) experiments were performed in the kinetic regime, so $K^{*}$ was essentially the intrinsic reaction rate constant of the MGH/EGH formation; and (4) at below $400 \mathrm{rpm}$ stirring rate, $K^{*}$ was affected by stirring rate; while at $400 \mathrm{rpm}$ or greater stirring rate, the effects of stirring rate on $K^{*}$ was no longer observed.

Englezos et al. (1987b) extended the model for the kinetics of NGH formation from mixtures of methane and ethane. The experiments were done in the same reactor 
used in the pure methane and ethane experiments. The reaction took place at temperatures from 273 to $284 \mathrm{~K}$, pressures from 0.68 to $5.60 \mathrm{MPa}$, and stirring rate $400 \mathrm{rpm}$. The pressure was chosen such that no condensation of the gas occurred during the experiments. The results showed that the NGH formation rate was proportional to a linear combination of the driving force of each pure NG, as shown by Equation (2.4).

$$
\left(\frac{d n}{d t}\right)_{p}=\sum_{1}^{2}\left(\frac{d n_{j}}{d t}\right)_{p}=\sum_{1}^{2} K_{j}^{*} A_{p}\left(f-f_{e q}\right)_{j}
$$

The kinetics parameters were those obtained from the pure NGH experiments. The effect of the composition of the gas mixture was taken into account indirectly through the $f$ and $f_{e q}$ calculations.

Dholabai et al. (1993) used the Englezos et al.'s model to study the kinetics of MGH formation in aqueous electrolyte solutions. As the electrolytes, they used $\mathrm{NaCl}$ and $\mathrm{KCl}$. The experiments were carried out in an isothermal-isobaric-semi-batch-stirred-tankreactor. The experiments were done at temperatures from 270 to $274 \mathrm{~K}$, pressure range from 3.78 to $7.08 \mathrm{MPa}$, and stirring rate $400 \mathrm{rpm}$. The kinetic parameter was that obtained from the MGH experiments in pure water. The effect of the electrolytes was taken into account indirectly through the $f_{e q}$ calculations. The presence of electrolytes in the solution caused the increase of the three phase equilibrium pressure at a given temperature. It meant $f_{e q}$ also increased. As a result, the driving force of the reaction $(f-$ $\left.f_{e q}\right)$ decreased.

Malegaonkar et al. (1997) used the Englezos et al.'s model to study the kinetics of carbon dioxide hydrate and MGH formation. The kinetics data of carbon dioxide hydrate formation were obtained from experiments in a semi-batch-stirred-tank-reactor under 
isothermal and isobaric conditions. Experiments were conducted at three different temperatures of 274,276 , and $278 \mathrm{~K}$, pressure range of 1.59 to $2.79 \mathrm{MPa}$, and stirring rate $400 \mathrm{rpm}$. They modified the Englezos et al.'s model to take into account the high solubility of carbon dioxide in water. Furthermore, they found an inconsistency in the Englezos et al.'s model ${ }^{2}$. They recalculated the combined rate parameter $K^{*}$ of MGH formation using the modified Englezos et al.'s model and got a significant improvement. As an illustration, using unmodified Englezos et al.'s model, $K^{*}$ at $274 \mathrm{~K}$ was $0.65 \times 10^{-5}$ $\mathrm{mol} / \mathrm{m}^{2} \cdot \mathrm{s} \cdot \mathrm{MPa}$ (Englezos et al., 1987a). After Malegaonkar et al.'s modification, $K^{*}$ at $274 \mathrm{~K}$ is $3.1 \times 10^{-5} \mathrm{~mol} / \mathrm{m}^{2} \cdot \mathrm{s} \cdot \mathrm{MPa}$.

Skovborg and Rasmussen (1994) analyzed the Englezos et al.'s model and their experimental data. They pointed out some points of the model.

(1) The Englezos et al.'s model predicts the increasing rate with increasing time. The reason is the NGH formation rate is proportional to the surface area of the NGH crystals according to the model. The surface area increases with time as the NGH crystals grow. In contrast to the model prediction, the experimental data showed decreasing rate of NGH formation at higher time. The possible explanation for the decreasing rate of NGH formation with time is the fact that the NGH crystals blocked the gas-water contact, and in effect decreased the gas-water interfacial area. This effect is not counted in the model.

(2) The experimental data showed a constant NGH growth rate as a function of time with a decreasing tendency at longer times. A possible explanation for this fact is that the reaction rate did not depend on the total area of NGH crystals, and the reaction was controlled by mass transfer of the dissolved NG.

\footnotetext{
${ }^{2}$ See Appendix B for the modification of Englezos et al.'s model for NGH formation.
} 
(3) If we assign the value of $K^{*}$ of MGH formation too high (for example $K^{*}=$ $0.65 \times 10^{-5} \mathrm{~mol} / \mathrm{m}^{2} \cdot \mathrm{s} \cdot \mathrm{MPa}$ ) or too low (for example $K^{*}=0.65 \times 10^{-9} \mathrm{~mol} / \mathrm{m}^{2} \cdot \mathrm{s} \cdot \mathrm{MPa}$ ), the rate of MGH growth increases with time. At intermediate value of $K^{*}$ (for example $K^{*}=$ $\left.0.65 \times 10^{-7} \mathrm{~mol} / \mathrm{m}^{2} \cdot \mathrm{s} \cdot \mathrm{MPa}\right)$, the $\mathrm{MGH}$ growth rate is constant with time. It is in accordance with the constant MGH growth rate of the experimental data. Unfortunately, the reduction of $K^{*}$ from $0.65 \times 10^{-5} \mathrm{~mol} / \mathrm{m}^{2} \cdot \mathrm{s} \cdot \mathrm{MPa}$ to $0.65 \times 10^{-7} \mathrm{~mol} / \mathrm{m}^{2} \cdot \mathrm{s} \cdot \mathrm{MPa}$ causes poor agreement between the model and the experimental data. A possible explanation for this fact could be mass transfer coefficient $k_{L}$ of the dissolved methane was assigned wrong values. Data of $k_{L}$ was obtained experimentally in a condition where no MGH was formed. It is possible that the existence of MGH in the system changes the hydrodynamic of the system, which then affects the value of $k_{L}$.

(4) Experimental data showed the effect of the stirring rate on $K^{*}$ of MGH formation. The decrease of stirring rate from 400 to $300 \mathrm{rpm}$ caused the decrease of $K^{*}$ with a factor of approximately 300. The mass transfer coefficient of the dissolved methane around the MGH crystal seeds of critical size $40 \mathrm{~nm}$ at $274 \mathrm{~K}$ is approximately $1.0 \mathrm{~mol} / \mathrm{m}^{2} \cdot \mathrm{s} \cdot \mathrm{MPa}$. This value is much larger than the value of $K^{*}$ of MGH formation at $274 \mathrm{~K}$ and $400 \mathrm{rpm}$, which is $0.65 \times 10^{-5} \mathrm{~mol} / \mathrm{m}^{2} \cdot \mathrm{s} \cdot \mathrm{MPa}$. Hence, MGH formation rate must not be controlled by the mass transfer rate of dissolved methane around the MGH crystals. Because the intrinsic reaction rate should not be affected by stirring rate, it means $K^{*}$ did not control the overall methane consumption rate. The change in stirring rate will affect the mass transfer of dissolved methane from gas-water interface to the bulk of the liquid, due the change in both gas-water interfacial area and mass transfer coefficient of the dissolved methane. 
(5) Englezos et al. (1987a) set the value of the nucleation rate constant ${ }^{3}$ very low, i.e. $10^{-3}$, which implied that there was no secondary nucleation. Because at initial condition, all NGH crystals had the same size, which was equal to their critical size, it meant all NGH particles had the same size at any time and grew with the same rate. As a consequence, the population balance could be replaced by a simple relation between the total surface area of the NGH crystals and the amount of NG consumed ${ }^{4}$.

(6) The Englezos et al.'s model is very sensitive to the number of moles of NG consumed at the turbidity point ${ }^{5}$. In some cases, such as when no induction time was observed due to the almost spontaneous formation of critical size NGH seeds, the moles of NG consumed at the turbidity point could not be obtained experimentally.

Based on the above analysis, Skovborg and Rasmussen (1994) ${ }^{6}$ proposed a simplified model of Englezos et al.'s model, based on the assumption that the transport of NG molecules from the gas-water (1) interface to the bulk of the liquid water phase is the rate determining step in the overall NGH formation process. The Skovborg and Rasmussen's model is shown by Equation (2.5).

$$
\frac{d n}{d t}=k_{L} A(g-l) c_{w 0}\left(x_{i n t}-x_{b}\right)
$$

where $k_{L}$ is the mass transfer coefficient of the dissolved NG in the water liquid film; $A_{(g \text { - }}$ l) is gas-liquid interfacial area; $c_{w 0}$ is the initial concentration of water molecules; $x_{\text {int }}$ is the mole fraction of the dissolved NG in water (1) phase at the gas-liquid interface in equilibrium with the gas phase at system pressure and temperature; and $x_{b}$ is the mole

\footnotetext{
${ }^{3}$ Read Appendix B, sub chapter B.4. Estimation of $\mu_{2}(t)$.

${ }^{4}$ Read Equation B.39 in Appendix B. Here, $N_{p}$ remained constant, so we have the relation between NGH crystal size $(r)$ and the amount of NG consumed $(n) . r$ replaces $r_{c r}$, and $n$ replaces $n_{t b}$ in the equation.

${ }^{5}$ The turbidity point is the time when turbidity starts to appear in the liquid phase. The turbidity in the liquid phase is caused by sudden appearance of NGH particles. See Appendix B, sub chapter B.6. Determination of model parameters.

${ }^{6}$ Read Appendix C for more discussion on Skovborg and Rasmussen's model.
} 
fraction of dissolved NG in the bulk water liquid phase in equilibrium with the NGH phase at system pressure and temperature. Furthermore, the model was used to evaluate the data of MGH and EGH experiments done in a semi-batch-stirred-tank-reactor at the same temperature and pressure as the Englezos et al.'s experiments. The model parameter to be determined was $\mathrm{k}_{\mathrm{L}}$.

The Skovborg and Rasmussen's model of course could not predict the intrinsic reaction rate constant of NGH formation. The fact that the intrinsic reaction rate constants of NGH formation might not have been successfully determined, encouraged us to do this research. To have a reaction condition where mass transfer resistance of dissolved gas could be minimized, we used a special packed bed reactor in the experiments. The bed of the reactor consisted of uniform-sized-spherical-glass-beads, with water was distributed homogeneously at the points of contacts among the glass beads. The system provided tiny units of reactors in form of water bridges of size in the order of liquid film thickness. Hence, the mass transfer resistance in the bulk of liquid existed in the Englezos et al.'s and Skovborg and Rasmussen's experiments could be eliminated in our reacting system. It left us with only two resistances: (1) the mass transfer resistance of dissolved NG in the liquid film between the gas-water interface and water-NGH crystal interface; and (2) the intrinsic reaction rate resistance.

Kono et al. (2002) used the packed bed reactor to study the kinetics of MGH formation and decomposition. The MGH formation reaction was done at a pressure range 6.8 to $13.6 \mathrm{MPa}$, and temperature $273.5 \mathrm{~K}$. Because the reactor was a batch reactor, the pressure inside the reactor decreased with time due to methane consumption into MGH. The reactor pressure data were converted to data of moles of methane in the gas phase as 
a function time. The data were used to determine the rate constant $\left(k_{f}\right)$ and the overall $\operatorname{order}\left(n^{*}\right)$ of the MGH formation reaction, following Equation (2.6).

$$
-\frac{d n_{C H_{4}}}{d t}=k_{f} n_{C H_{4}}^{n^{*}}
$$

In this research, a new physical model that suits the reacting system is proposed. The model is used to determine the intrinsic reaction rate constants of $\mathrm{MGH}$ and $\mathrm{PGH}$ formation reactions. 


\section{CHAPTER 3}

\section{PHYSICAL MODEL AND RELEVANT EXPERIMENTAL}

\section{APPROACH}

In our special packed bed reactor, there were four phases exist during the reaction, i.e. water in liquid phase, methane or propane in gas phase, MGH or PGH in NGH-solid phase, and inert spherical glass bead particles in inert spherical glass-solid phase. Water in liquid phase was dispersed in the reactor as the water bridges at the points of contacts among the glass bead particles as shown in Figure 3.1. Each water bridge represented a tiny unit of reactor (micro-reactor). This kind of structure provided large surface area of NG (g) - water (l) interface, although there was no mechanical stirring. The larger the surface area of the NG (g) - water (1) interface, the higher the consumption rates of NG into NGH. The rate of NGH formation is proportional to the surface area of the NG $(\mathrm{g})$ water (l) interface as shown later by the physical model that suits the reaction system.

\subsection{Assumptions and the design experimental conditions}

Our research was focused on the growth rate of MGH/PGH formation. Some experiments were done to form MGH/PGH seeds in the water bridges. Then, MGH/PGH seeds were maintained in the water bridges by maintaining the temperature inside the reactor at the temperature of reaction. No induction time observed was the indication that MGH/PGH seeds were not destroyed after MGH/PGH dissociation. Experimental results 
showed that after a couple runs by maintaining the temperature inside the reactor at the temperature of reaction, there was no induction time observed.

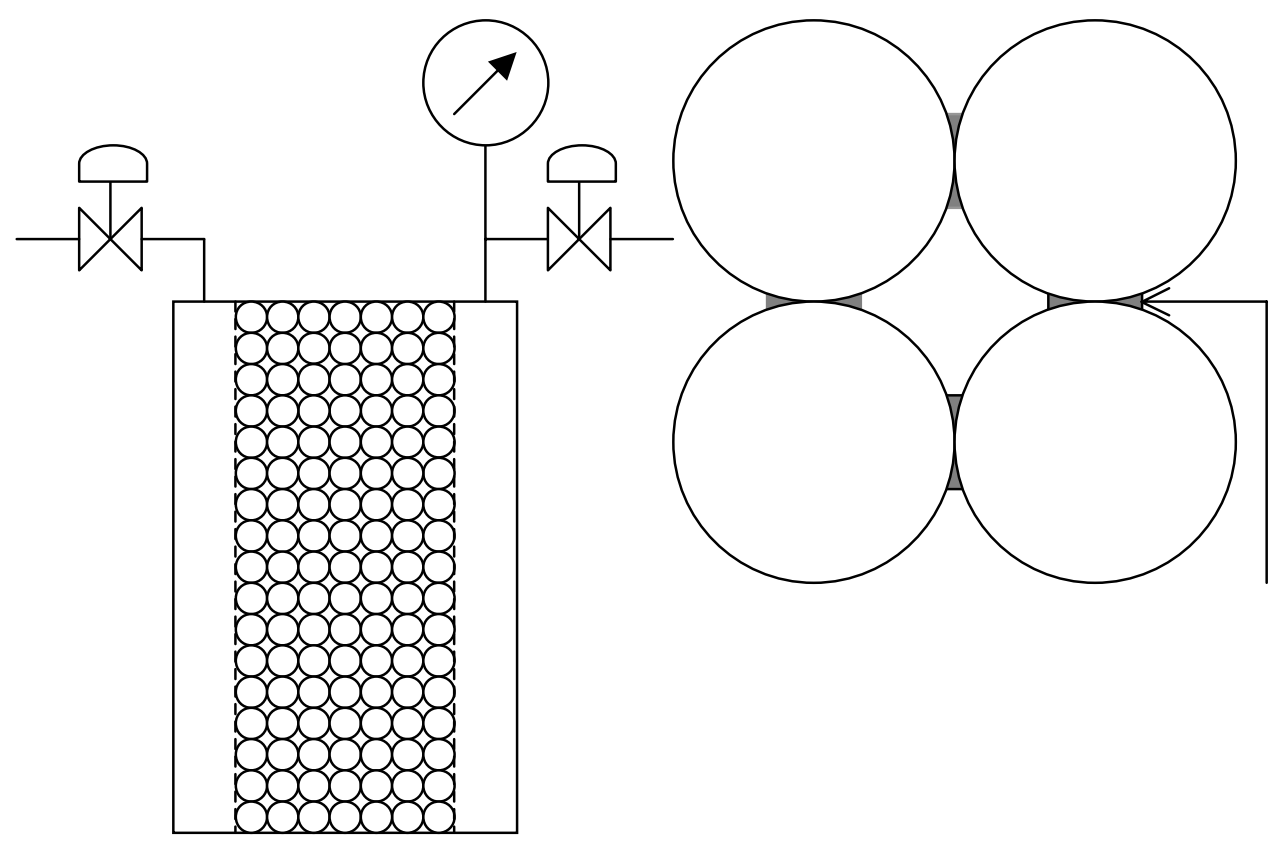

Water Bridge

Figure 3.1. Schematic pictures of the special packed bed reactor with glass beads as the packed bed and water bridges held among the spherical glass beads.

Several assumptions and designed experimental conditions of the reacting system that are the bases of the physical model of $\mathrm{MGH} / \mathrm{PGH}$ formation kinetics are as follows.

1. Our system was not agitated, so $\mathrm{MGH} / \mathrm{PGH}$ formation occurred in the liquid film at the gas-water (1) interface where the concentration of the dissolved methane/propane was the highest.

2. Before $\mathrm{MGH} / \mathrm{PGH}$ was formed, methane/propane dissolved into water (1) first. Then, $\mathrm{MGH} / \mathrm{PGH}$ formation reaction took place on the surface of $\mathrm{MGH} / \mathrm{PGH}$ 
seeds during the growth period. This step created mass transfer resistance in water (1) phase as shown by a thin stagnant liquid film between the surface of MGH/PGH crystals and the gas-water (1) interface. The justification of a single liquid film will be discussed later in the derivation of the physical model of MGH/PGH formation.

3. Water (1) was homogeneously dispersed as water bridges among the spherical glass beads particles in our packed bed reactor. Hence, at each point of contact between glass bead particles, there existed the corresponding size of liquid amount as the water-bridge, as shown in Figure 3.1. Because of capillary force, water stayed in its position.

4. The size of respective single water bridge was very small. The MGH/PGH seeds were either swimming around continuously in the water bridges or attaching on the wetted surfaces of the glass beads. If the first assumption that MGH/PGH seeds were swimming around continuously was correct, then $\mathrm{MGH} / \mathrm{PGH}$ was formed either right on the gas-water (l) interface or everywhere in the water bridges. If the second assumption that MGH/PGH seeds were attaching on the wetted surface of the glass beads was correct, then MGH/PGH grew from the wetted surfaces of the glass beads. Because the surface area of the wetted surface of the glass beads was in the order of the surface area of gas-water (1) interface, then for both assumptions, we could assume that the total surface area of MGH/PGH crystals was approximately equal to the surface area of mass transfer, i.e. the surface area of the gas-water (1) interface. 
5. The size of a single water bridge was very small, so we could consider its meniscus as an arch of a circle.

6. We used high concentration of methane/propane, and the size of glass beads was selected such that the pore size of the bed was big enough. Hence, mass transfer resistance in the gas phase could be neglected. Because different sizes of glass beads will give different bed-pore-sizes, two glass bead sizes were used in the experiments to check the effect of the bed-pore-size on the reaction rate could be neglected.

7. At any instant, the rate of methane/propane consumption by the MGH/PGH was equal to the rate of methane/propane consumption from the gas phase. Hence, no rate of accumulation of dissolved methane/propane in the water liquid film.

8. Isothermal condition could be maintained during reaction. Hence, no heat transfer effects were involved.

\subsection{Model of water bridges}

Before we derived the physical model of $\mathrm{MGH} / \mathrm{PGH}$ formation that suits the reacting system, we needed to model the geometry of water bridges on the points of contacts among glass beads. We adopted the model of the distribution of water in an ideal soil, which was defined as a collection of spheres all having the same radius, packed together in a systematic way, and free from any colloidal material (Keen, 1924). We should explain here that the ideal soil model was an idealization of the real condition in our packed bed reactor, because the homogeneity of the glass bead size in our packed bed 
reactor was not always guaranteed. The schematic picture of a single water bridge was then shown in Figure 3.2.

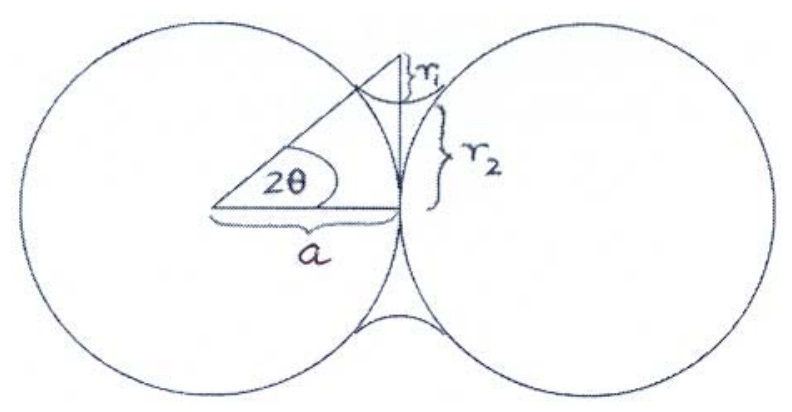

Figure 3.2. Model of a single water bridge.

Based on the model of a single water bridge, we could derive the equations for the radius of the meniscus of the water bridge $\left(r_{1}\right)$, the shortest distance between the contact point of the two glass beads and the meniscus of the water bridge $\left(r_{2}\right)$, the surface area ( $\left.A_{\text {single }}\right)$ and the volume $\left(V_{\text {single }}\right)$ of the water bridge, as shown by Equations (3.1) to (3.4) ${ }^{1}$.

$$
\begin{aligned}
& r_{1}=a \frac{(1-\cos 2 \theta)}{\cos 2 \theta} \\
& r_{2}=a \frac{(\sin 2 \theta+\cos 2 \theta-1)}{\cos 2 \theta} \\
& A_{\text {sin gle }}=\frac{8 \pi a^{2} \sin ^{2} \theta}{\cos 2 \theta}\left\{\tan 2 \theta\left(\frac{\pi}{2}-2 \theta\right)-2 \sin ^{2} \theta\right\} \\
& V_{\text {sin gle }}=\frac{8 \pi a^{3} \sin ^{4} \theta}{\cos ^{2} 2 \theta}\left\{1-\tan 2 \theta\left(\frac{\pi}{2}-2 \theta\right)\right\}
\end{aligned}
$$

where $a$ is the radius of glass bead particles; and $\theta$ is the angle as defined in Figure 3.2.

\footnotetext{
${ }^{1}$ See Appendix A for the derivations of Equation (3.1) to (3.4).
} 
To get the total surface area of gas-water (l) interface, we needed to approximate the total number of water bridges inside the bed $(N)$ as follows. Let $N_{g b}$ be the number of glass bead particles in the bed, $W$ be the total weight of the glass beads in the bed, and $\rho_{s}$ be the true density of the glass beads. Then, $N_{g b}$ is equal to the total weight of the glass beads divided by the weight of a single glass bead as shown by Equation (3.5).

$$
N_{g b}=\frac{3 W}{4 \pi a^{3} \rho_{s}}
$$

Because the number of contacts of each glass bead is the coordination number $C$, the total number of contacts among glass beads in the bed is equal to $N_{g b} C$ with each contact is counted twice. Hence, the total number of water bridges inside the bed $N$, which is equal to the total number of contacts among glass beads, is equal to $N_{g b} C / 2$ as shown in Equation (3.6).

$$
N=\frac{3 C W}{8 \pi a^{3} \rho_{S}}
$$

Furthermore, the total surface area of gas-water (1) interface, $A$, is approximately equal to the surface area of a single water bridge times the total number of water bridges. Using Equations (3.3) and (3.6), we get Equation (3.7) for $A$.

$$
A=\frac{3 C W}{a \rho_{S}} \frac{\sin ^{2} \theta}{\cos 2 \theta}\left\{\tan 2 \theta\left(\frac{\pi}{2}-2 \theta\right)-2 \sin ^{2} \theta\right\}
$$

From Equation (3.7), we know that to get the value of $A$, we need the data of the coordination number $(C)$, the total weight of glass beads in the bed $(W)$, glass bead true density $\left(\rho_{s}\right)$, and $\theta$ as defined in Figure 3.2.

$W$ and $\rho_{s}$ were measured by experiments. Data of bulk density $\left(\rho_{b}\right)$ and $\rho_{s}$ for the two sizes of glass beads used in the experiments are shown in Table 3.1. Here, the bulk 
density is defined as the ratio of the total weight of glass beads to the total volume occupied by the glass beads. The true density is then defined as the ratio between the weight of single glass bead and its volume.

\section{TABLE $3.1^{2}$}

Glass Bead Bulk Density $\left(\rho_{b}\right)$ and True Density $\left(\rho_{s}\right)$, and the Corresponding Porosity of the Bed $(\varepsilon)$ and Coordination Number $(C)$

\begin{tabular}{|c|c|c|c|c|}
\hline$\left.d_{p}, \mu \mathrm{m}^{*}\right)$ & $\rho_{b}, \mathrm{~g} / \mathrm{mL}$ & $\rho_{s}, \mathrm{~g} / \mathrm{mL}$ & $\varepsilon$ & $\left.C^{* *}\right)$ \\
\hline 240 & 1.5085 & 2.4694 & 0.3891 & 8 \\
\hline 510 & 1.5410 & 2.4799 & 0.3786 & 8 \\
\hline
\end{tabular}

*) $d_{p}$ is the diameter of glass beads

**) (Haughey and Beveridge, 1969). Please notice that $C=8$ is corresponding to $\varepsilon=$ 0.3954; while $C=10$ is corresponding to $\varepsilon=0.3019$. Our data of $\varepsilon$ are closer to $\varepsilon=$ 0.3954 than to $\varepsilon=0.3019$. The values of $\varepsilon$ that are smaller than 0.3954 indicate that there was inhomogeneity of the size of the glass beads used in the experiments. As an idealization, we assumed that the coordination number of the bed could be represented by $C=8$.

There is correlation between coordination number $(C)$ and porosity of the bed $(\varepsilon)$. Porosity of the bed $(\varepsilon)$ is defined as the ratio of the volume that is not occupied by glass beads in the bed to total volume of the bed. Using data of $\rho_{b}$ and $\rho_{s}$, we can calculate $\varepsilon$ using Equation (3.8).

\footnotetext{
${ }^{2}$ See Appendix D for the determination of $\rho_{\mathrm{b}}$ and $\rho_{\mathrm{s}}$.
} 


$$
\begin{aligned}
\varepsilon & =\frac{\text { volume not occupied by glass beads }}{\text { total volume }} \\
& =\frac{(\text { total volume })-(\text { volume of glass beads })}{\text { total volume }} \\
& =1-\frac{\text { volume of glass beads }}{\text { total volume }}=1-\frac{\left(\mathrm{W} / \rho_{S}\right)}{\left(\mathrm{W} / \rho_{b}\right)} \\
\varepsilon & =1-\frac{\rho_{b}}{\rho_{S}}
\end{aligned}
$$

Datum of $\varepsilon$ for each glass bead size is presented in Table 3.1. The coordination number (C) for the corresponding $\varepsilon$ can be found in reference (Haughey and Beveridge, 1969). The data of $C$ are also presented in Table 3.1. We should explain here that the values of $C$ showed in Table 3.1 are idealization of the actual value of $C$ of the packed bed.

To predict $\theta$, we needed to measure the moisture content of the bed $(M)$ which is defined as the ratio of water weight to the glass bead weight in the bed. Obviously, by adding a known amount of water to a known amount of glass beads, we can calculate $M$. Furthermore, what we needed was a relation between $M$ and $\theta$. Hence, for a certain $M$, we can calculate $\theta$. The relation between $M$ and $\theta$ can be derived as follows. By definition,

$$
M=\frac{\text { water weight }}{\text { glass bead weight }}=\frac{N V_{\text {sin gle }} \rho_{w}}{W}
$$

where $\rho_{w}$ is the density of water (1). By substitution of Equations (3.4) and (3.6) into Equation (3.9), we get Equation (3.10).

$$
M=\frac{3 C \rho_{w}}{\rho_{s}} \frac{\sin ^{4} \theta}{\cos ^{2} 2 \theta}\left\{1-\tan 2 \theta\left(\frac{\pi}{2}-2 \theta\right)\right\}
$$

We defined one more parameter, i.e. relative saturation of packed bed void by water $(S)$. The relative saturation of packed bed void by water $(S)$ is the ratio of the 
volume of the water to the volume that is not occupied by glass beads. The equation for $S$ can be derived as follows.

$$
\begin{aligned}
S & =\frac{\text { water volume }}{\text { volume of the space of the bed that is not occupied by glass beads }} \\
& =\frac{\text { water volume }}{(\text { total volume })-(\text { volume of glass beads })} \\
& =\frac{\left\{\frac{\text { water volume }}{\text { volume of glass beads }\}}\right.}{\left\{\frac{\text { total volume }}{\text { volume of glass beads }\}}-1\right.} \\
& \left.=\frac{(\text { water weight }) / \rho_{w}}{(\text { glass bead weight }) / \rho_{S}}\right\} \\
S & =\frac{(1-\varepsilon) M \rho_{S}}{\varepsilon \rho_{w}}\{-1
\end{aligned}
$$

In the experiments, it was important to provide water as much as possible so that the pressure drop due to NG consumption into NGH can be observed. The other reason was the fact that water-glass beads mixing to get homogeneously distribution of water in the bed was easier to do for larger $S$. To determine how much $S$ should be used in the experiments, we did the following calculation. From Table 3.1, we knew that the coordination number of our packed bed was approximately 8 . It meant approximately, our packed bed had an orthorhombic structure as shown schematically in Figure 3.3. Unit layers of the three different sides of view of the packing structure are shown in Figure 3.4. Unit layer of the top/bottom side of view is shown in Figure 3.4 (a). If the plane of 
the left/right side of view is rotated as far as $30^{\circ}$, we will see the same unit layer as the one of the top/bottom side of view. Figure 3.4 (b) shows the front/back side of view.

Top view

Right side view

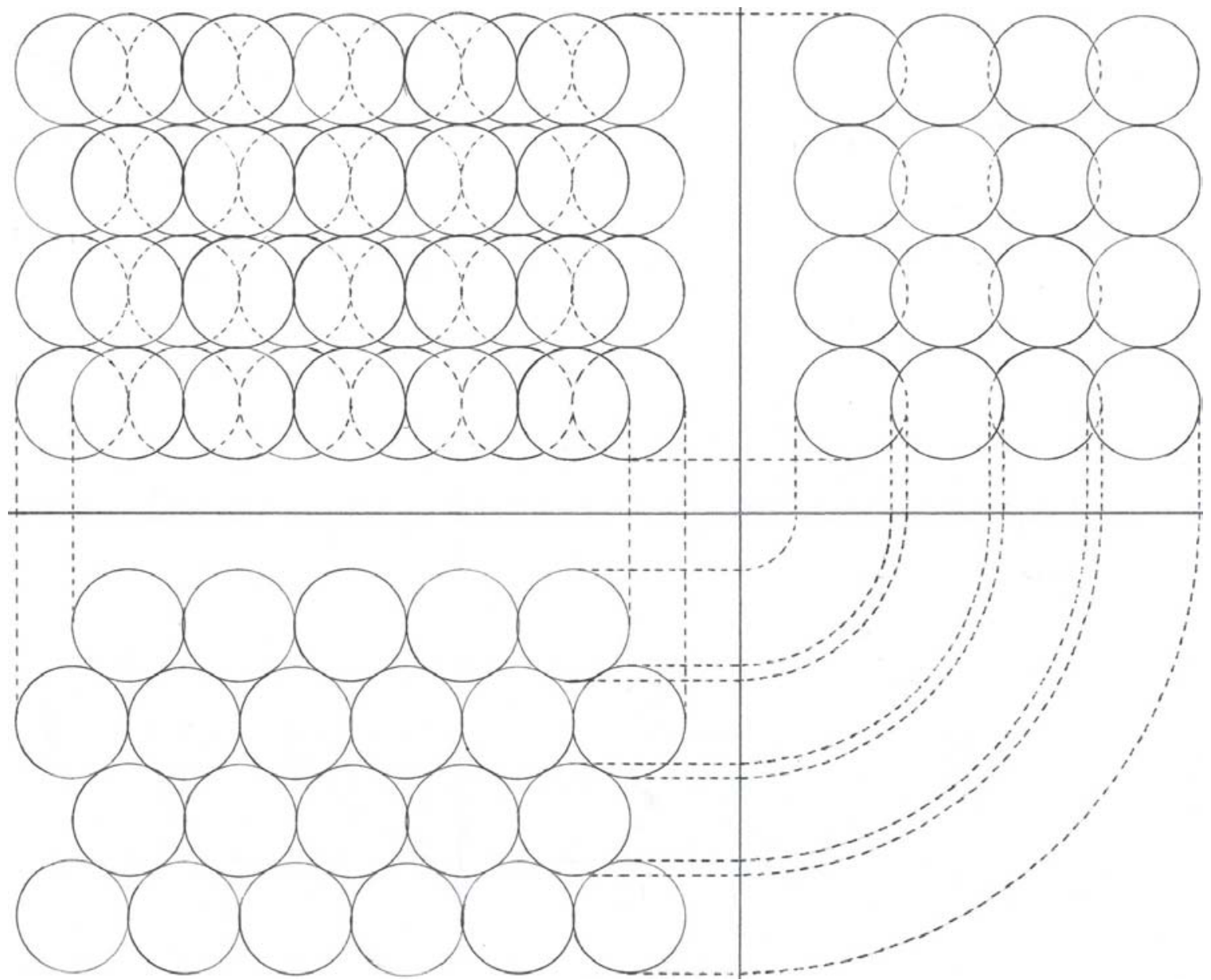

Front view

Figure 3.3. Schematic picture of the packing structure of coordination number 8 (orthorhombic packing structure). 


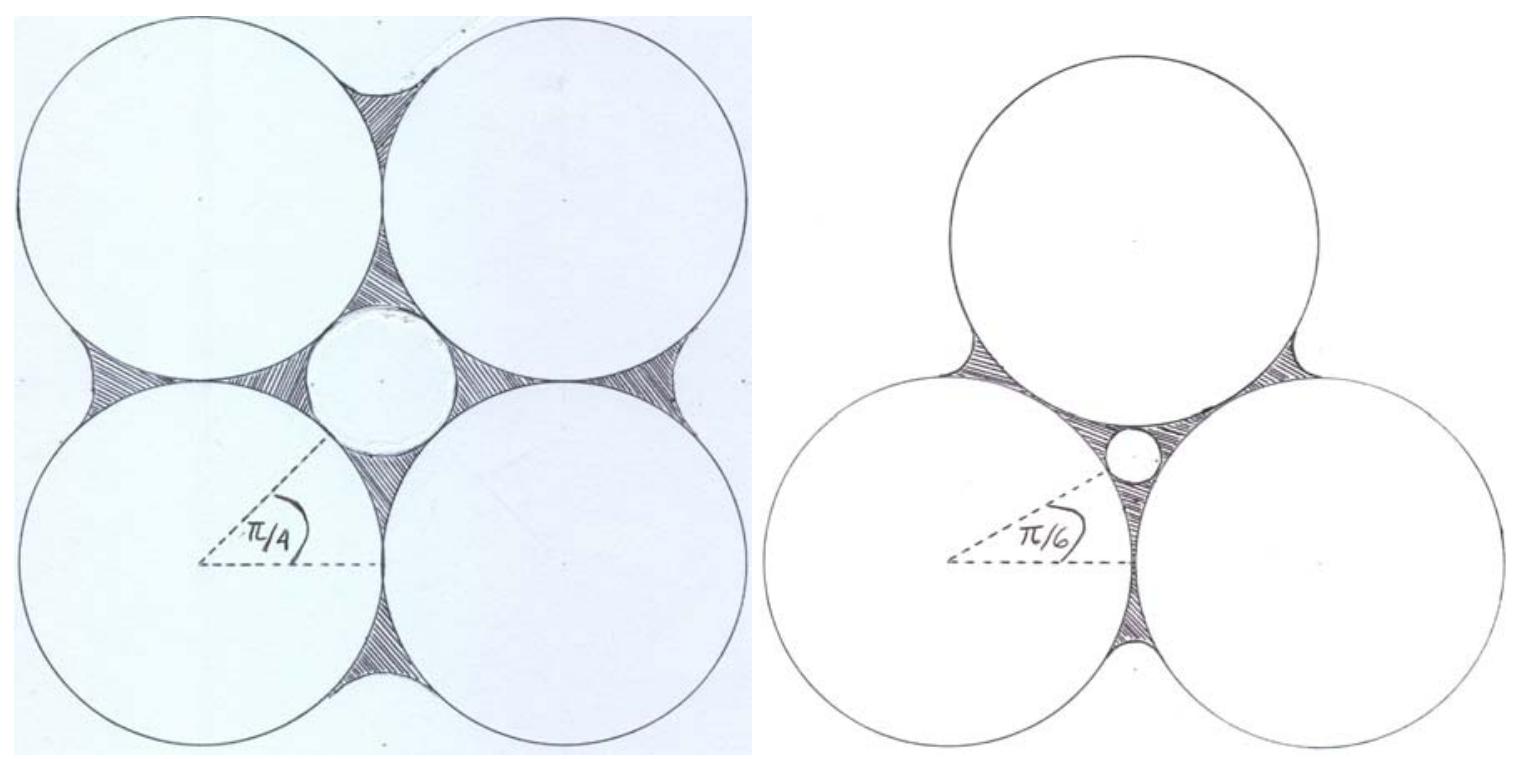

(a)

(b)

Figure 3.4. Schematic picture of unit layers of the orthorhombic packing structure: (a) Unit layer of top/bottom side of view; or left/right side of view if the plane of view is rotated as far $30^{\circ}$; (b) Unit layer of the front/back side of view.

Figure 3.4 tells us the maximum value of $2 \theta$ when the water bridges start to touch each other. We can see here that the water bridges start to touch each other at $2 \theta=\pi / 4$ in some parts and $2 \theta=\pi / 6$ in the other parts of the bed. It means, in average, the maximum value of $2 \theta$ before the water bridges touch each other is between $\pi / 6$ and $\pi / 4$. Using Equations (3.10) and (3.11) and data in Table 3.1, we can calculate the corresponding values of $S$ as shown in Table 3.2. For our experiments, we chose $S=0.30$, which is in the range of $S$ according Table 3.2. The corresponding values of all the parameters in the model of single water bridge for $S=0.30$ are shown in Table 3.3. 
TABLE 3.2

Relative Saturation of Void by Water $S$ at Two Values of $2 \theta$

\begin{tabular}{|c|c|c|c|}
\hline$d_{p}, \mu \mathrm{m}$ & $2 \theta$, radian & $M$ & $S$ \\
\hline 240 & $\pi / 6=0.5236$ & 0.0230 & 0.0891 \\
\cline { 2 - 4 } & $\pi / 4=0.7854$ & 0.0895 & 0.3469 \\
\hline 510 & $\pi / 6=0.5236$ & 0.0229 & 0.0932 \\
\cline { 2 - 4 } & $\pi / 4=0.7854$ & 0.0891 & 0.3626 \\
\hline
\end{tabular}

TABLE 3.3

Parameters in the Model of a Single Water Bridge

\begin{tabular}{|c|c|c|c|c|c|c|c|}
\hline $\begin{array}{c}d_{p}, \\
\mu \mathrm{m}\end{array}$ & $S$ & $M$ & $\begin{array}{c}2 \theta, \\
r_{1} \times 10^{5},\end{array}$ & $\begin{array}{c}r_{2} \times 10^{5}, \\
\mathrm{~m}\end{array}$ & $\begin{array}{c}A_{\text {single }} \times 10^{8}, \\
\mathrm{~m}\end{array}$ & $\begin{array}{c}V_{\text {single }} \times 10^{13} \\
\mathrm{~m}^{3}\end{array}$ & \\
\hline 240 & 0.30 & 0.0774 & 0.7515 & 4.4227 & 6.7891 & 3.3094 & 3.4580 \\
\hline 510 & 0.30 & 0.0737 & 0.7414 & 9.0753 & 14.2743 & 14.4524 & 31.7433 \\
\hline
\end{tabular}

\subsection{Physical model of MGH/PGH formation}

The mass transfer model that is the base of the physical model of MGH/PGH formation (the water-bridge-dispersion-model) is shown in Figure 3.5. At the gas-liquid interface, there was equilibrium between methane/propane in gas phase and dissolved methane/propane in the water (1) phase. The solute methane/propane diffused from methane/propane (g)-water (1) interface to the water (1)-MGH/PGH (s) interface, and formed more $\mathrm{MGH} / \mathrm{PGH}$ at the water (1)- MGH/PGH (s) interface. 


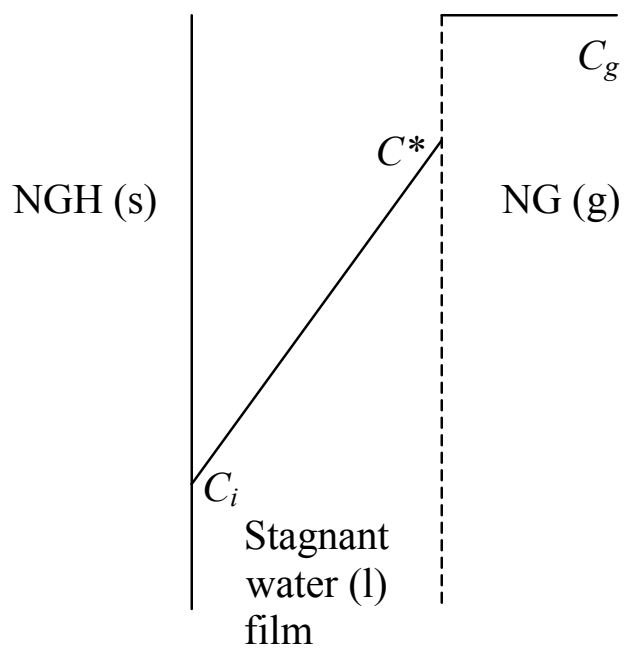

Figure 3.5. Mass transfer model of $\mathrm{MGH} / \mathrm{PGH}$ formation.

The mass transfer model of MGH/PGH formation should give two liquid films, i.e. liquid film at the gas-water (1) interface and liquid film at the water (1)-MGH/PGH (s) interface. According to the calculation results presented in Table 3.3 for $S=0.30$, the normal distances between water bridge meniscus and the point of contact between two glass beads $\left(r_{2}\right)$ are $6.7891 \times 10^{-5}$ and $14.2743 \times 10^{-5} \mathrm{~m}$ for glass bead diameters 240 and $510 \mu \mathrm{m}$ respectively. These values are in the order of liquid film thickness whose typical value for many mass transfer processes is around $10^{-4} \mathrm{~m}$ (Cussler, 1997). Consequently, the whole single water bridge acted as single liquid film. In other words, in our experimental condition, instead of two liquid films, we had only one liquid film that lay between gas-water (1) interface and water (1)-MGH/PGH (s) interface, as shown in Figure

\section{5 .}

Furthermore, the derivation of the physical model of $\mathrm{MGH} / \mathrm{PGH}$ formation is as follows. The derivation is based on the quasi steady state assumption that there is no accumulation of dissolved methane/propane in each step of reaction. Hence, the rate of 
each step of the reaction is equal to the rate of methane/propane consumption from the gas phase.

The equilibrium between methane/propane in gas phase and dissolved methane/propane in the water (1) phase at the gas-water (1) interface can be expressed as Equation (3.12).

$$
C_{g}=H C^{*}
$$

in which

$C_{g}=$ concentration of methane/propane in the gas phase

$$
\begin{aligned}
& C^{*}=\text { concentration of dissolved methane/propane in water (1) phase at the gas- } \\
& \text { water (1) interface } \\
& H=\text { partition factor (Henry's constant) of dissolved methane/propane in water (1) } \\
& \text { at the temperature of reaction }
\end{aligned}
$$

Diffusion of the dissolved methane/propane from gas-water (1) interface to the water (1)$\mathrm{MGH} / \mathrm{PGH}$ (s) interface is following Equation (3.13).

$$
\left(-\frac{d n}{d t}\right)=k_{L} A\left(C^{*}-C_{i}\right)
$$

in which

$(-d n / d t)=$ the rate of methane/propane consumption from the gas phase

$k_{L}=$ mass transfer coefficient of methane/propane in the water (1) film

$A=$ surface area of gas-water (1) interface $\approx$ total surface area of MGH/PGH

crystals (by assumption No. 4 in page 26) 
$C_{i}=$ concentration of dissolved methane/propane in water (1) phase at water (1)-

MGH/PGH (s) interface

The rate of MGH/PGH formation on the $\mathrm{MGH} / \mathrm{PGH}$ crystal surface, i.e. the rate of $\mathrm{MGH} / \mathrm{PGH}$ crystal growth, is proportional to $\mathrm{MGH} / \mathrm{PGH}$ crystal surface area and the difference between the dissolved methane/propane concentration $\left(C_{i}\right)$ and its equilibrium concentration $\left(C^{e q}\right)$. Then, the rate of MGH/PGH crystal growth can be formulated as Equation (3.14).

$$
\left(-\frac{d n}{d t}\right)=k_{r} A\left(C_{i}-C^{e q}\right)
$$

in which

$$
\begin{aligned}
k_{r}= & \text { the intrinsic reaction rate constant of } \mathrm{MGH} / \mathrm{PGH} \text { formation } \\
C^{e q}= & \text { equilibrium concentration of dissolved methane/propane in water (1) phase } \\
& \text { at water (1)-MGH/PGH (s) interface }
\end{aligned}
$$

The equation of the MGH/PGH crystal growth rate is following the equation of crystal growth rate available in some literatures (e.g. Perry and Green, 1985 p. 19.29; Sloan, 1998a p. 113; etc.).

By combining Equations (3.12) to (3.14), we get Equation (3.15), which is the rate of methane/propane consumption from the gas phase.

$$
\left(-\frac{d n}{d t}\right)=K A\left(\frac{C_{g}}{H}-C^{e q}\right)
$$

where 


$$
\frac{1}{K}=\frac{1}{k_{L}}+\frac{1}{k_{r}}
$$

Here, $K$ is the overall reaction rate constant. At equilibrium, $d n / d t=0$ and $C_{g}=C_{g}^{e q}$. Then, from Equation (3.15), we obtain Equation (3.17).

$$
\frac{C_{g}^{e q}}{H}=C^{e q}
$$

Equation (3.17) can be substituted into Equation (3.15) to get Equation (3.18).

$$
\left(-\frac{d n}{d t}\right)=\frac{K A}{H}\left(C_{g}-C_{g}^{e q}\right)
$$

Furthermore,

$$
C_{g}=\frac{n}{V_{g}}
$$

in which

$$
V_{g}=\text { volume of gas phase inside the reactor }
$$

Using Equation (3.19), we can rewrite Equation (3.18) to obtain Equation (3.20) with assumption that $V_{g}$ is constant.

$$
\left(-\frac{d C_{g}}{d t}\right)=\frac{K A}{H V_{g}}\left(C_{g}-C_{g}^{e q}\right)
$$

$H$ and $C_{g}^{e q}$ are functions of temperature only. Because isothermal conditions could be maintained during the experiments, it meant $H$ and $C_{g}^{e q}$ were also constant. If there is no additional resistance in the reacting system of the $\mathrm{MGH} / \mathrm{PGH}$ formation reaction during 
each experiment, then $K$ should be constant too. $A$ is approximately constant during the reaction, especially in the first minutes of the reaction when $\mathrm{MGH} / \mathrm{PGH}$ crystals do not block significantly methane/propane $(\mathrm{g})$ - water (1) contact. $V_{g}$ is practically constant during the reaction. Hence, we can solve Equation (3.20) to get Equation (3.21).

$$
\ln \left(\frac{C_{g}-C_{g}^{e q}}{C_{g, 0}-C_{g}^{e q}}\right)=-\frac{K A}{H V_{g}} t
$$

in which

$$
\begin{aligned}
& t=\text { time of reaction } \\
& C_{g, 0}=\text { initial concentration of methane/propane in gas phase }
\end{aligned}
$$

Data obtained from the experiments were gas pressure $(P)$ and temperature $(T)$ inside the reactor at various time of reaction. Gas pressure is related to gas concentration by Equation (3.22) with $z$ was predicted by using Lee/Kesler compressibility factor correlation. Here, $R$ is the ideal gas constant. The equation of $z$ takes the form developed by Pitzer et al. as shown in Equation (3.23).

$$
\begin{aligned}
& C_{g}=\frac{P}{z R T} \\
& z=Z^{0}+\omega Z^{1}
\end{aligned}
$$

Data of the three parameters in Equation (3.23), i.e. the acentric factor $(\omega), Z^{0}$, and $Z^{1}$ can be found in reference (Smith, et al., 1996). Both $Z^{0}$ and $Z^{1}$ are functions of reduced pressure $\left(P_{r} \equiv P / P_{c}\right)$ and reduced temperature $\left(T_{r} \equiv T / T_{c}\right)$, with $P_{c}$ and $T_{c}$ are the critical pressure and temperature respectively. Data of $Z^{0}$ and $Z^{1}$ are available in form of a table in the reference. Data of $\omega, P_{c}$, and $T_{c}$ for methane and propane are shown in Table 3.4. 
Then, using Equations (3.22) and (3.23), the pressure and temperature data can be converted into gas concentration data.

\section{TABLE 3.4}

Data of acentric factor $(\omega)$, critical pressure $\left(P_{c}\right)$ and temperature $\left.\left(T_{c}\right)^{*}\right)$

\begin{tabular}{|c|c|c|c|}
\hline & $\omega$ & $P_{c}$, bar & $T_{c}, \mathrm{~K}$ \\
\hline Methane & 0.012 & 45.99 & 190.6 \\
\hline Propane & 0.152 & 42.48 & 369.8 \\
\hline
\end{tabular}

*) (Smith, et al., 1996)

To get the value of the gradient of the line:

$$
\ln \left(\frac{C_{g}-C_{g}^{e q}}{C_{g, 0}-C_{g}^{e q}}\right) \text { vs } t, \text { i.e. }\left(-\frac{K A}{H V_{g}}\right)
$$

we needed to calculate $C_{g}^{e q}$. At a certain temperature of reaction, $C_{g}^{e q}$ is constant and can be calculated using equation (3.24).

$$
C_{g}^{e q}=\frac{P^{e q}}{z R T}
$$

$P^{e q}$ is the equilibrium pressure of $\mathrm{MGH} / \mathrm{PGH}$ formation at temperature of reaction $(T)$. $P^{e q}$ can be predicted using Figure 3.6. 


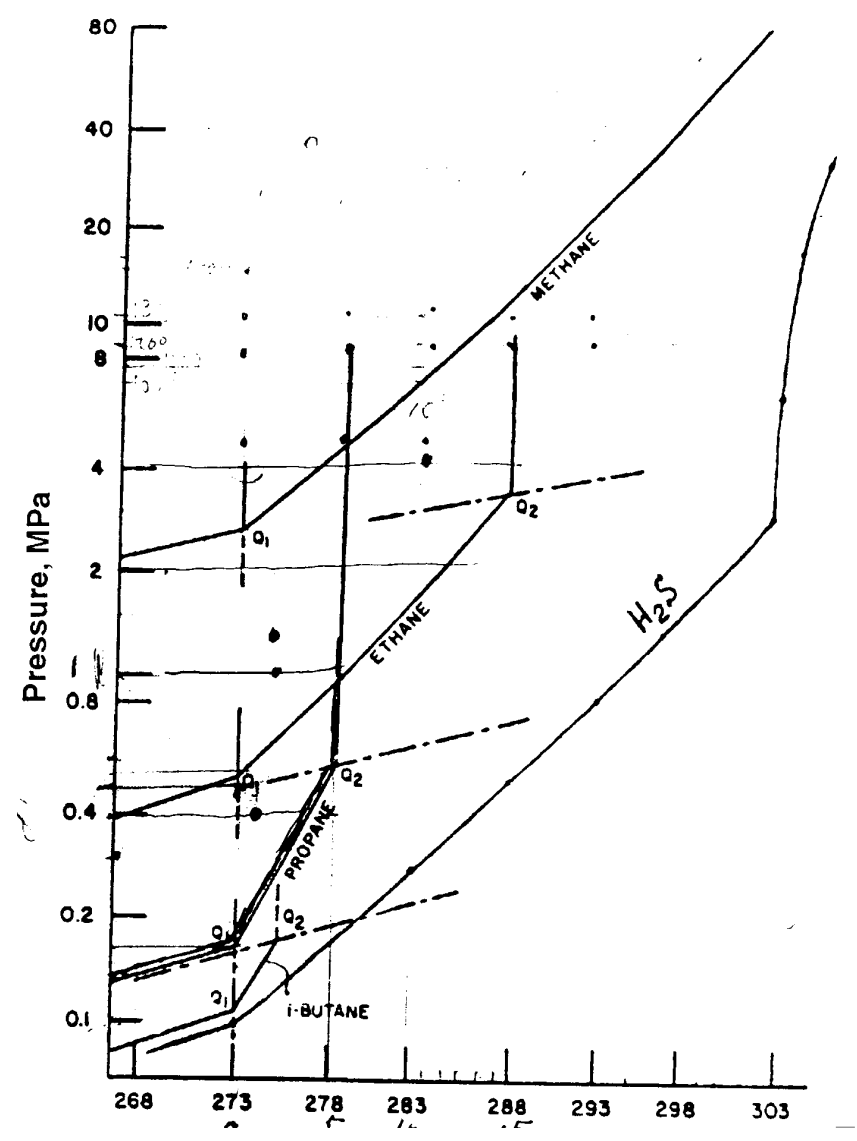

Temperature, $\mathbf{K}$

Figure 3.6. Phase diagrams for some simple NG that form NGH (Katz et al., 1959).

After we converted data of $P$ vs $t$ into data of $\ln \left(\frac{C_{g}-C_{g}^{e q}}{C_{g, 0}-C_{g}^{e q}}\right)$ vs $t$, we can plot the data to get the value of the gradient of the line, i.e. $\left(-\frac{K A}{H V_{g}}\right)$.

Using the model of water bridges, we can calculate $A . V_{g}$ was measured in the experiments. Data of Henry's constants $(H)$ are available in the literatures. At temperature of reaction $(T=273.5 \mathrm{~K}), H^{*}$ of methane is $2.24 \times 10^{4} \mathrm{~atm} / \mathrm{mole}$ fraction of 
dissolved methane in water (1) (Perry and Green, 1985). Data of $H^{*}$ of propane is available in form of an equation as shown by Equation (3.25) (Carroll and Mather, 1997).

$$
\ln H^{*}=552.65815+0.077514 T-\frac{21334.4}{T}-85.84949 \ln T
$$

with $H^{*}$ is in $\mathrm{kPa} /$ mole fraction of dissolved propane in water (1); and $T$ is in $\mathrm{K}$. Using Equation (3.25), we obtain the value of $H^{*}$ of propane at $273.5 \mathrm{~K}$ is $1.36 \times 10^{6} \mathrm{kPa} / \mathrm{mole}$ fraction of dissolved propane in water $=1.35 \times 10^{4} \mathrm{~atm} / \mathrm{mole}$ fraction of dissolved propane in water. To convert $H^{*}$ into $H$, we did the following calculation. Let $x$ be the mole fraction of dissolved methane/propane in water (1); $c_{w 0}$ be the initial concentration of water; and $M_{H 2 O}$ be the molecular weight of water. Following Equation (3.12), we can write the relation between $H^{*}$ and $H$ as follows.

$$
\begin{aligned}
& \frac{P}{z R T}=H x * c_{w 0}=H x * \frac{\rho_{w}}{M_{H 2 O}} \\
& H^{*}=\frac{P}{x^{*}}=H \frac{\rho_{w^{2}} z T}{M_{H 2 O}}
\end{aligned}
$$

Using Equation (3.26), we can obtain the values of $H$ of methane and propane at $273.5 \mathrm{~K}$, which are 18.0 and 10.8 respectively.

All these data were used to calculate $K$ from the data of gradients of the lines $\left(-\frac{K A}{H V_{g}}\right)$. Then the values of $K$ were compared to the value of $k_{L}$ to determine the rate controlling step. To predict the value of $k_{L}$, we assumed that the diffusion coefficient of dissolved methane/propane in water (1) $\left(D_{L}\right)$ was not changed much by the existence of MGH/PGH in the water (1). For most substances, the diffusion coefficients $\left(D_{L}\right)$ are close to $10^{-9} \mathrm{~m}^{2} / \mathrm{s}$. For example, diffusion coefficients of methane and propane at infinite 
dilution in water at $25^{0} \mathrm{C}$ are $1.49 \times 10^{-9} \mathrm{~m}^{2} / \mathrm{s}$ and $0.97 \times 10^{-9} \mathrm{~m}^{2} / \mathrm{s}$ respectively (Cussler, 1997). $D_{L}$ is approximately proportional to $T$. Hence, the relation between two values of $D_{L}$ of two different $T$ is approximately following Equation (3.27).

$$
\frac{D_{L, T_{1}}}{D_{L, T_{2}}}=\frac{T_{1}}{T_{2}}
$$

Using Equation (3.27) and data of diffusion coefficients of methane and propane at infinite dilution in water at $25^{\circ} \mathrm{C}$, we can predict the values of diffusion coefficients of methane and propane at infinite dilution in water at $273.5 \mathrm{~K}$. The prediction gives the values of diffusion coefficients of methane and propane at infinite dilution in water at $273.5 \mathrm{~K}$ are $1.37 \times 10^{-9} \mathrm{~m}^{2} / \mathrm{s}$ and $0.89 \times 10^{-9} \mathrm{~m}^{2} / \mathrm{s}$ respectively. Furthermore, the relation between $k_{L}$ and $D_{L}$ is shown in Equation (3.28).

$$
k_{L}=\frac{D_{L}}{y_{L}}
$$

with $y_{L}$ is the water (1) film thickness. As we discussed earlier, the values of $r_{2}$, the normal distance between water bridge meniscus and the point of contact of two glass beads, are in the order of liquid film thickness. Hence if we consider $r_{2}$ is equal to the liquid film thickness, we could approximate the value of mass transfer coefficient using Equation (3.29).

$$
k_{L}=\frac{D_{L}}{r_{2}}
$$

Calculation results presented in Table 3.3 for $S=0.30$ gave $r_{2}=6.7891 \times 10^{-5} \mathrm{~m}$ for glass bead diameter $240 \mu \mathrm{m}$ and $r_{2}=14.2743 \times 10^{-5} \mathrm{~m}$ for glass bead diameter $510 \mu \mathrm{m}$. Using the prediction of the values of diffusion coefficients of methane and propane at infinite dilution in water at $273.5 \mathrm{~K}$ and Equation (3.29), we obtain $k_{L}$ has a value around $10^{-5}$ 
$\mathrm{m} / \mathrm{s}$, i.e. approximately $k_{L}=1.49 \times 10^{-5} \mathrm{~m} / \mathrm{s}$ for methane and $k_{L}=0.97 \times 10^{-5} \mathrm{~m} / \mathrm{s}$ for propane. These values are the average values obtained from the calculation using the two data of $r_{2}$ of two glass bead sizes. These values were compared to the values of $K$ to determine the rate controlling step.

As the summary, the physical model of $\mathrm{MGH} / \mathrm{PGH}$ formation in the special packed bed reactor is represented by Equation (3.21).

$$
\ln \left(\frac{C_{g}-C_{g}^{e q}}{C_{g, 0}-C_{g}^{e q}}\right)=-\frac{K A}{H V_{g}} t
$$

This equation was derived based on the following assumptions.

1. There was only one water liquid film between gas-water (1) interface and the surface of MGH/PGH crystals.

2. The surface area of gas-water (1) interface was approximately equal to the total surface area of MGH/PGH crystals.

3. The mass transfer resistance in the gas phase was negligible.

4. The mass transfer of dissolved NG in the water liquid film was in quasi steady state.

5. No heat transfer effects were involved.

6. $C_{g}^{e q}, K, A, H$, and $V_{g}$ were constant.

Furthermore, data obtained from the experiments were gas pressure $(P)$ and temperature $(T)$ at various time of reaction $(t)$. The data were converted into the data of 
$\ln \left(\frac{C_{g}-C_{g}^{e q}}{C_{g, 0}-C_{g}^{e q}}\right)$ vs $t$, with $C_{g}^{e q}$ was calculated based on data of $P^{e q}$ at the corresponding temperature of reaction. Data of $P^{e q}$ were from literature. By plotting the converted data, we got the value of the gradient of the line, i.e. $\left(-\frac{K A}{H V_{g}}\right)$. Using the model of water bridges, we calculated $A$. $V_{g}$ was measured in the experiments. Data of Henry's constants $(H)$ were available in the literatures. Using all these data, we calculated $K$ for each experiment. The values of $K$ were compared to the predicted values of $k_{L}$ to determine the rate controlling step. 


\section{CHAPTER 4}

\section{EXPERIMENTAL}

\subsection{Materials and equipment}

A batch reactor equipped with an $\mathrm{MGH} / \mathrm{PGH}$ dissociation setup as shown in Figure 4.1 was used in the experiments. The reactor was operated isothermally; and dissociation setup was run after the reaction finished. High purity methane $(99.90 \%)$ or propane $(99.90 \%)$ and distilled water were used in the experiments. Each experiment was done using selected glass bead sizes $\left(d_{p}=240\right.$ or $\left.510 \mu \mathrm{m}\right)$, temperature $(T=273.5$ $\mathrm{K})$, and relative saturation of void by water $(S=0.30)$. Data obtained were gas pressure and temperature inside the reactor at various time of reaction.

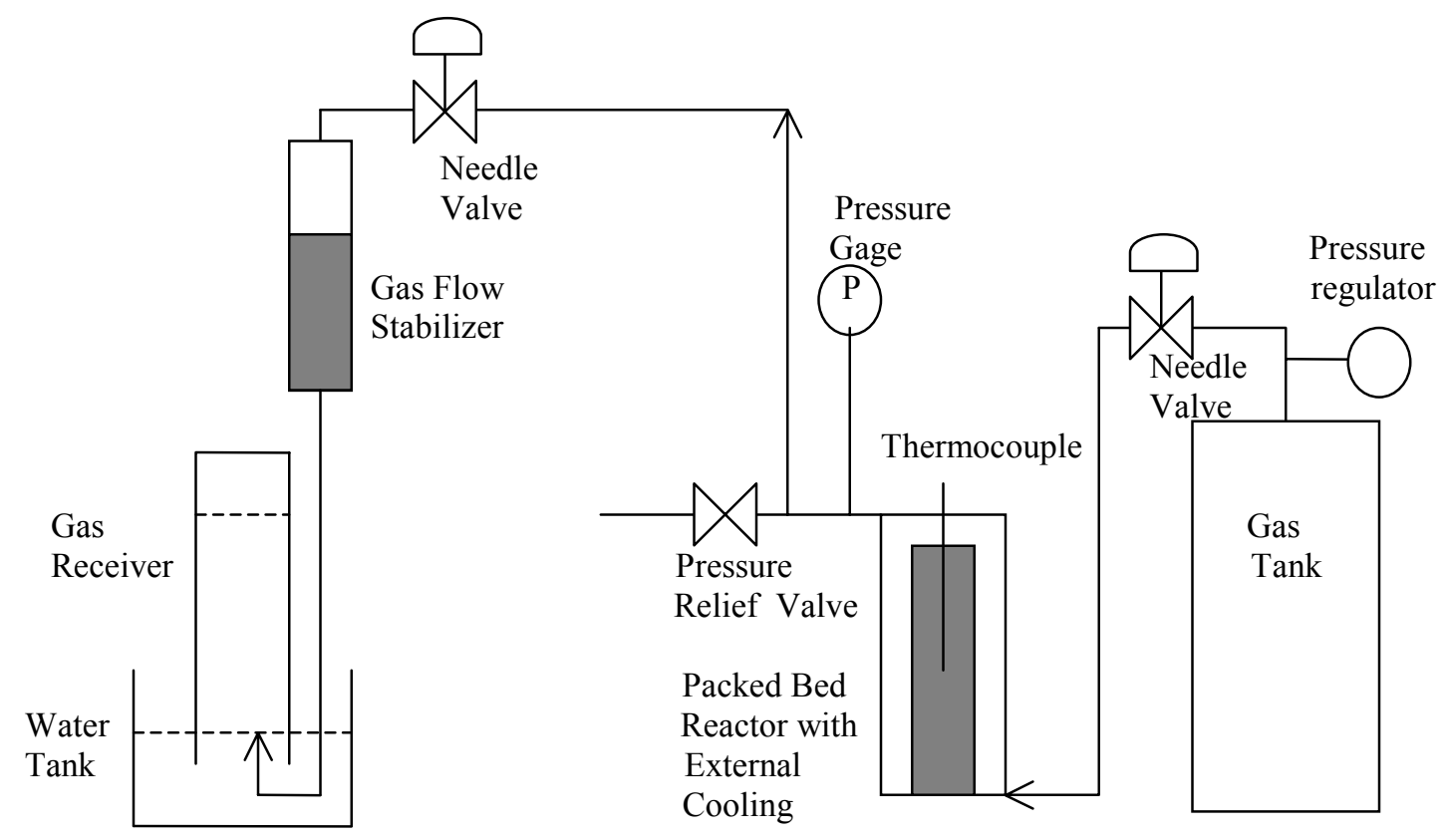

Figure 4.1. Experimental equipment for the synthesis of MGH and PGH equipped with MGH/PGH dissociation setup. 


\subsection{Bed preparation and gas volume $\left(V_{g}\right)$ measurement}

The first step in the experimental procedure was bed preparation. The bed consists of glass beads and water. Known amounts of glass beads and water were mixed thoroughly. The glass beads - water composition was determined by the desired relative saturation of packed bed void by water $(S=0.30)$. The corresponding $M$ for each glass bead size used in the experiments is presented in Table 3.3. Because by definition $M$ is the ratio of water weight to the glass bead weight in the bed, then for a known amount of glass beads, the required water amount could be calculated. The glass beads - water mixture was then fed into a cage made of plastic screen. For MGH experiments, the cage had diameter $2 \mathrm{~cm}$ and length $31.5 \mathrm{~cm}$; while for PGH experiments, the cage had diameter $1.8 \mathrm{~cm}$ and length $30 \mathrm{~cm}$. The cage and its content were placed inside the reactor. MGH reactor was made of a steel pipe of nominal size 1" with outside diameter $1.315 "(=3.34 \mathrm{~cm})$ and wall thickness $0.133 "(=0.338 \mathrm{~cm})$; while PGH reactor was made of a transparent plastic pipe of outside diameter 1 " $(=2.54 \mathrm{~cm})$ and wall thickness 0.1 " (= $0.254 \mathrm{~cm})$. After the bed was ready, the volume of gas phase inside the reactor $\left(V_{g}\right)$ was measured.

To measure $V_{g}$, first the reactor was pressurized to $P_{1}=34.7$ psia with methane/propane. We used methane/propane in order to expel air from the reactor, and replace it with the gas reactant. Then, the gas was released to a graduated cylinder as shown in Figure 4.1 as the gas receiver. The final pressure in the reactor after gas release was $P_{2}=14.7$ psia. The gas pressure inside the graduated cylinder was approximately equal to $P_{2}=14.7$ psia. Here, the remaining water in the graduated cylinder was very little, so its hydrostatic pressure was negligible. Let $\Delta V$ be the volume of the gas released 
to the graduated cylinder. Because $T$ of the gas was constant and $z$ of the gas did not change much, we can derive the equation to calculate $V_{g}$ as follows.

$$
\begin{aligned}
& \left(V_{g}+\Delta V\right) P_{2}=V_{g} P_{1} \\
& V_{g}=\frac{\Delta V P_{2}}{\left(P_{1}-P_{2}\right)}
\end{aligned}
$$

Data of $V_{g}$ of the experiments are presented in Table 4.1.

TABLE 4.1

Experimental Data of Bed Preparation and Gas Volume $\left(V_{g}\right)$ Measurement

\begin{tabular}{|c|c|c|c|c|c|c|}
\hline & Run & $d_{p}, \mu \mathrm{m}$ & $W, \mathrm{~g}$ & $W_{H 2 O}, \mathrm{~g}$ & $A, \mathrm{~m}^{2 *}$ & $V_{g}, \mathrm{~mL}$ \\
\hline \multirow{2}{*}{ MGH } & M102-M114 & 240 & 132.5376 & 10.2802 & 0.9830 & 209.475 \\
\cline { 2 - 7 } & M115-M120 & 510 & 128.9038 & 9.5086 & 0.4327 & 205.8 \\
\hline \multirow{2}{*}{ PGH } & P101-P103 & 240 & 81.4081 & 6.2997 & 0.6028 & 228.585 \\
\cline { 2 - 7 } & P106-P108 & 240 & 67.2982 & 5.1263 & 0.4925 & 263.13 \\
\hline
\end{tabular}

*) $A$ was calculated using Equation (3.7)

From Table 4.1, we can see that although the glass bead amount used in the M115-M120 runs was less than that used in the M102-M114 runs, the $V_{g}$ of the M115M120 runs was smaller than that of the M102-114 runs. It happened because in the M115-M120 runs, we tried to reduce $V_{g}$ by putting dry bigger size glass beads in the gas space inside the reactor. The smaller the $V_{g}$, the larger the pressure drop can be obtained for the same amount of methane converted to MGH. As a result, the accuracy of measurements increased. 


\subsection{MGH/PGH formation experiments}

To start the MGH/PGH formation experiments, the reactor was immersed in icy water contained in a well-insulated plastic container. Using the cooling system, the temperature inside the reactor can be decreased to and maintained at $273.5 \mathrm{~K}$. It means ice formation could be avoided in the reactor. After thermal equilibrium was reached, methane or propane was fed into the reactor. The initial pressure for MGH formation was varied in the range of $42.38-49.28$ bar to investigate the effect of initial pressure on the reaction rate. The initial pressure can not be too high to avoid significant amount of water reacted during gas injection. For PGH formation, the reaction pressure was chosen such that no propane was condensed in the reactor. Data in reference (Perry and Green, 1985) say that at $1.4^{0} \mathrm{C}$, the vapor pressure of propane is $5 \mathrm{~atm}(=5.07 \mathrm{bar})$. Hence, for PGH formation, the initial pressure was varied in the range of $4.18-4.32$ bar.

Continuous monitoring of pressure and temperature of the reactor was done. Using the cooling system, the temperature of the reaction can be maintained isothermally at $273.5 \mathrm{~K}$. After no more pressure drop was observed, MGH/PGH was dissociated by depressurization of the reactor. The dissociation was completed once the reactor pressure reached atmospheric pressure and no more gas bubbled out of the reactor to the gas receiver. After dissociation finished, the reactor was maintained at $273.5 \mathrm{~K}$ and atmospheric pressure to maintain the $\mathrm{MGH} / \mathrm{PGH}$ seeds in the water bridges. The next run of MGH/PGH formation was done in an hour after the dissociation finished. One full cycle of each experiment consisted of one run of $\mathrm{MGH} / \mathrm{PGH}$ formation and one run of MGH/PGH dissociation. By doing the experiments in successive cycles, the induction period could be eliminated from the experiments. 


\section{CHAPTER 5}

\section{RESULTS AND DISCUSSION}

\subsection{Results}

Figures 5.1 to 5.4 show raw experimental data $P$ vs $t$ of MGH formation. We observed that in some runs, there were initial periods in which the pressure decrease was not too sharp. The initial period was well known as the induction period that was followed by the growth period. A new sharp decrease in pressure was the indication of the growth period. The induction period could be eliminated from the experiments after a couple cycles as shown in the figures. MGH formation rate constant $(K)$ was then evaluated for the growth period based on the proposed model.

Using Equations (3.22) to (3.24), we can convert the raw data into NG concentration data in form of $\ln \left(\frac{C_{g}-C_{g}^{e q}}{C_{g, 0}-C_{g}^{e q}}\right)$ vs $t$. The data are presented as Figures 5.5 to 5.23. Linear lines that represent Equation (3.21) were plotted on the figures to include as many points of data during the growth period as possible. The lines should start at the point where growth periods began. The regression equation of each line was presented in each figure. Here, $y \equiv \ln \left(\frac{C_{g}-C_{g}^{e q}}{C_{g, 0}-C_{g}^{e q}}\right), x \equiv t$, and $R^{2}$ is coefficient of correlation. The gradients of the lines are $\left(-\frac{K A}{H V_{g}}\right)$. Using the data of $H$ given in Chapter $3(H$ of 
methane and propane at $273.5 \mathrm{~K}$ are 18.0 and 10.8 respectively), $A$, and $V_{g}$ as given in Table 4.1, we can calculate $K$. The results are given in Table 5.1. 


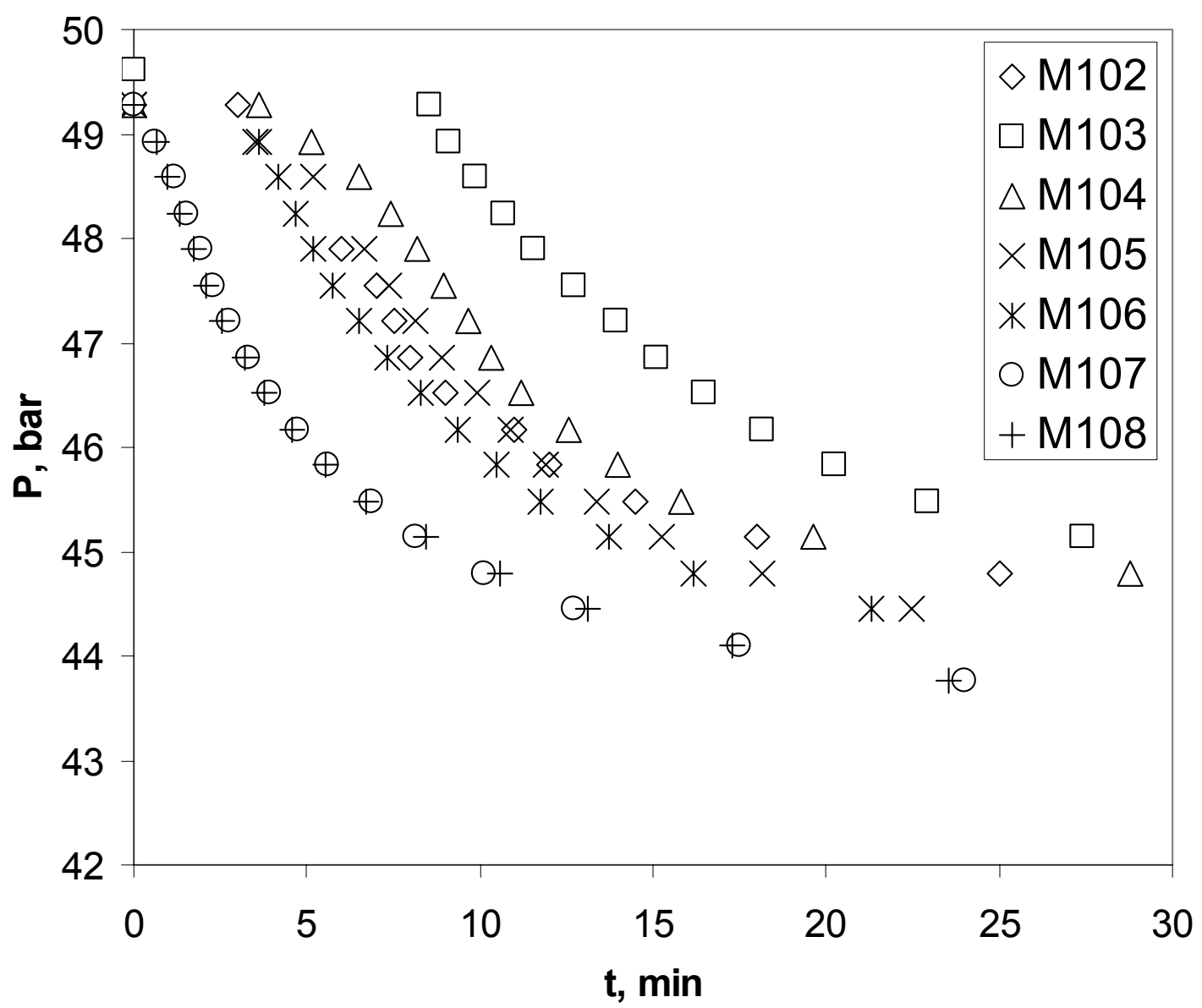

Figure 5.1. Raw data of MGH formation experiments for initial pressure 49.28 bar, $T=273.5 \mathrm{~K}, d_{p}=240 \mu \mathrm{m}$, and $S=0.30$. 


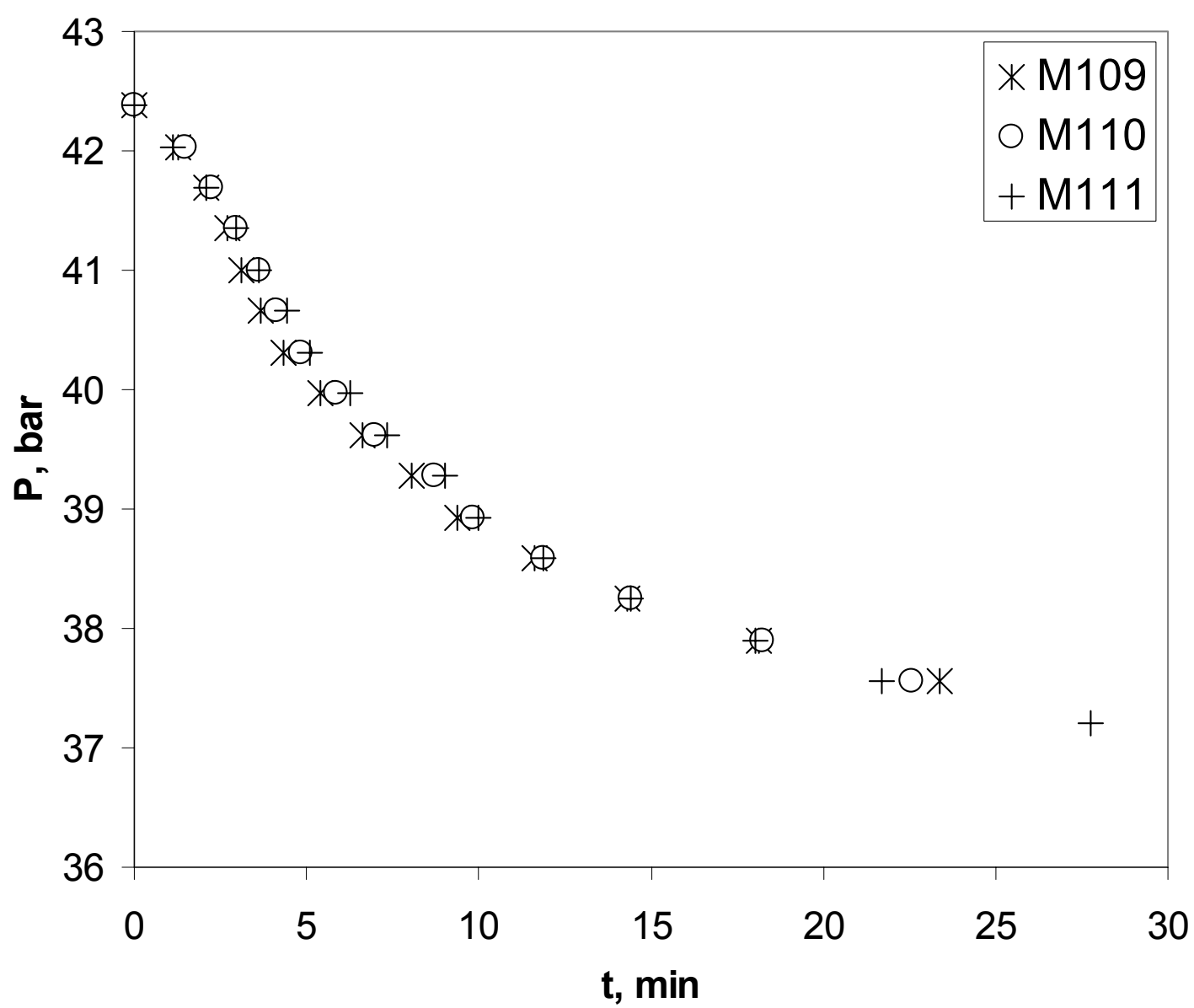

Figure 5.2. Raw data of MGH formation experiments for initial pressure 42.38 bar, $T=273.5 \mathrm{~K}, d_{p}=240 \mu \mathrm{m}$, and $S=0.30$. 


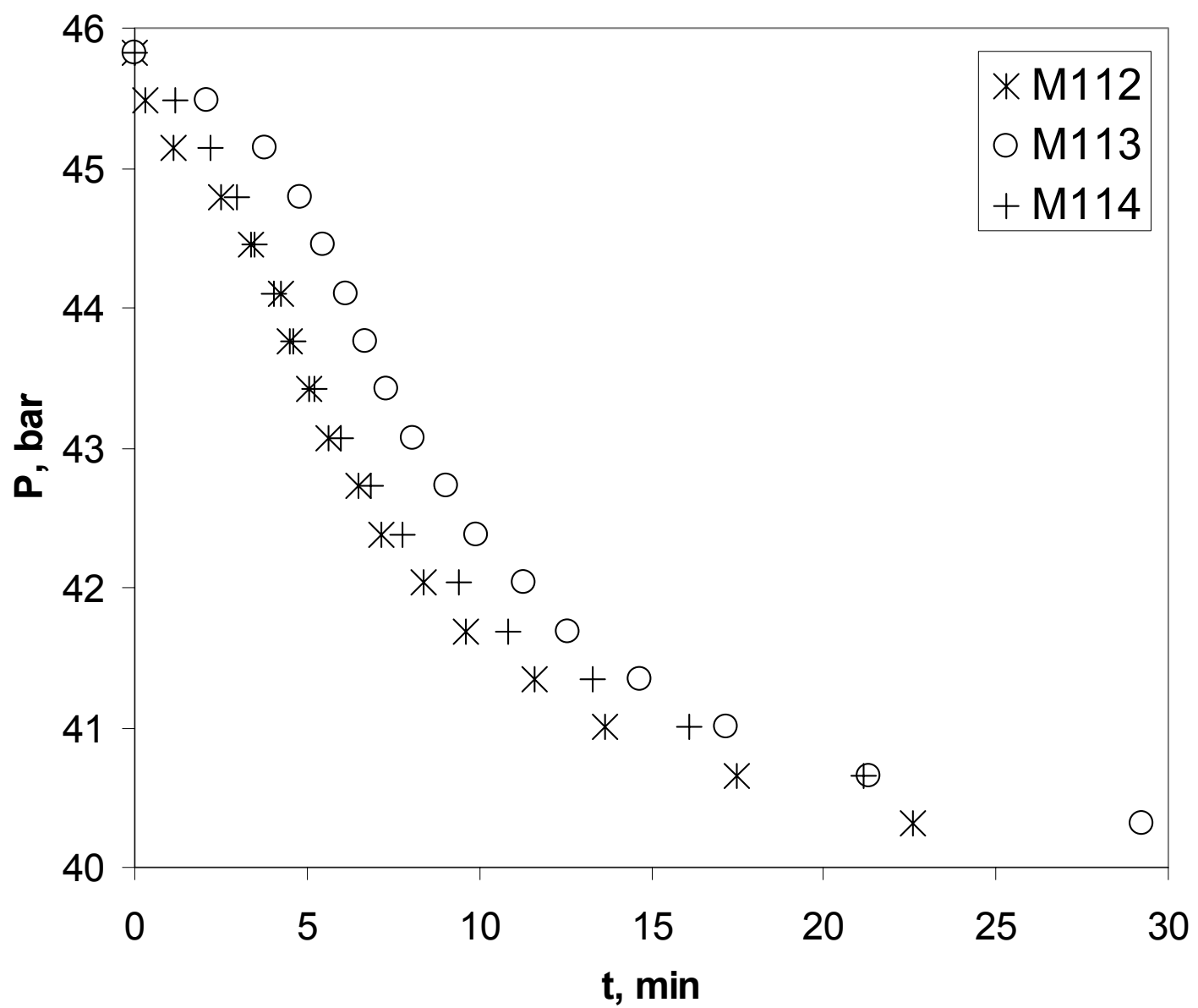

Figure 5.3. Raw data of MGH formation experiments for initial pressure 45.83 bar, $T=273.5 \mathrm{~K}, d_{p}=240 \mu \mathrm{m}$, and $S=0.30$. 


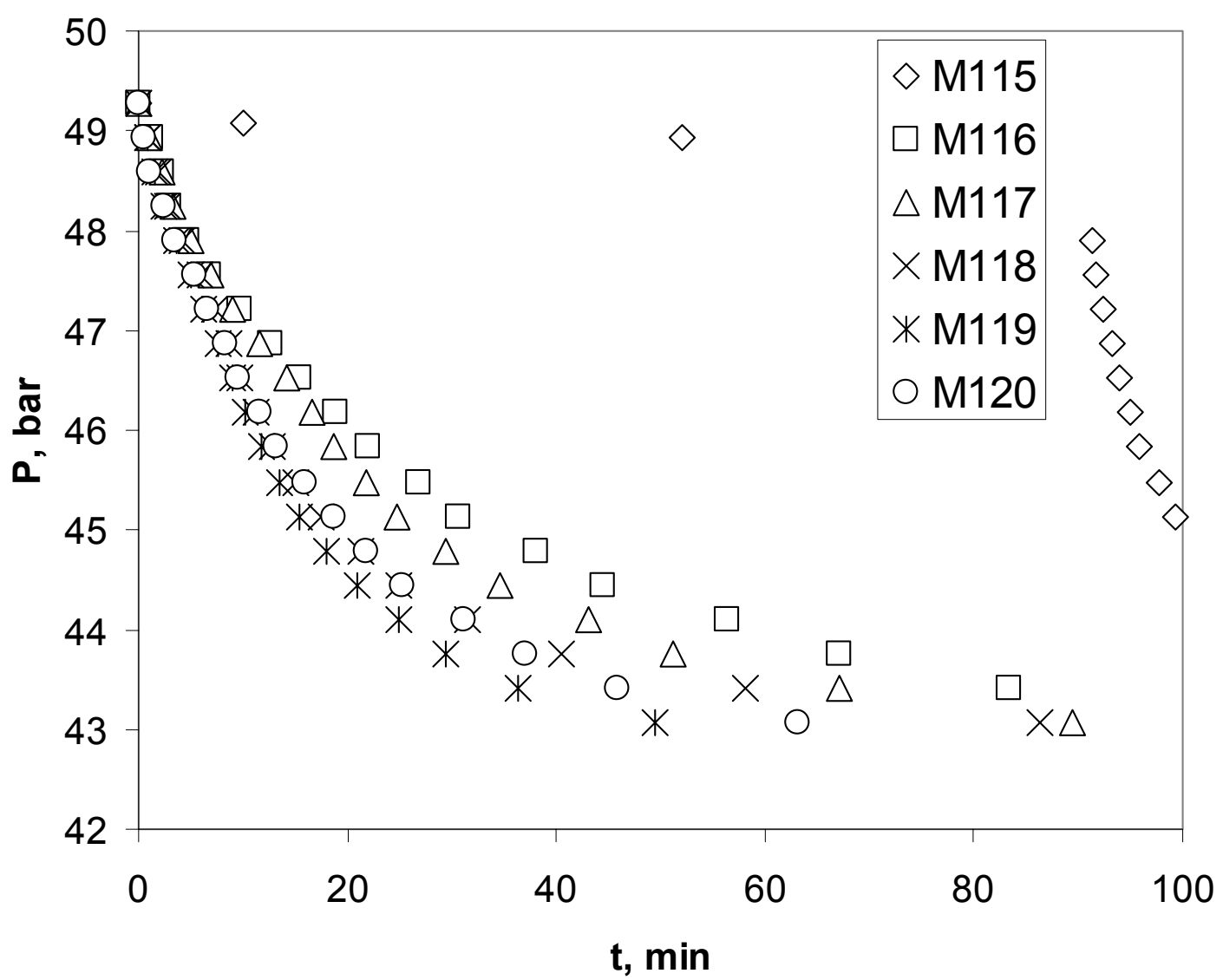

Figure 5.4. Raw data of MGH formation experiments for initial pressure 49.28 bar, $T=273.5 \mathrm{~K}, d_{p}=510 \mu \mathrm{m}$, and $S=0.30$. 


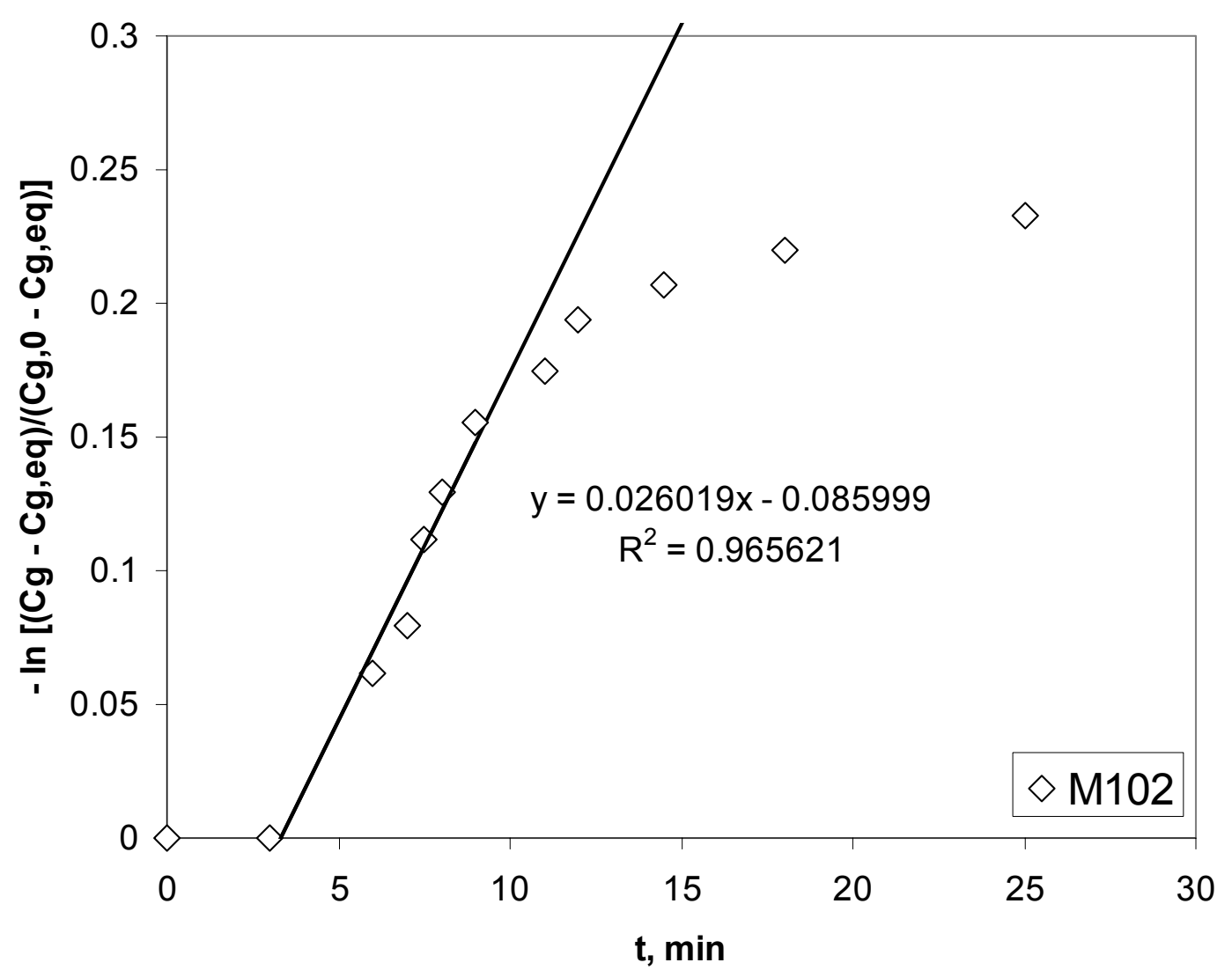

Figure 5.5. Evaluation of the MGH formation experiment Run M102 with initial pressure 49.28 bar, $T=273.5 \mathrm{~K}, d_{p}=240 \mu \mathrm{m}$, and $S$ $=0.30$. 


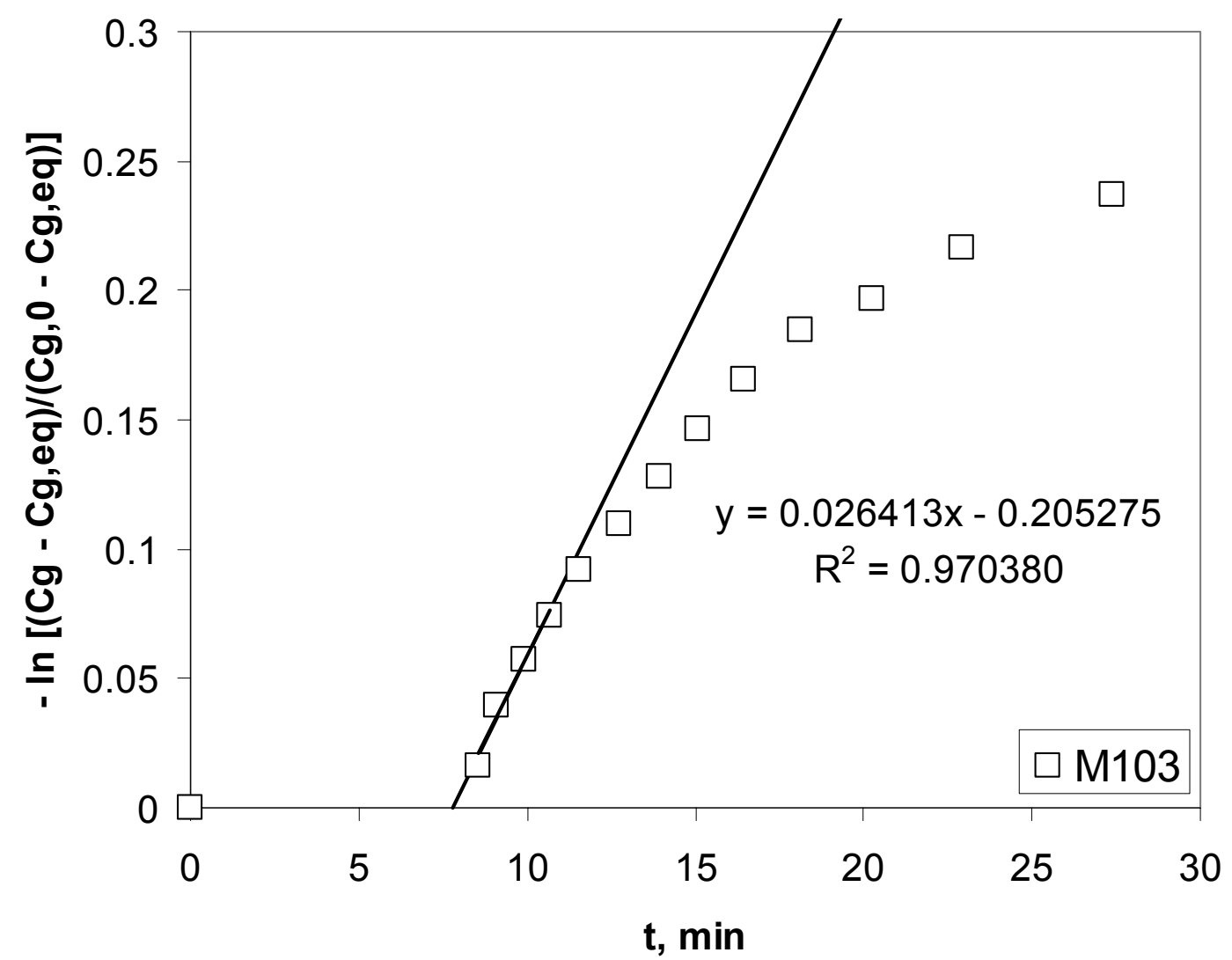

Figure 5.6. Evaluation of the MGH formation experiment Run M103 with initial pressure 49.28 bar, $T=273.5 \mathrm{~K}, d_{p}=240 \mu \mathrm{m}$, and $S$ $=0.30$. 


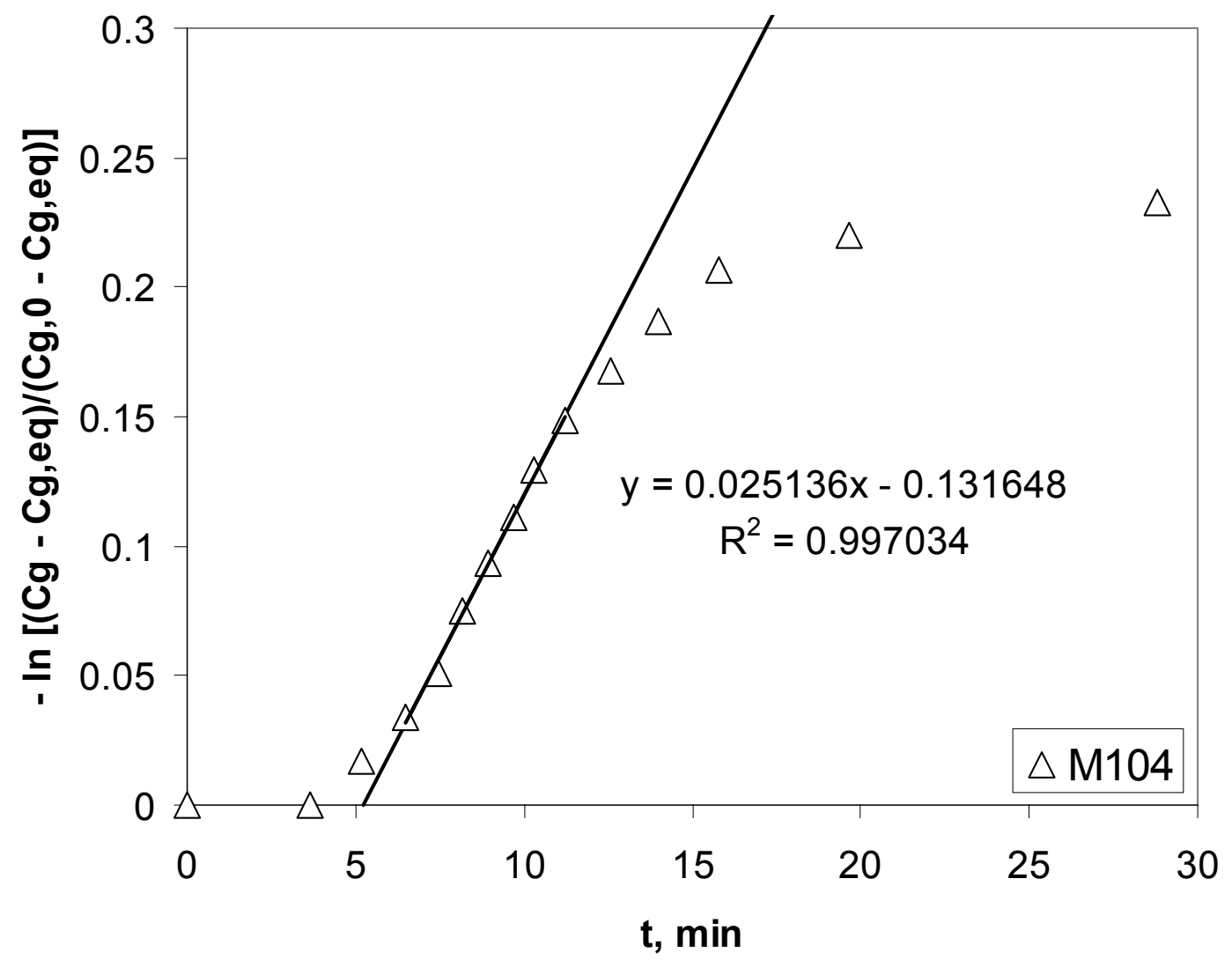

Figure 5.7. Evaluation of the MGH formation experiment Run M104 with initial pressure 49.28 bar, $T=273.5 \mathrm{~K}, d_{p}=240 \mu \mathrm{m}$, and $S$ $=0.30$. 


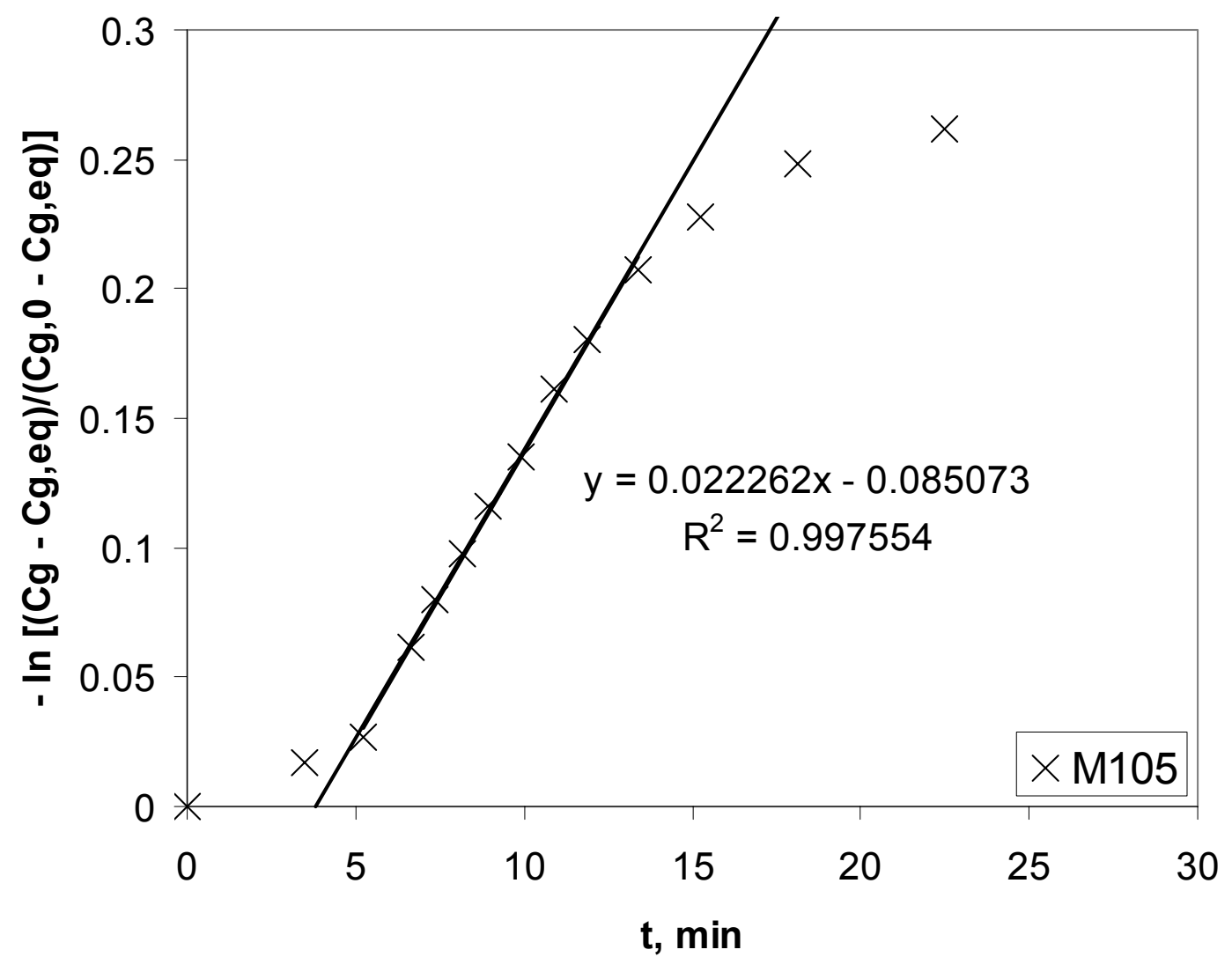

Figure 5.8. Evaluation of the MGH formation experiment Run M105 with initial pressure 49.28 bar, $T=273.5 \mathrm{~K}, d_{p}=240 \mu \mathrm{m}$, and $S$ $=0.30$. 


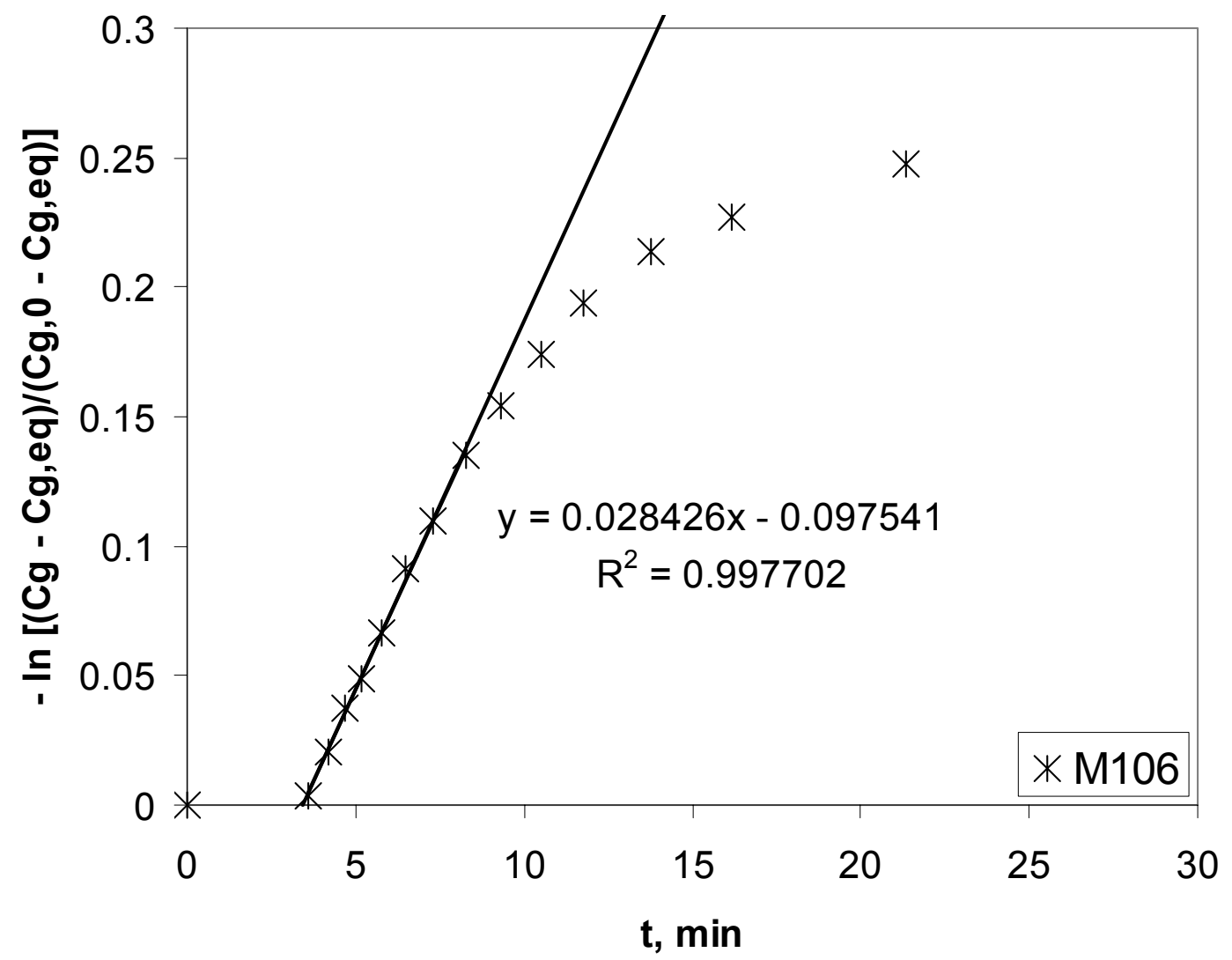

Figure 5.9. Evaluation of the MGH formation experiment Run M106 with initial pressure 49.28 bar, $T=273.5 \mathrm{~K}, d_{p}=240 \mu \mathrm{m}$, and $S$ $=0.30$. 


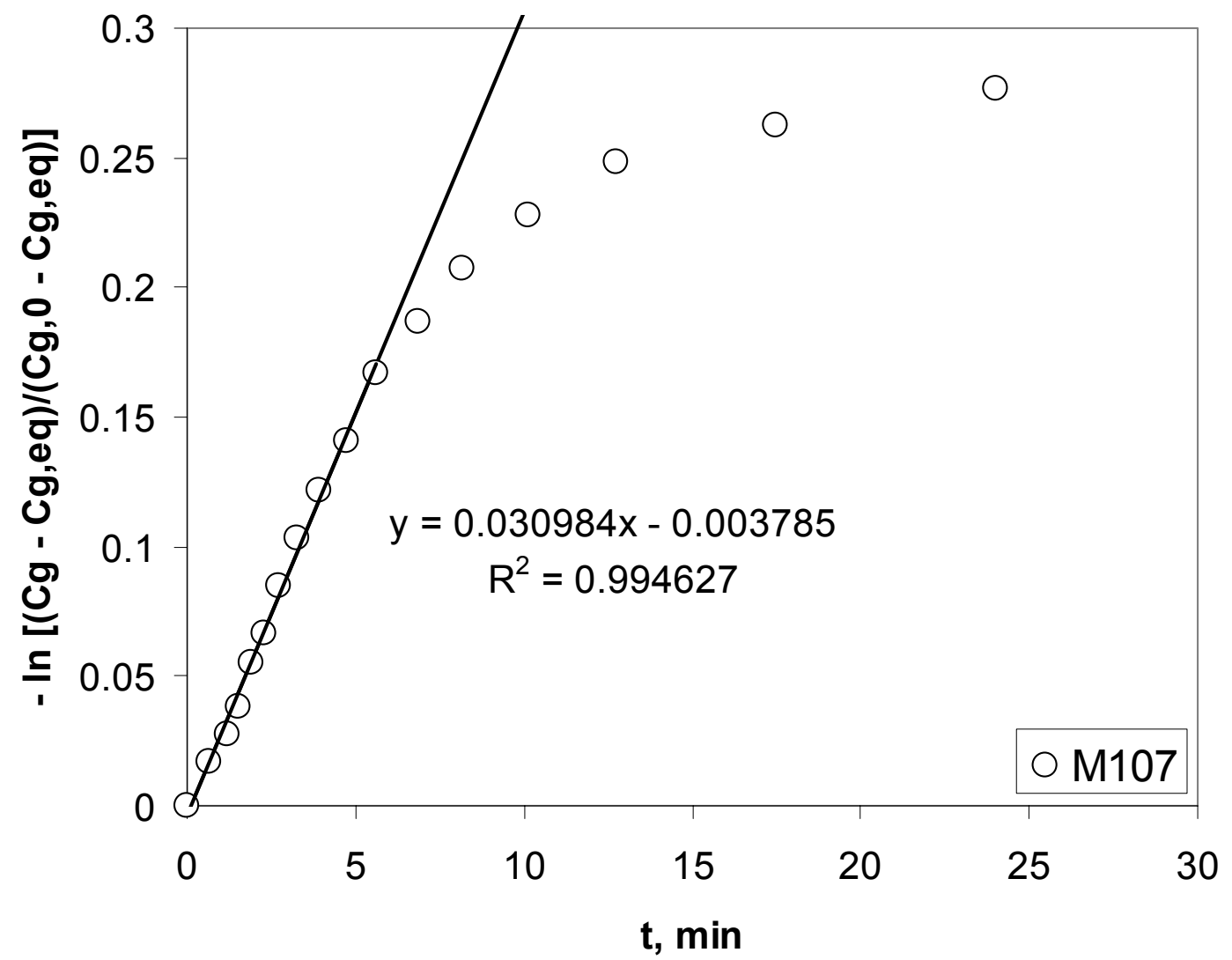

Figure 5.10. Evaluation of the MGH formation experiment Run M107 with initial pressure 49.28 bar, $T=273.5 \mathrm{~K}, d_{p}=240 \mu \mathrm{m}$, and $S$ $=0.30$. 


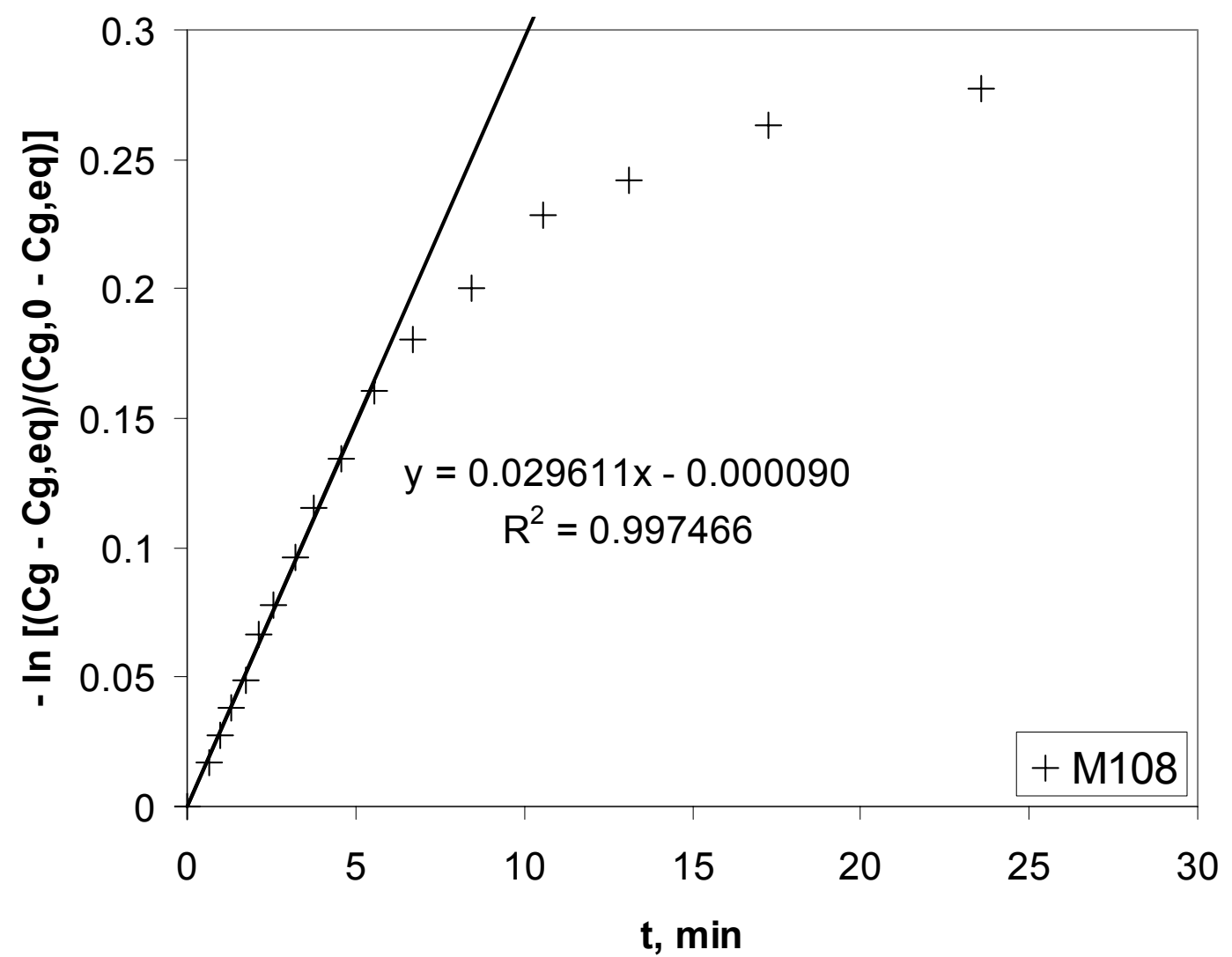

Figure 5.11. Evaluation of the MGH formation experiment Run M108 with initial pressure 49.28 bar, $T=273.5 \mathrm{~K}, d_{p}=240 \mu \mathrm{m}$, and $S$ $=0.30$. 


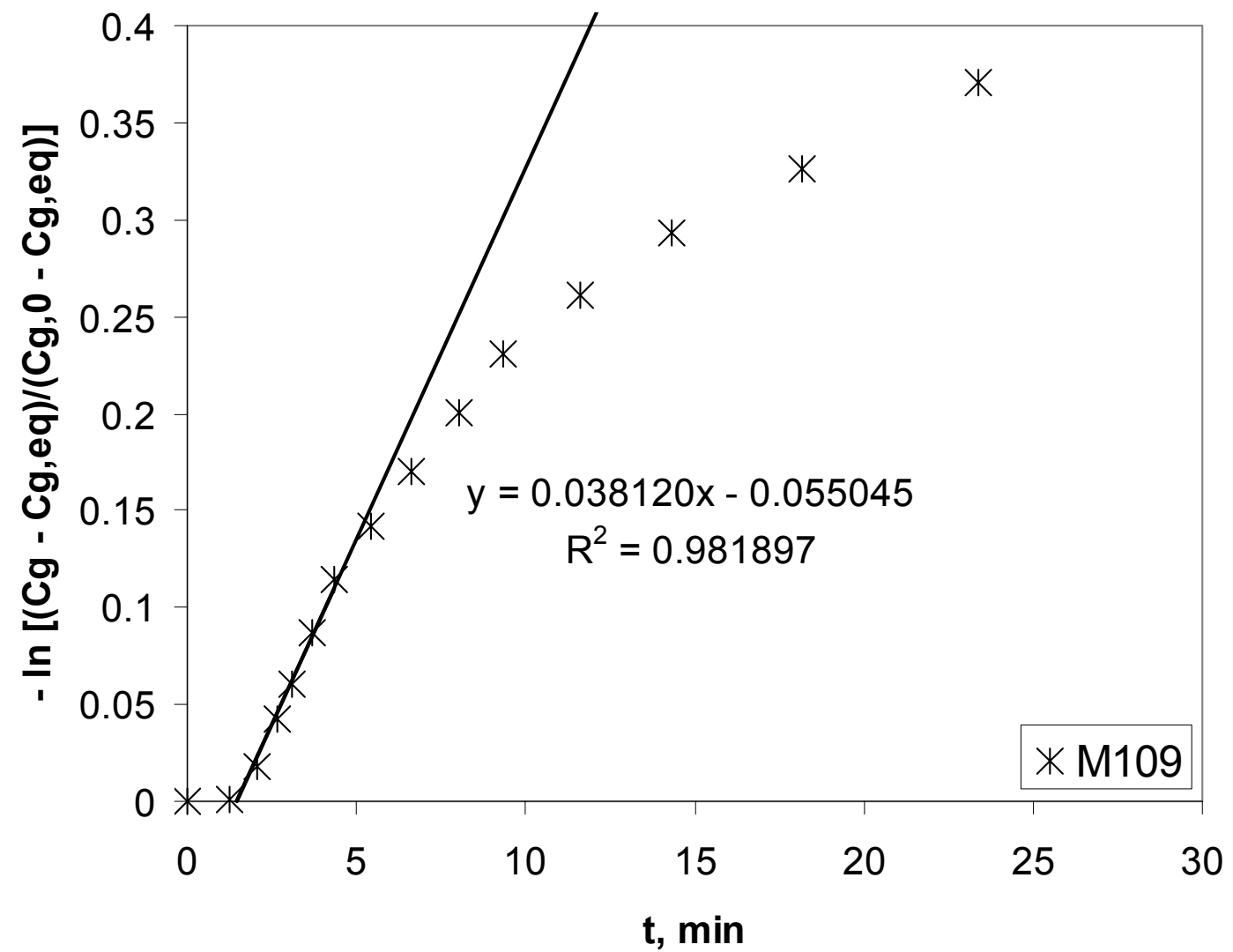

Figure 5.12. Evaluation of the MGH formation experiment Run M109 with initial pressure 42.38 bar, $T=273.5 \mathrm{~K}, d_{p}=240 \mu \mathrm{m}$, and $S$ $=0.30$. 


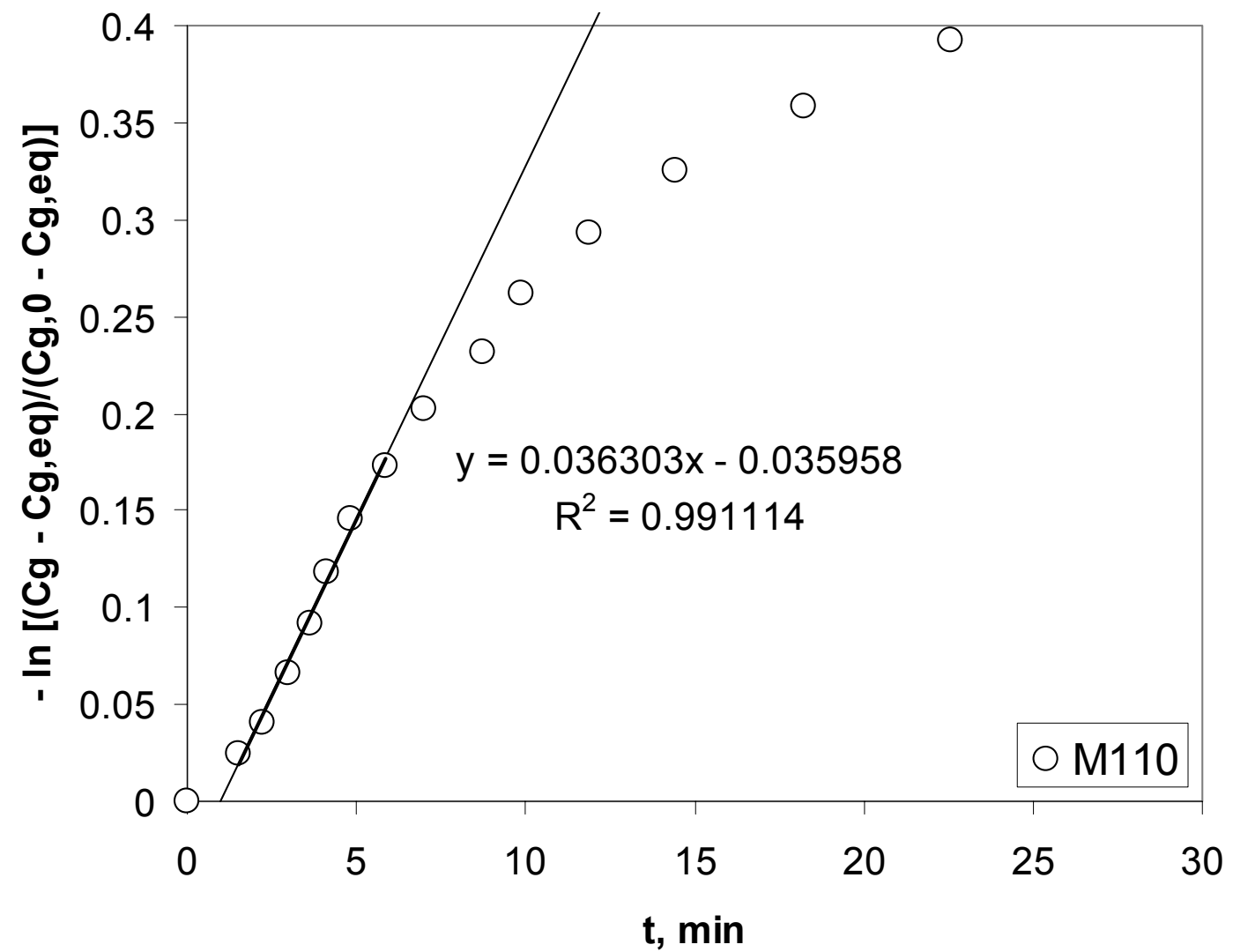

Figure 5.13. Evaluation of the MGH formation experiment Run M110 with initial pressure 42.38 bar, $T=273.5 \mathrm{~K}, d_{p}=240 \mu \mathrm{m}$, and $S$ $=0.30$. 


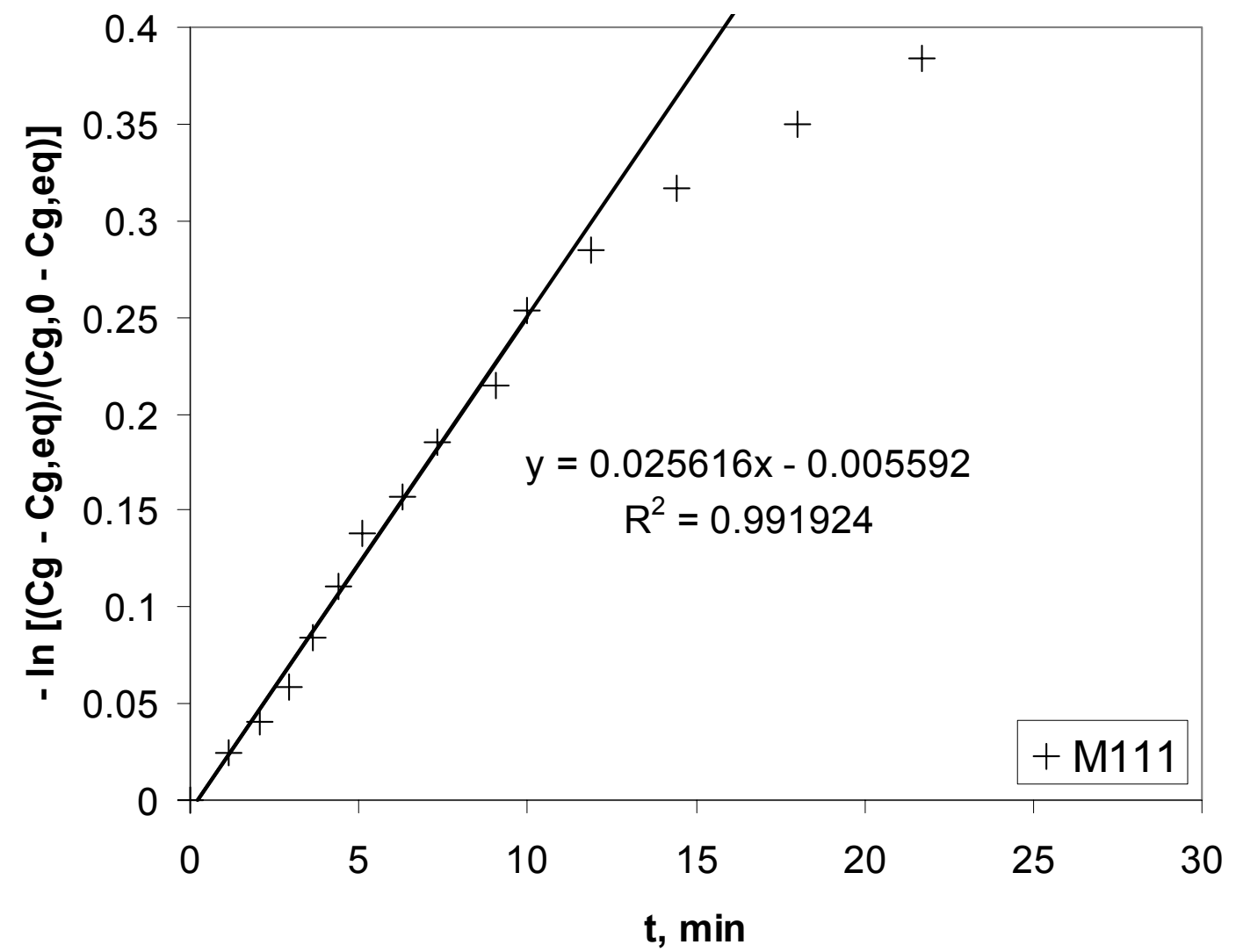

Figure 5.14. Evaluation of the MGH formation experiment Run M111 with initial pressure 42.38 bar, $T=273.5 \mathrm{~K}, d_{p}=240 \mu \mathrm{m}$, and $S$ $=0.30$. 


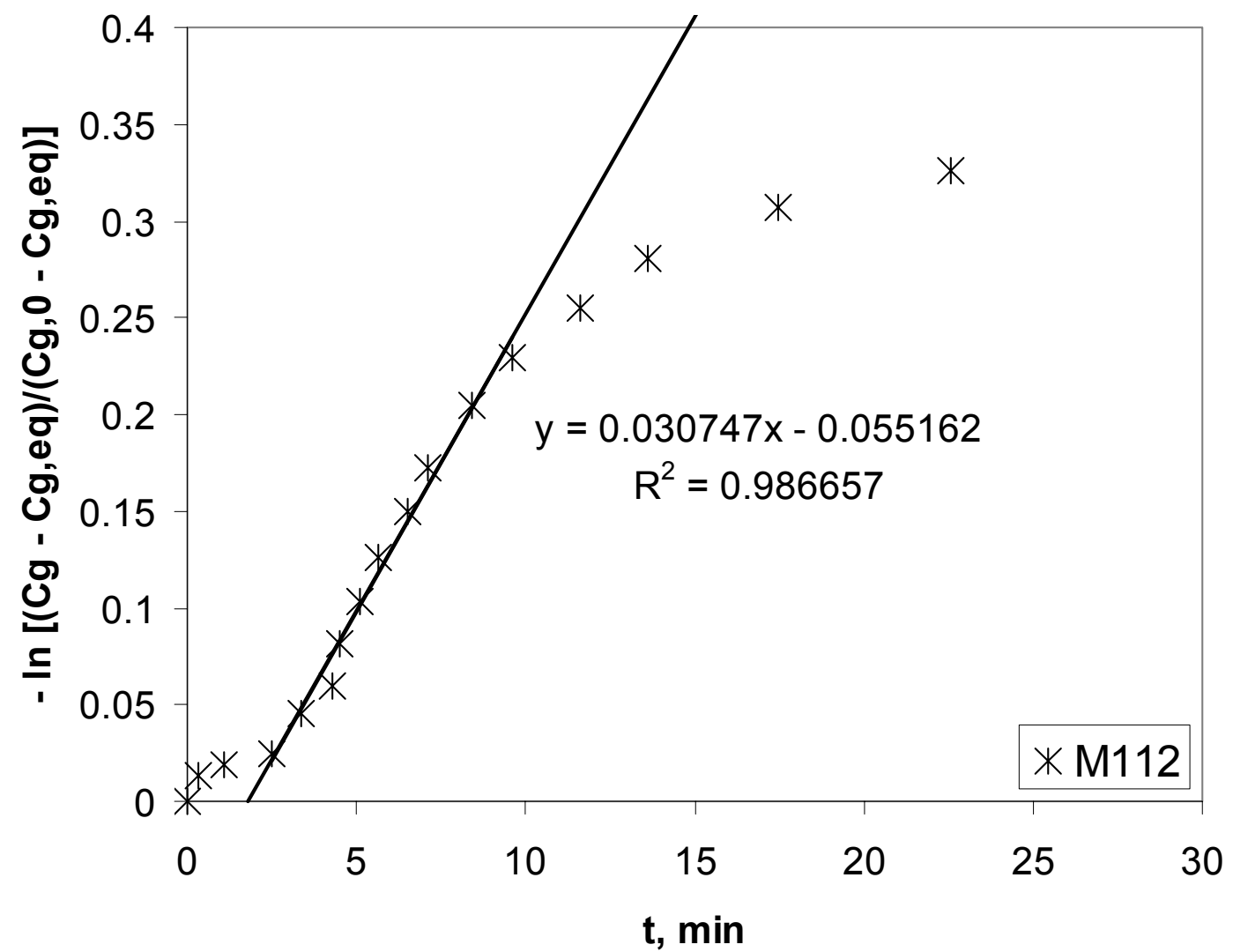

Figure 5.15. Evaluation of the MGH formation experiment Run M112 with initial pressure 45.83 bar, $T=273.5 \mathrm{~K}, d_{p}=240 \mu \mathrm{m}$, and $S$ $=0.30$. 


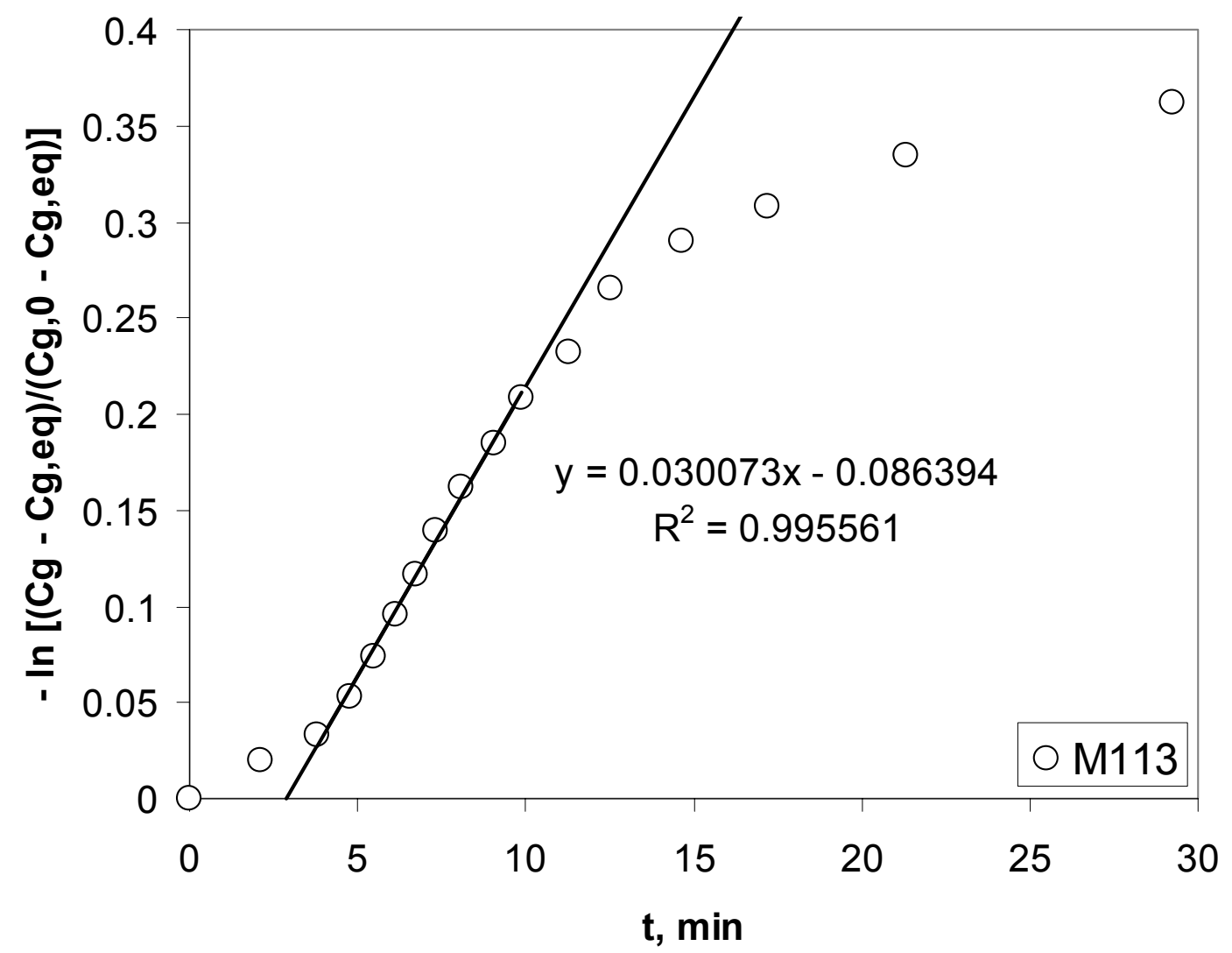

Figure 5.16. Evaluation of the MGH formation experiment Run M113 with initial pressure 45.83 bar, $T=273.5 \mathrm{~K}, d_{p}=240 \mu \mathrm{m}$, and $S$ $=0.30$. 


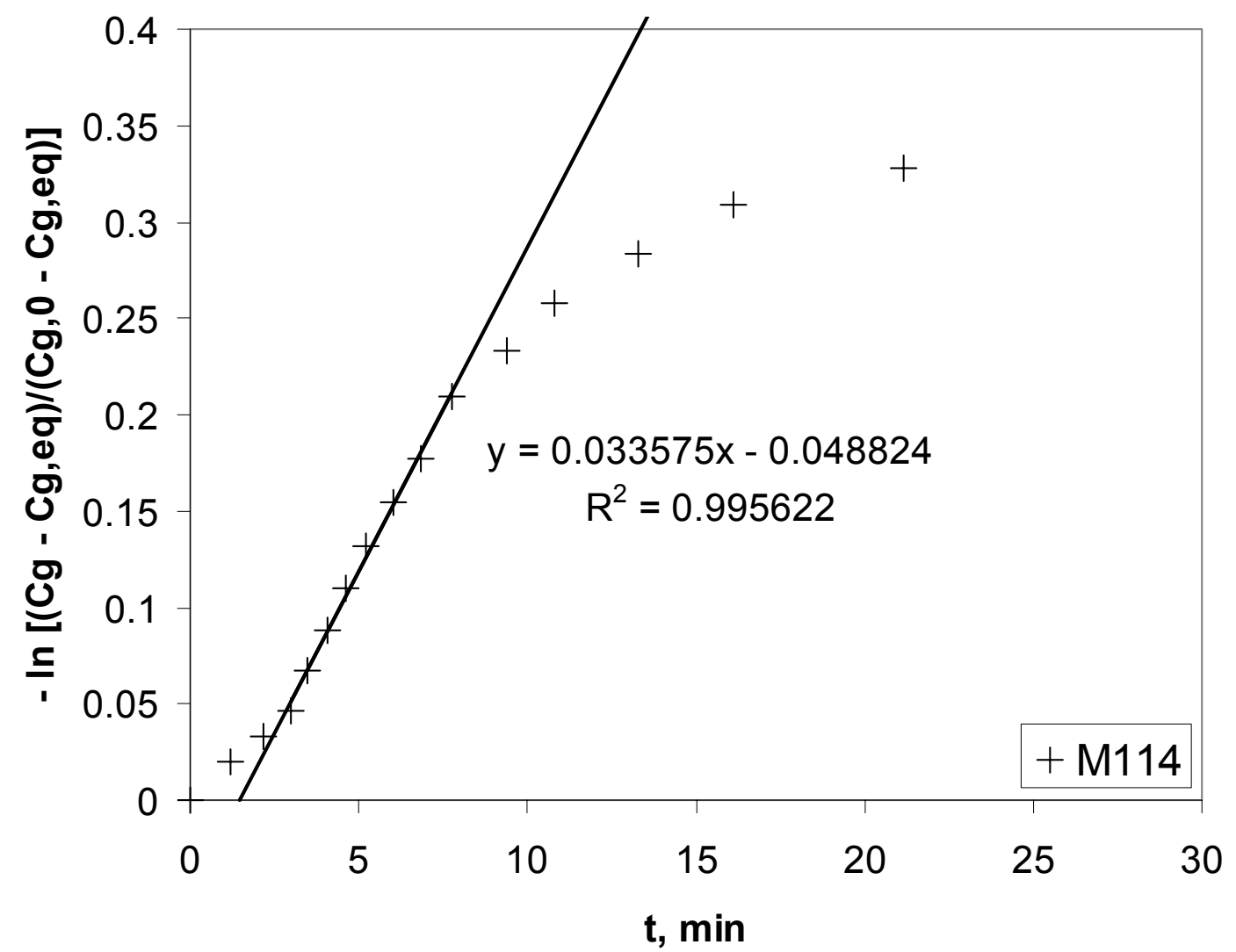

Figure 5.17. Evaluation of the MGH formation experiment Run M114 with initial pressure 45.83 bar, $T=273.5 \mathrm{~K}, d_{p}=240 \mu \mathrm{m}$, and $S$ $=0.30$. 


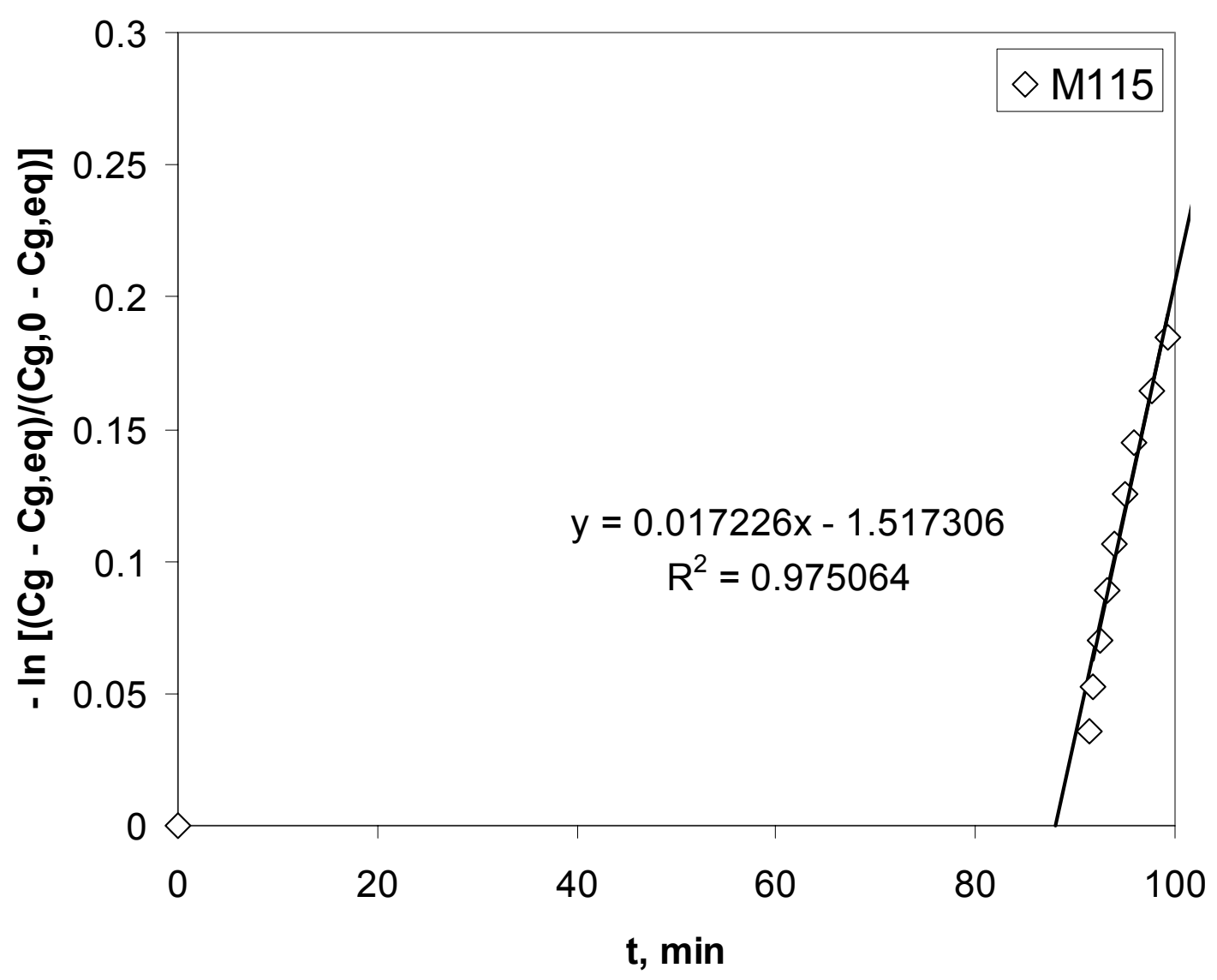

Figure 5.18. Evaluation of the MGH formation experiment Run M115 with initial pressure 49.28 bar, $T=273.5 \mathrm{~K}, d_{p}=510 \mu \mathrm{m}$, and $S$ $=0.30$. 


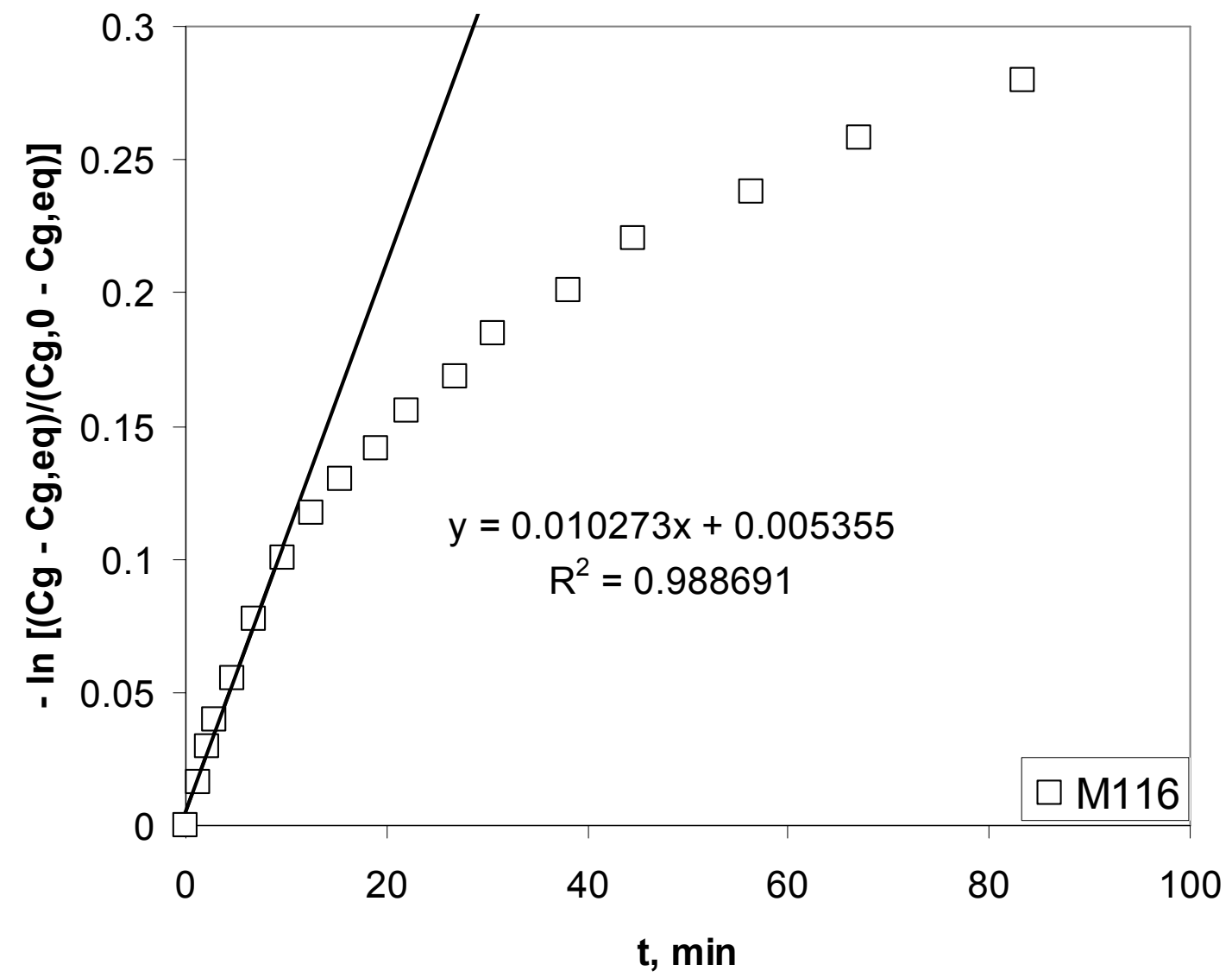

Figure 5.19. Evaluation of the MGH formation experiment Run M116 with initial pressure 49.28 bar, $T=273.5 \mathrm{~K}, d_{p}=510 \mu \mathrm{m}$, and $S$ $=0.30$. 


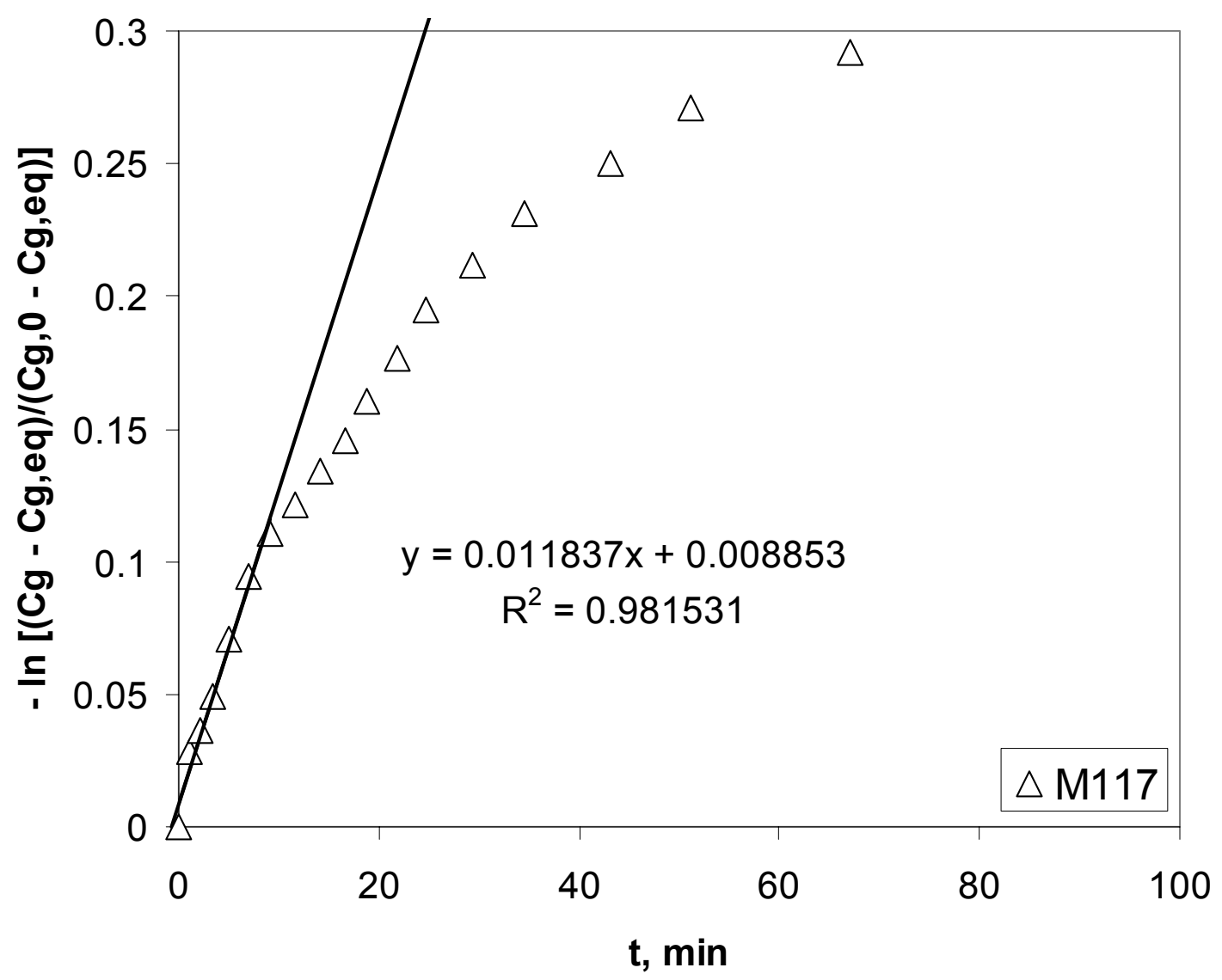

Figure 5.20. Evaluation of the MGH formation experiment Run M117 with initial pressure 49.28 bar, $T=273.5 \mathrm{~K}, d_{p}=510 \mu \mathrm{m}$, and $S$ $=0.30$. 


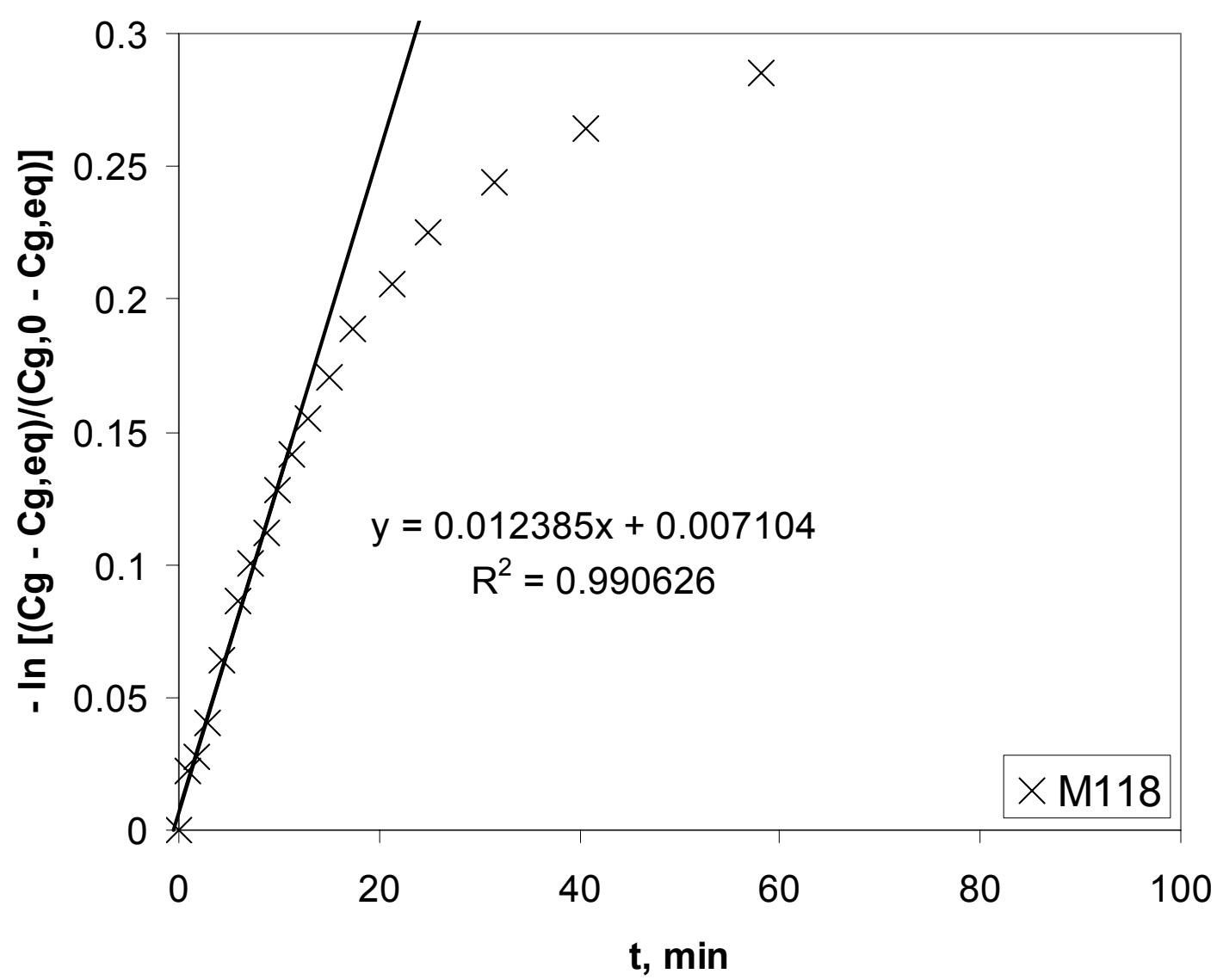

Figure 5.21. Evaluation of the MGH formation experiment Run M118 with initial pressure 49.28 bar, $T=273.5 \mathrm{~K}, d_{p}=510 \mu \mathrm{m}$, and $S$ $=0.30$. 


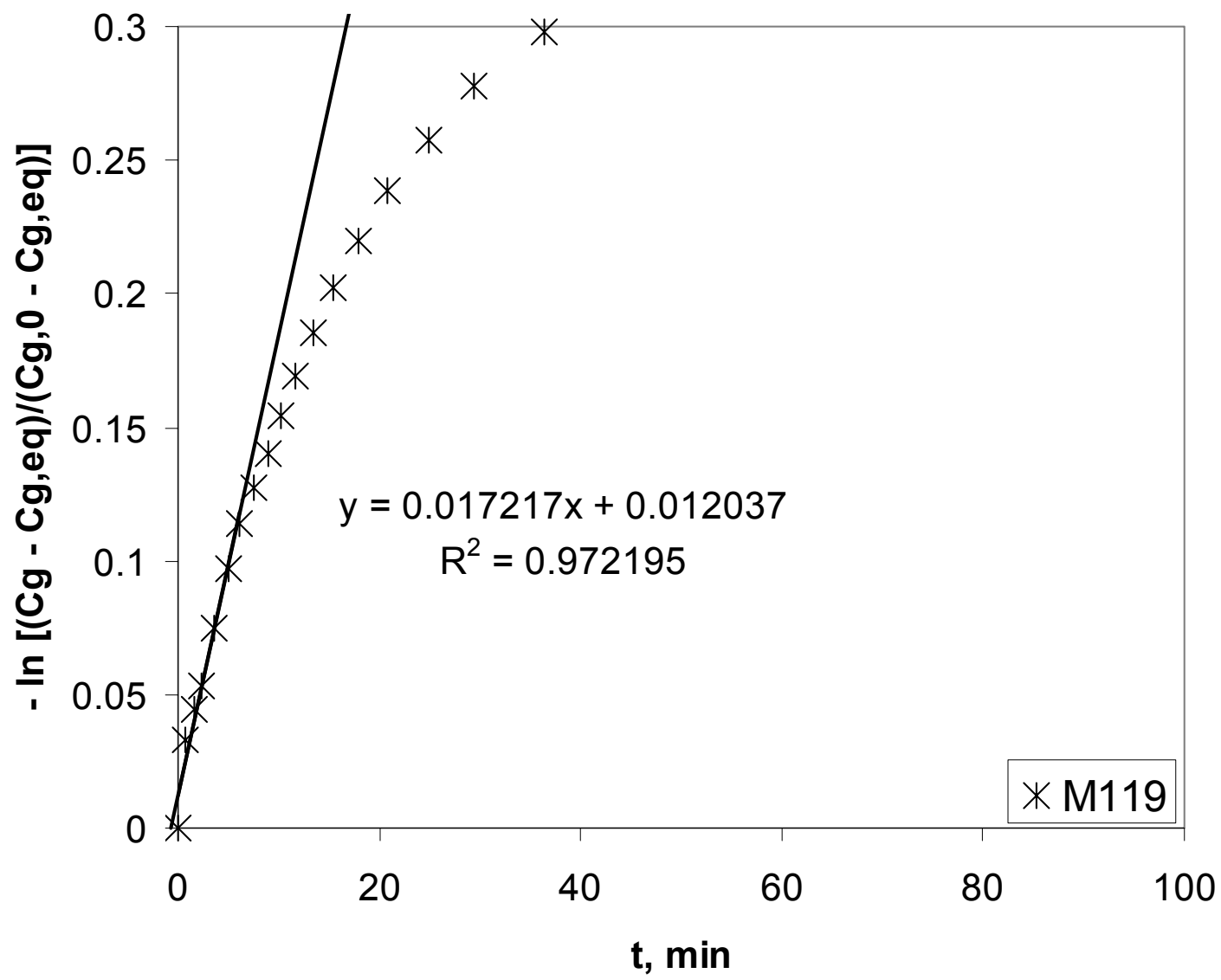

Figure 5.22. Evaluation of the MGH formation experiment Run M119 with initial pressure 49.28 bar, $T=273.5 \mathrm{~K}, d_{p}=510 \mu \mathrm{m}$, and $S$ $=0.30$. 


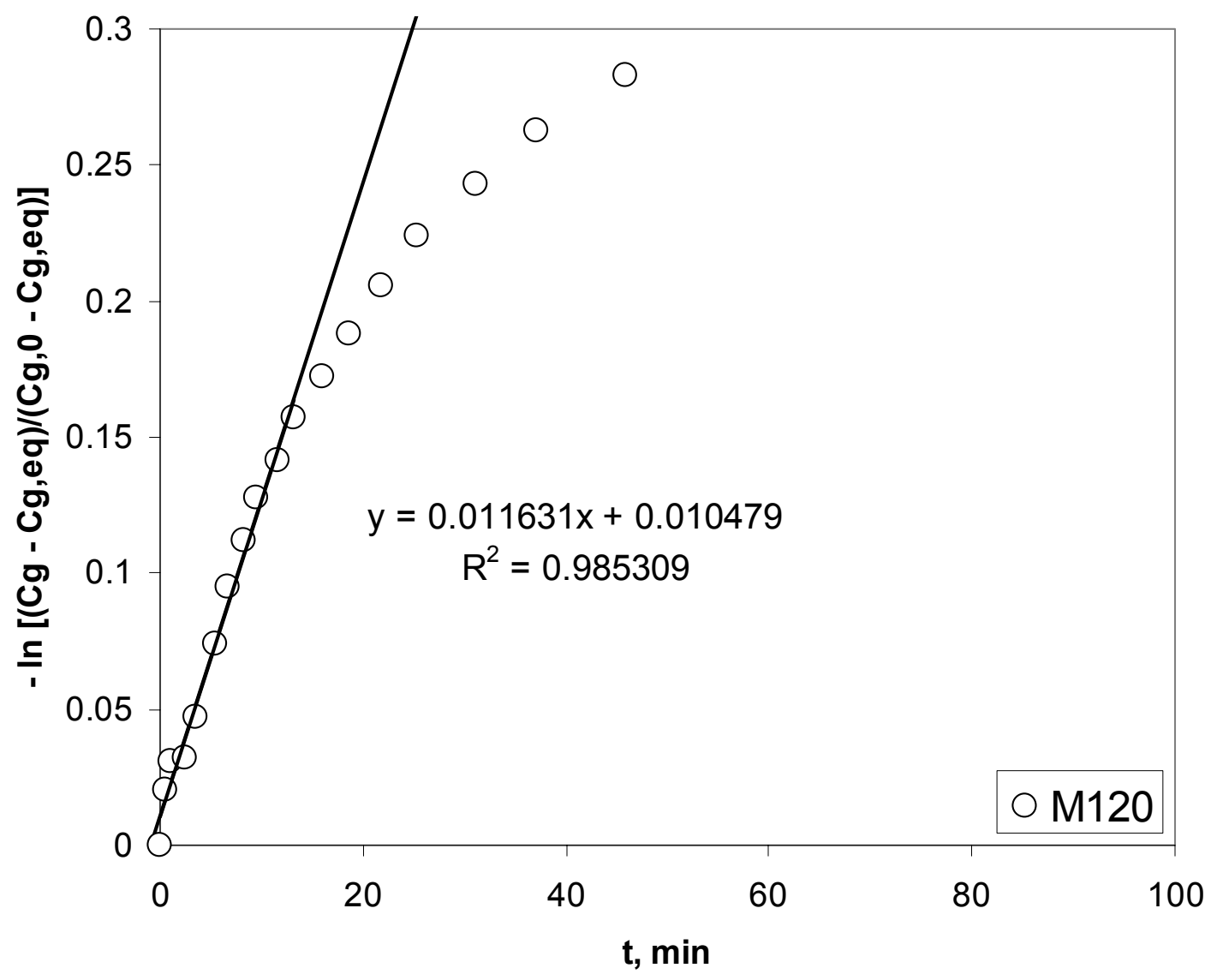

Figure 5.23. Evaluation of the MGH formation experiment Run M120 with initial pressure 49.28 bar, $T=273.5 \mathrm{~K}, d_{p}=510 \mu \mathrm{m}$, and $S$ $=0.30$. 
TABLE 5.1

Evaluation of $K$ for MGH Formation Experimental Data

\begin{tabular}{|l|c|c|c|c|c|}
\hline Run & $\left(-\frac{K A}{H V_{g}}\right), \mathrm{min}^{-1}$ & & $V_{g}, \mathrm{~mL}$ & $H$ & $K \times 10^{6}, \mathrm{~m} / \mathrm{s}$ \\
\hline M102 & 0.026019 & 0.9830 & 209.475 & 18.0 & 1.66 \\
\hline M103 & 0.026413 & 0.9830 & 209.475 & 18.0 & 1.69 \\
\hline M104 & 0.025136 & 0.9830 & 209.475 & 18.0 & 1.61 \\
\hline M105 & 0.022262 & 0.9830 & 209.475 & 18.0 & 1.42 \\
\hline M106 & 0.028426 & 0.9830 & 209.475 & 18.0 & 1.82 \\
\hline M107 & 0.030984 & 0.9830 & 209.475 & 18.0 & 1.98 \\
\hline M108 & 0.029611 & 0.9830 & 209.475 & 18.0 & 1.89 \\
\hline M109 & 0.038120 & 0.9830 & 209.475 & 18.0 & 2.43 \\
\hline M110 & 0.036303 & 0.9830 & 209.475 & 18.0 & 2.32 \\
\hline M111 & 0.025616 & 0.9830 & 209.475 & 18.0 & 1.64 \\
\hline M112 & 0.030747 & 0.9830 & 209.475 & 18.0 & 1.96 \\
\hline M113 & 0.030073 & 0.9830 & 209.475 & 18.0 & 1.92 \\
\hline M114 & 0.033575 & 0.9830 & 209.475 & 18.0 & 2.14 \\
\hline M115 & 0.017226 & 0.4327 & 205.8 & 18.0 & 2.46 \\
\hline M116 & 0.010273 & 0.4327 & 205.8 & 18.0 & 1.46 \\
\hline M117 & 0.011837 & 0.4327 & 205.8 & 18.0 & 1.69 \\
\hline M118 & 0.012385 & 0.4327 & 205.8 & 18.0 & 1.77 \\
\hline M119 & 0.017217 & 0.4327 & 205.8 & 18.0 & 2.45 \\
\hline M120 & 0.011631 & 0.4327 & 205.8 & 18.0 & 1.66 \\
\hline
\end{tabular}


The same calculations were done for data of PGH formation. Figures 5.24 and 5.25 show the raw experimental data. We should mention here that the experiments that involved the formation of PGH seeds, i.e. some induction periods, are not presented in the figures. The reason for not including PGH seeds formation experiments in the analysis of $K$ is the lack of data obtained in such experiments. It was not easy to predict when the growth period started in the first cycle of experiments. It was due to the stochastic nature of NGH nucleation. Because monitoring of pressure and temperature of reaction was done manually, we let the reaction to finish for the first cycle and then started data collection in the next cycles.

Furthermore, PGH formation rate constant $(K)$ was evaluated based on the model, as shown in Figures 5.26 to 5.31. The results are presented in Table 5.2.

TABLE 5.2

Evaluation of $K$ for PGH Formation Experimental Data

\begin{tabular}{|c|c|c|c|c|c|}
\hline Run & $\left(-\frac{K A}{H V_{g}}\right), \mathrm{min}^{-1}$ & $A, \mathrm{~m}^{2}$ & $V_{g}, \mathrm{~mL}$ & $H$ & $K \times 10^{6}, \mathrm{~m} / \mathrm{s}$ \\
\hline P101 & 0.034553 & 0.6028 & 228.585 & 10.8 & 2.36 \\
\hline P102 & 0.026454 & 0.6028 & 228.585 & 10.8 & 1.81 \\
\hline P103 & 0.037758 & 0.6028 & 228.585 & 10.8 & 2.58 \\
\hline P106 & 0.022053 & 0.4925 & 263.13 & 10.8 & 2.12 \\
\hline P107 & 0.031052 & 0.4925 & 263.13 & 10.8 & 2.99 \\
\hline P108 & 0.016779 & 0.4925 & 263.13 & 10.8 & 1.61 \\
\hline
\end{tabular}




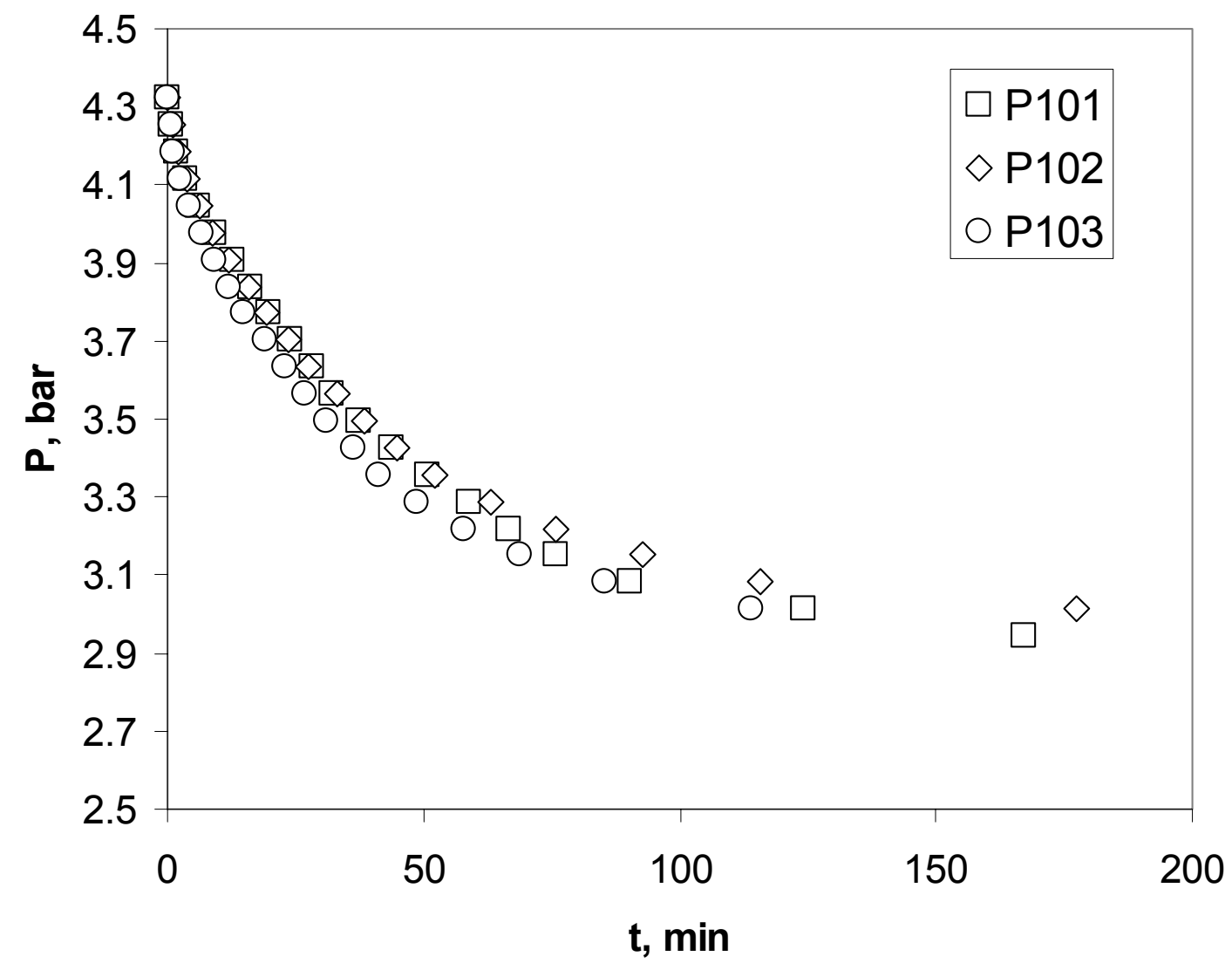

Figure 5.24. Raw data of PGH formation experiments for initial pressure $4.32 \mathrm{bar}, T=273.5 \mathrm{~K}, d_{p}=240 \mu \mathrm{m}$, and $S=0.30$. 


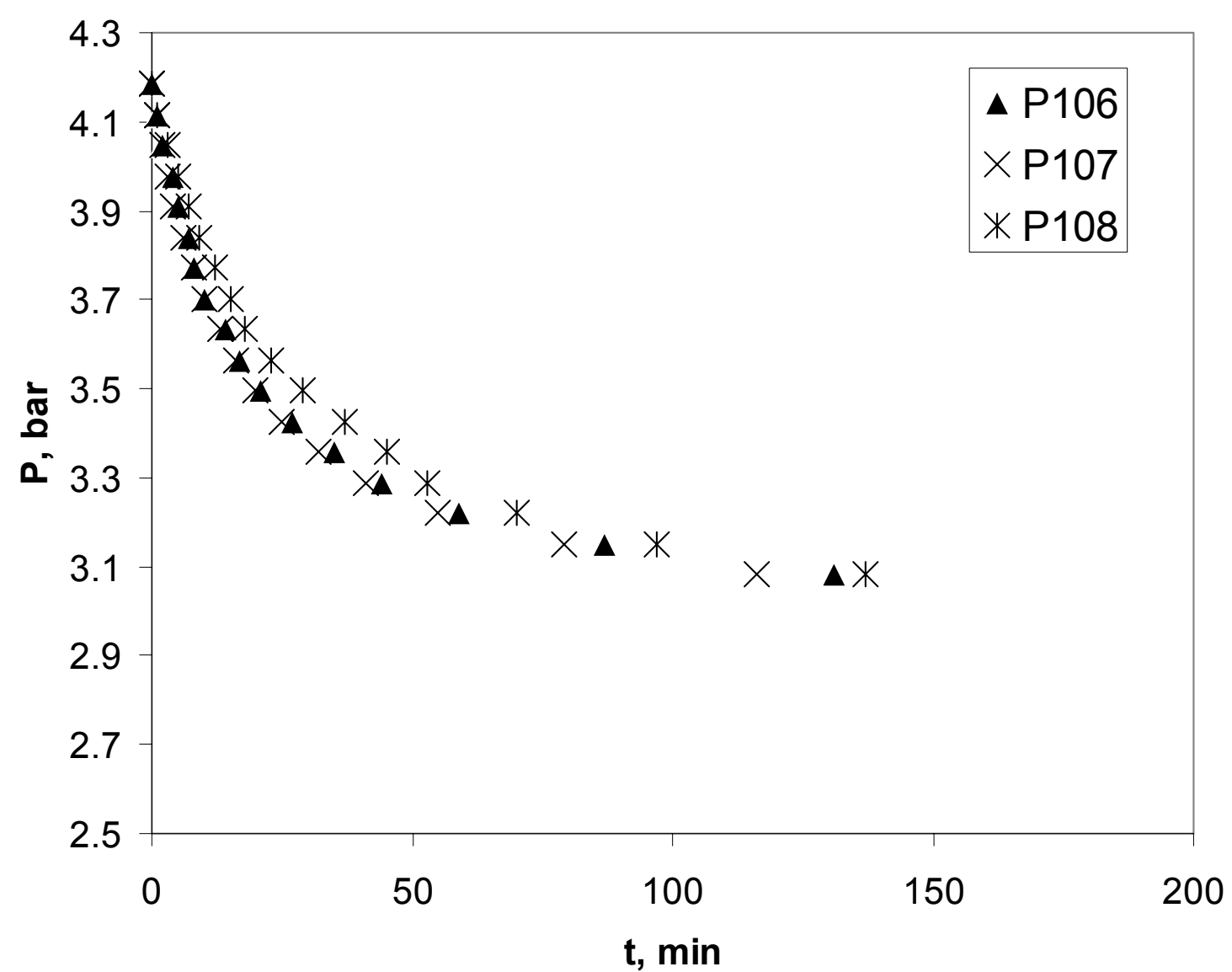

Figure 5.25. Raw data of PGH formation experiments for initial pressure $4.18 \mathrm{bar}, T=273.5 \mathrm{~K}, d_{p}=240 \mu \mathrm{m}$, and $S=0.30$. 


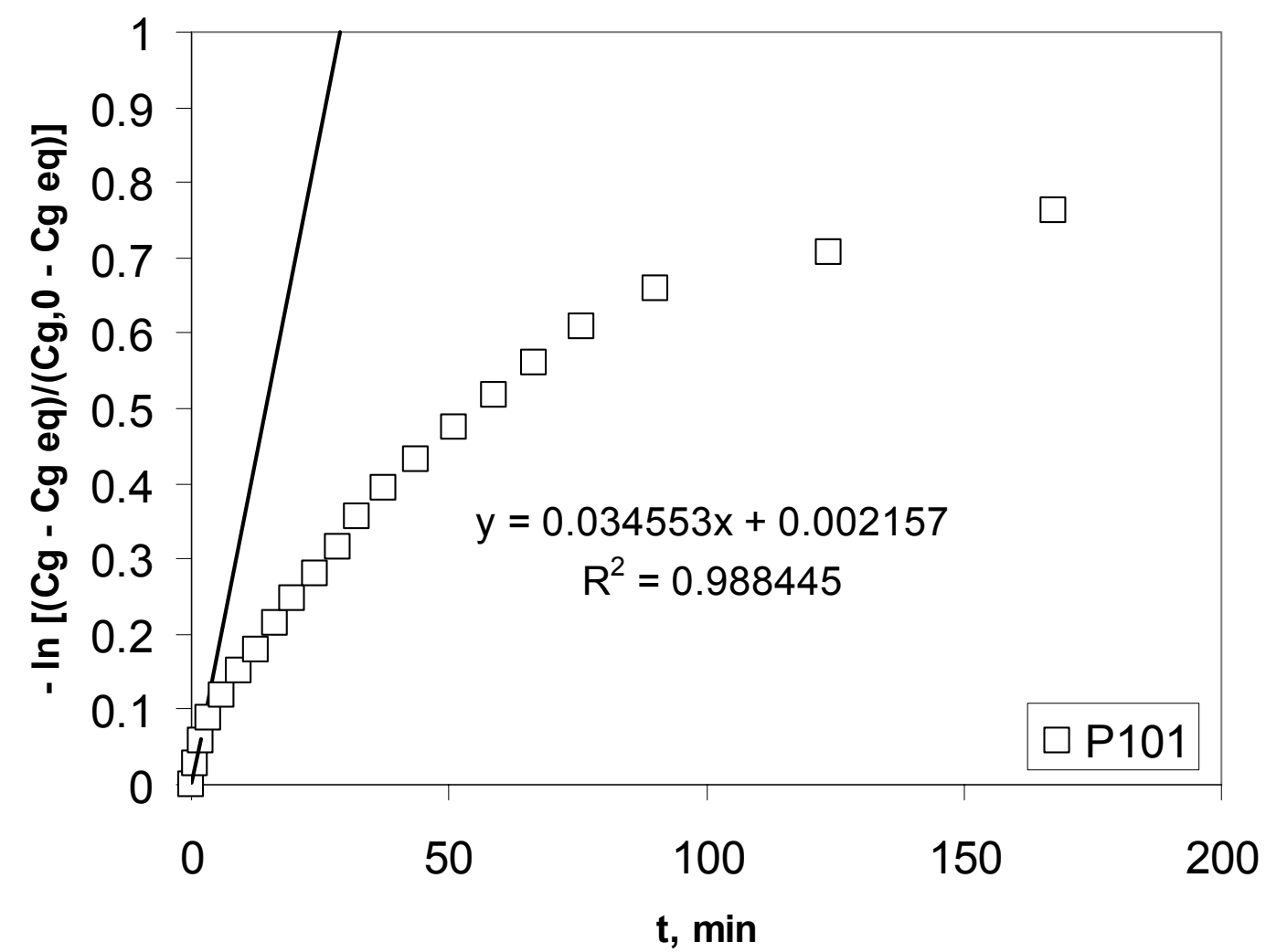

Figure 5.26. Evaluation of the PGH formation experiment Run P101 with initial pressure 4.32 bar, $T=273.5 \mathrm{~K}, d_{p}=240 \mu \mathrm{m}$, and $S=$ 0.30 . 


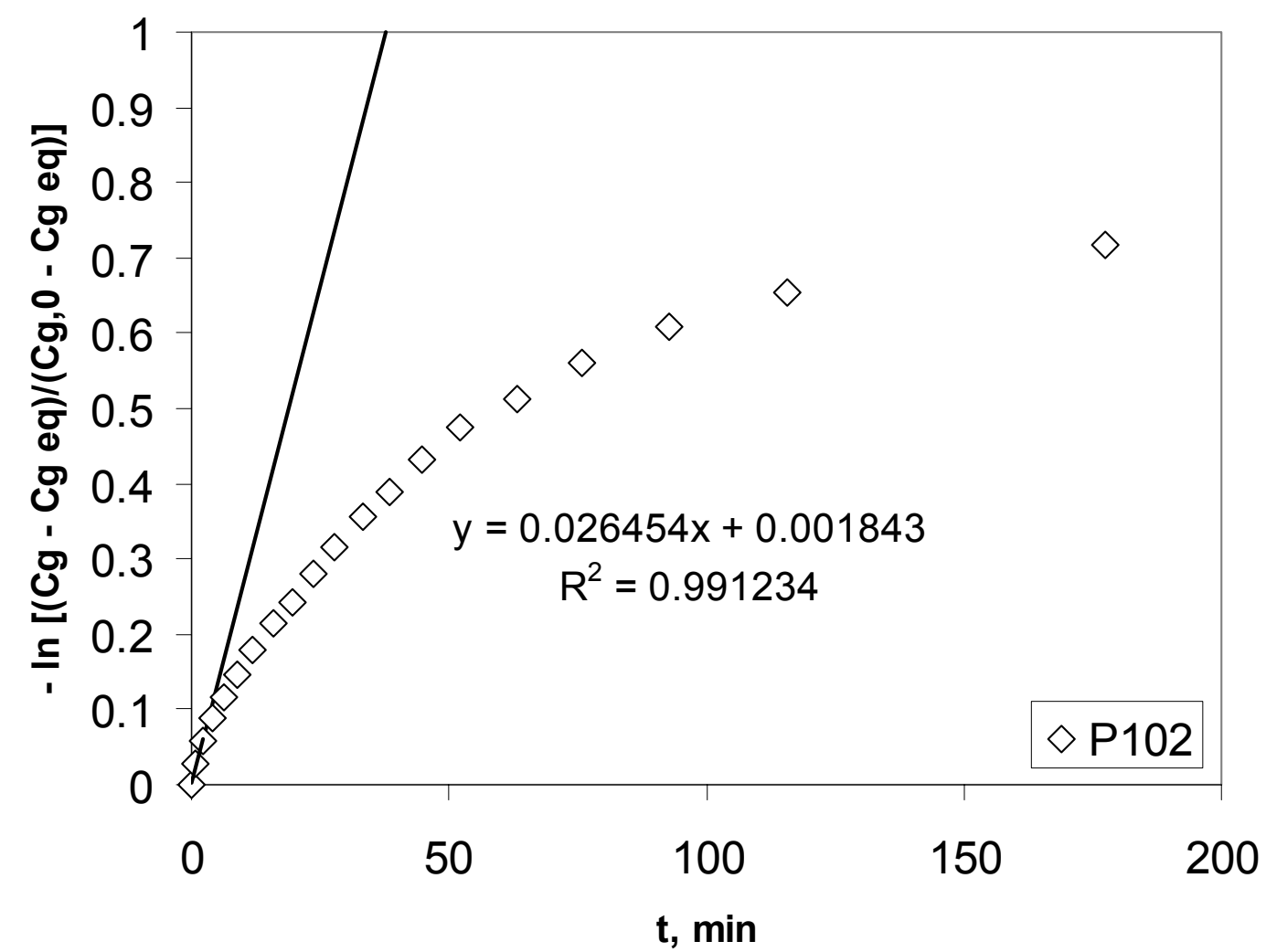

Figure 5.27. Evaluation of the PGH formation experiment Run P102 with initial pressure 4.32 bar, $T=273.5 \mathrm{~K}, d_{p}=240 \mu \mathrm{m}$, and $S=$ 0.30 . 


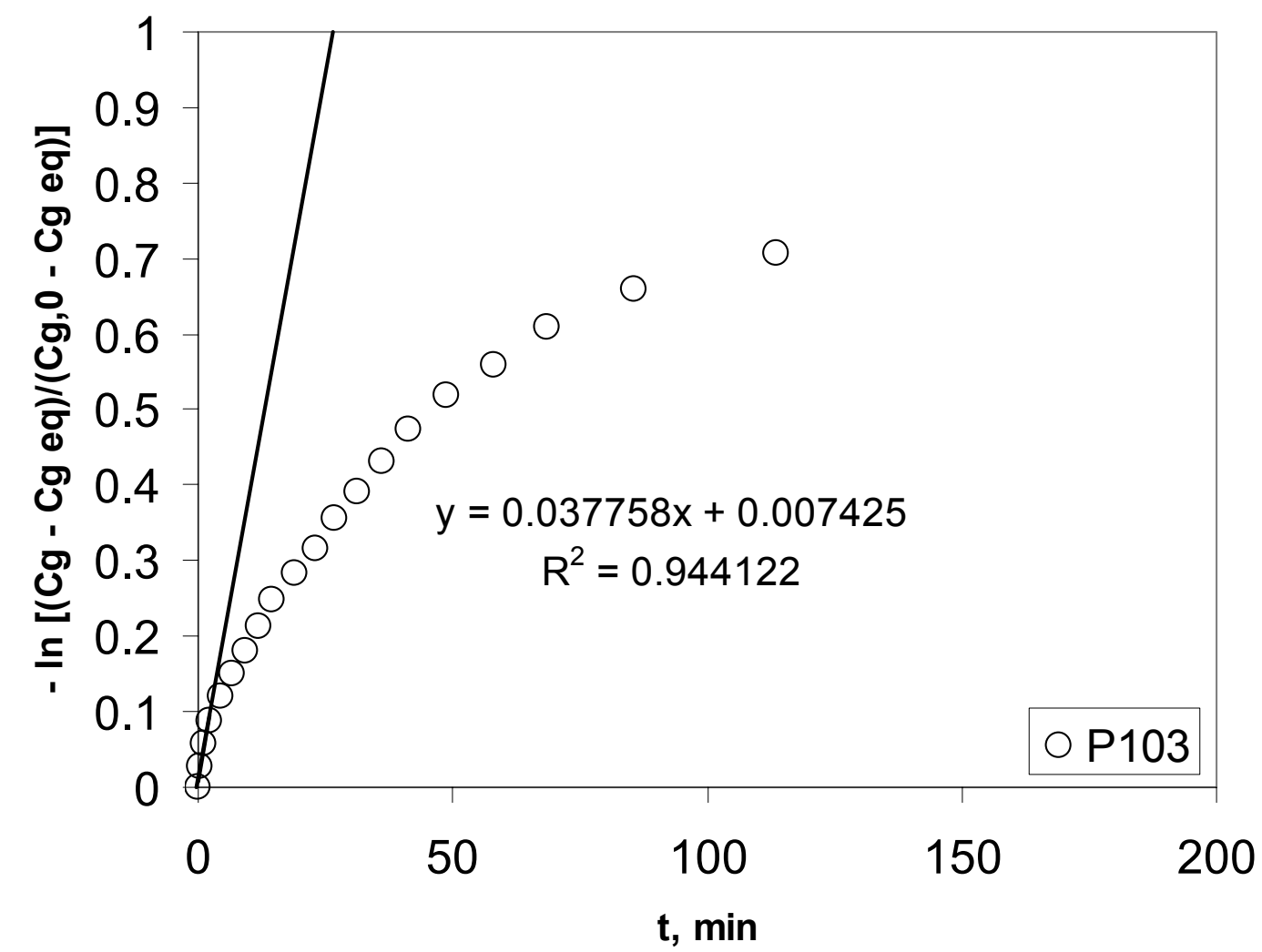

Figure 5.28. Evaluation of the PGH formation experiment Run P103 with initial pressure 4.32 bar, $T=273.5 \mathrm{~K}, d_{p}=240 \mu \mathrm{m}$, and $S=$ 0.30 . 


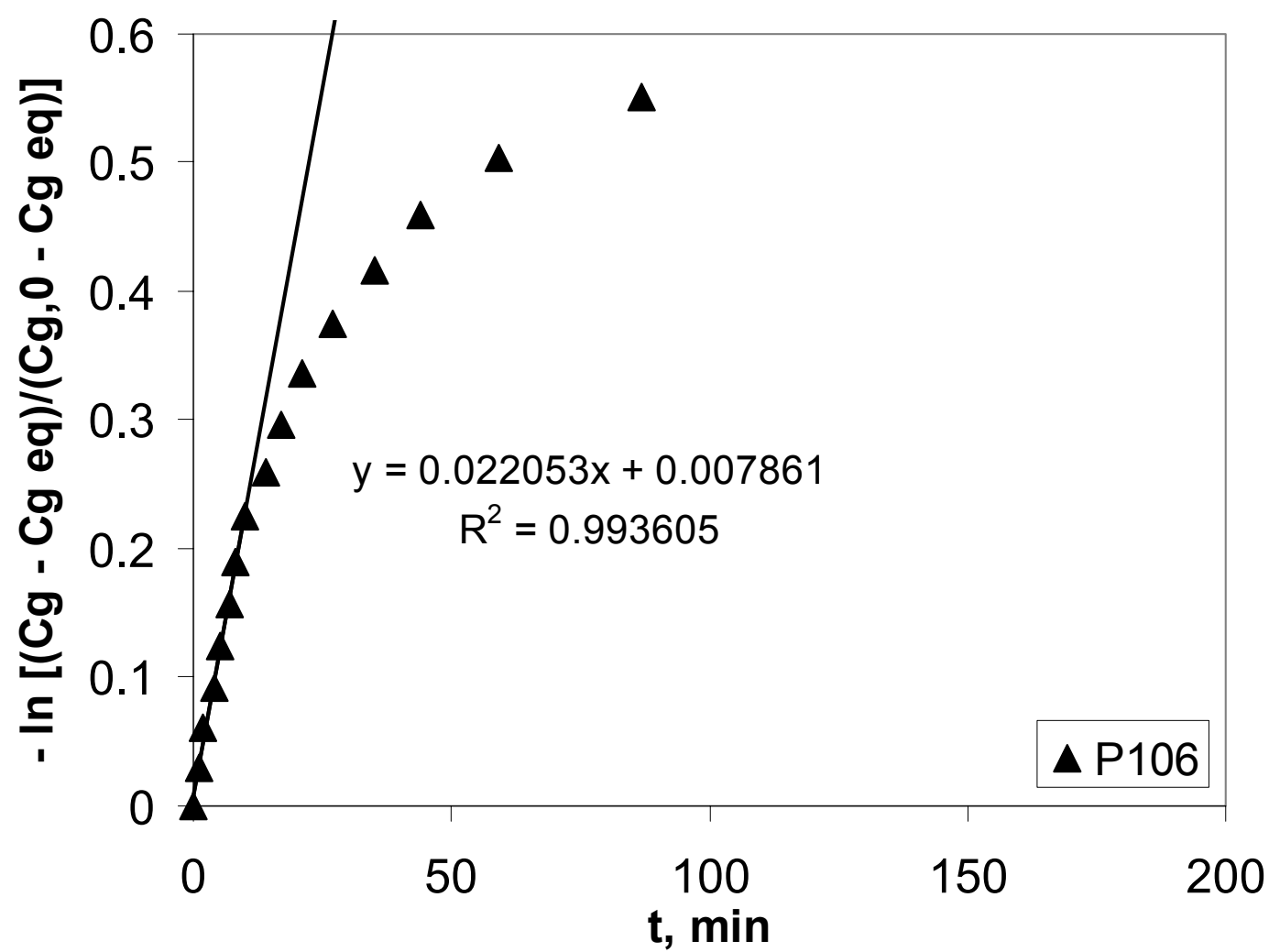

Figure 5.29. Evaluation of the PGH formation experiment Run P106 with initial pressure 4.18 bar, $T=273.5 \mathrm{~K}, d_{p}=240 \mu \mathrm{m}$, and $S=$ 0.30 . 


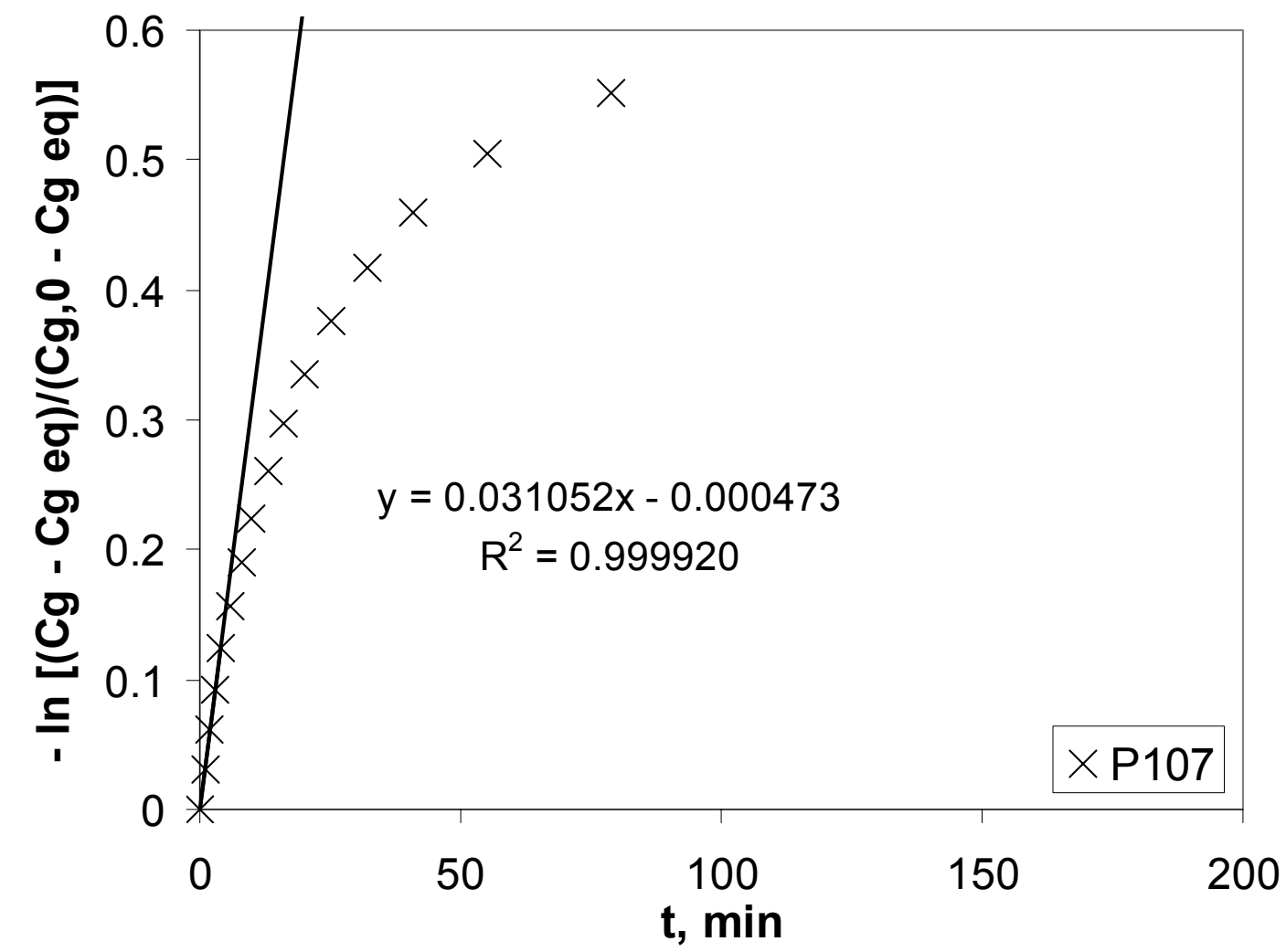

Figure 5.30. Evaluation of the PGH formation experiment Run P107 with initial pressure 4.18 bar, $T=273.5 \mathrm{~K}, d_{p}=240 \mu \mathrm{m}$, and $S=$ 0.30 . 


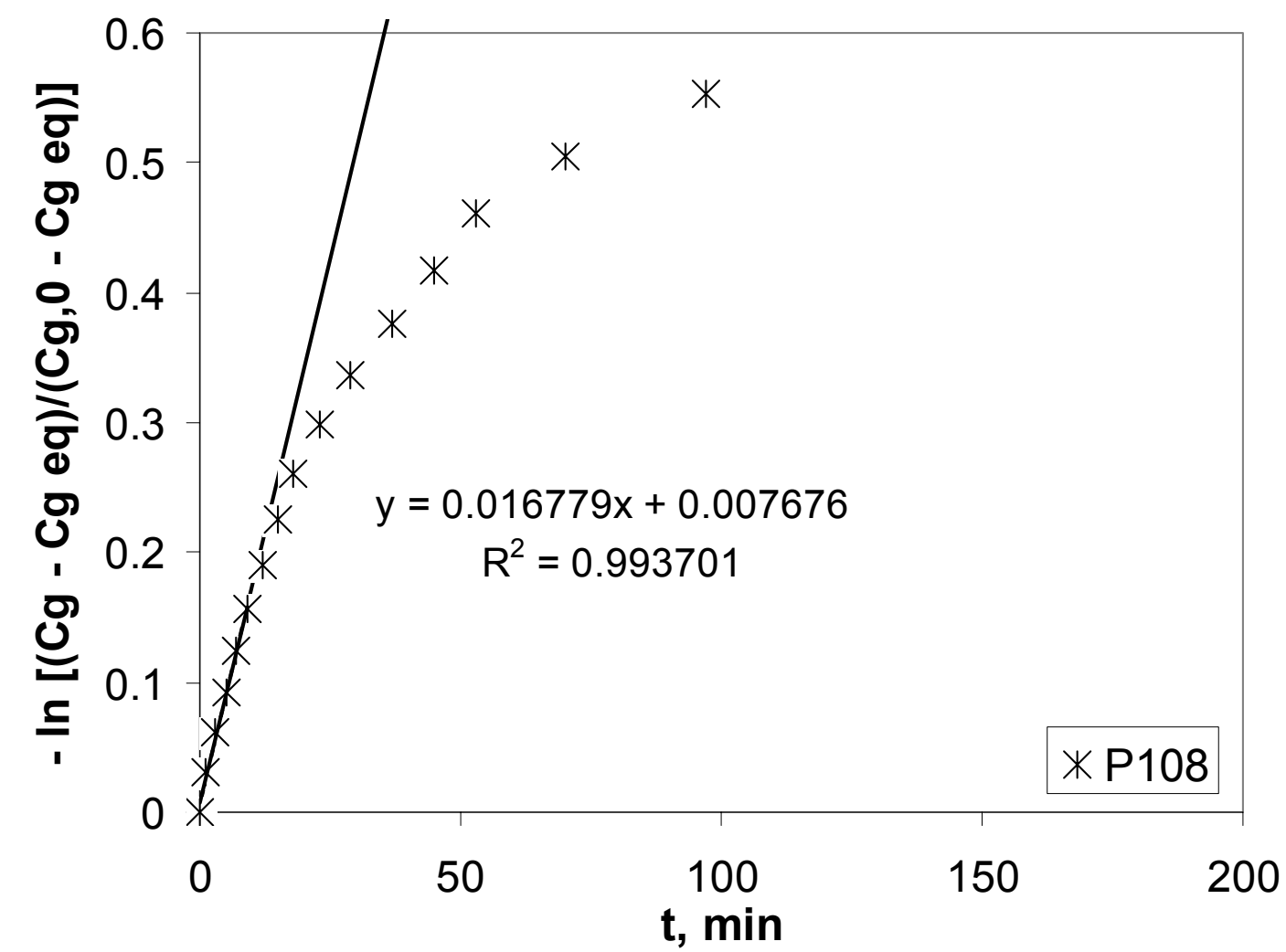

Figure 5.31. Evaluation of the PGH formation experiment Run P108 with initial pressure 4.18 bar, $T=273.5 \mathrm{~K}, d_{p}=240 \mu \mathrm{m}$, and $S=$ 0.30 . 
From the data of experiments, we can also calculate the apparent hydration number. As we discussed in Chapter 2, the hydration number is defined as the ratio of the number of water molecules to the number of NG molecules in the NGH. Hence, the apparent hydration number $\left(n_{w, a p p .}\right)$ can be calculated from the total moles of NG consumed at the end of the reaction and the total moles of water added to the reactor initially.

$$
n_{w, \text { app }}=\frac{W_{H 2 O}}{M_{H 2 O}\left(C_{g, 0}-C_{g, \text { end }}\right) V_{g}}
$$

Here, $C_{g, \text { end }}$ is the concentration of the NG in the gas phase at the end of reaction; $C_{g, 0}$ is the initial concentration of the NG in the gas phase; $V_{g}$ is volume of the gas phase; $W_{H 2 O}$ is water weight; and $M_{H 2 O}$ is molecular weight of water. To get $C_{g, \text { end }}$, the reacting system was left overnight until no more pressure drop was observed. Data of $C_{g \text {,end }}$ are not presented in the figures.

Furthermore, the amount of water reacted at a known $C_{g}$ can be predicted using the $n_{w, a p p .}$ As an approximation, moles of water converted to $N G H=n_{w, a p p .}\left(C_{g, 0}-C_{g}\right) V_{g}$

Then, conversion of water $\left(X_{\mathrm{H} 2 \mathrm{O}}\right)$ can be calculated with the assumption that at the end of reaction, all water was consumed to $\mathrm{NGH}$.

Summary of data of $K$, together with $n_{w, a p p}$, and $X_{H 2 O}$ when experimental data started to depart from the linear line of the model are presented in Tables 5.3 and 5.4. 
TABLE 5.3

$K$ of MGH in the Growth Period with the Apparent Hydration Number $\left(n_{w, a p p}\right)$ and the

Water Conversion when the Data Depart from the Linear Line of the Model $\left(X_{H 2 O}\right)$

\begin{tabular}{|c|c|c|c|c|c|}
\hline Run & Initial P, bar & $d_{p}, \mu \mathrm{m}$ & $K \times 10^{6}, \mathrm{~m} / \mathrm{s}$ & $n_{w, a p p}$ & $X_{H 2 O}$ \\
\hline M102 & 49.28 & 240 & 1.66 & 7.35 & 0.4499 \\
\hline M103 & 49.28 & 240 & 1.69 & 8.22 & 0.2528 \\
\hline M104 & 49.28 & 240 & 1.61 & 8.14 & 0.4773 \\
\hline M105 & 49.28 & 240 & 1.42 & 7.35 & 0.5815 \\
\hline M106 & 49.28 & 240 & 1.82 & 7.51 & 0.3984 \\
\hline M107 & 49.28 & 240 & 1.98 & 7.67 & 0.4919 \\
\hline M108 & 49.28 & 240 & 1.89 & 7.83 & 0.4810 \\
\hline M109 & 42.38 & 240 & 2.43 & 9.10 & 0.2702 \\
\hline M110 & 42.38 & 240 & 2.32 & 8.43 & 0.3811 \\
\hline M111 & 42.38 & 240 & 1.64 & 8.43 & 0.5325 \\
\hline M112 & 45.83 & 240 & 1.96 & 7.88 & 0.5497 \\
\hline M113 & 45.83 & 240 & 1.92 & 7.43 & 0.4926 \\
\hline M114 & 45.83 & 240 & 2.14 & 7.69 & 0.5076 \\
\hline M115 & 49.28 & 510 & 2.46 & 6.14 & 0.4517 \\
\hline M116 & 49.28 & 510 & 1.46 & 5.77 & 0.2367 \\
\hline M117 & 49.28 & 510 & 1.69 & 5.89 & 0.2686 \\
\hline M118 & 49.28 & 510 & 1.77 & 6.00 & 0.3440 \\
\hline M119 & 49.28 & 510 & 2.45 & 5.81 & 0.2743 \\
\hline M120 & 49.28 & 510 & 1.66 & 5.82 & 0.3647 \\
\hline & & & & & \\
\hline
\end{tabular}


TABLE 5.4

$K$ of PGH in the Growth Period with the Apparent Hydration Number $\left(n_{w, a p p}\right)$ and the Water Conversion when the Data Depart from the Linear Line of the Model $\left(X_{H 2 O}\right)$

\begin{tabular}{|c|c|c|c|c|c|}
\hline Run & Initial P, bar & $d_{p}, \mu \mathrm{m}$ & $K \times 10^{6}, \mathrm{~m} / \mathrm{s}$ & $n_{w, a p p}$ & $X_{H 2 O}$ \\
\hline P101 & 4.32 & 240 & 2.36 & 21.03 & 0.1016 \\
\hline P102 & 4.32 & 240 & 1.81 & 21.02 & 0.1016 \\
\hline P103 & 4.32 & 240 & 2.58 & 19.62 & 0.1420 \\
\hline P106 & 4.18 & 240 & 2.12 & 18.19 & 0.4185 \\
\hline P107 & 4.18 & 240 & 2.99 & 18.18 & 0.2432 \\
\hline P108 & 4.18 & 240 & 1.61 & 19.24 & 0.3207 \\
\hline
\end{tabular}

\subsection{Discussion}

Tables 5.3 and 5.4 show that the final ratio of moles of reacted NG to total moles of water in the bed (i.e. the apparent hydration number) varied from run to run, in the range of 5.77 to 9.10 for MGH, and in the range of 18.18 to 21.03 for PGH. These values are larger than the minimum possible hydration number which is 5.75 for MGH, and 17 for PGH (Makogon, 1997). It meant some cavities in the MGH/PGH crystals were not occupied by methane/propane molecules.

Figures 5.5 to 5.23 and 5.26 to 5.31 show the model was good only in the first minutes of the reactions, when the data started to depart from the linear lines of the model. To explain this phenomenon, we need to know where the MGH/PGH formed in 
the water bridges. There are at least three possible assumptions about the place where $\mathrm{MGH} / \mathrm{PGH}$ was formed in the reacting system.

The first possibility is MGH/PGH was formed right at the gas-water (1) interface where the concentration of the dissolved methane/propane was the highest. Because the density of $\mathrm{MGH} / \mathrm{PGH}$ (i.e. at $T=273 \mathrm{~K}, P=2.56 \mathrm{MPa}$, the density of $\mathrm{MGH}$ is 0.910 $\mathrm{g} / \mathrm{mL}$; while at $T=273 \mathrm{~K}, P=0.172 \mathrm{MPa}$, the density of PGH is $0.866 \mathrm{~g} / \mathrm{mL}$ (Makogon, 1997)) was smaller than the density of water, there was possibility of MGH/PGH crystal expansion during its formation that resulted in some cracking of the MGH/PGH crystals. The cracks created channels for methane/propane to access the water (1) for further reaction. The mechanism might involve the movement of water through the cracks by capillary force.

The second possibility is the reaction took place continuously everywhere in the whole water bridge. Due to the size of a single water bridge that was in the order of liquid film thickness (i.e. for $S=0.30$, the normal distances between the water bridge meniscus and the point of contact between two glass beads $\left(r_{2}\right)$ are $6.7891 \times 10^{-5}$ and $14.2743 \times 10^{-5}$ m for glass bead diameters 240 and $510 \mu \mathrm{m}$ respectively), the effect of gravity on the MGH/PGH seeds in such small system was negligible. As a result the concentration of MGH/PGH seeds was approximately homogeneous everywhere in the water bridges. It made possible for $\mathrm{MGH} / \mathrm{PGH}$ crystal growth to happen everywhere in the water bridges.

The third possibility is the reaction took place from the wetted surfaces of glass beads, growing in the direction of the gas-liquid interface. The formation of crystalline NGH normally requires a solid nucleation site (Lee et al., 1998). Thermodynamically, the foreign surfaces effectively lower the excess Gibbs free energy between NGH seeds and 
dissolved NG in the solution. As a result, the critical radius required for the NGH crystal growth lowers, which favors the NGH crystal growth (Sloan, 1998a). In our reacting system, the foreign surfaces could be provided by the wetted surfaces of glass beads. As a result, it was possible for NGH crystal to grow on the wetted surfaces of glass beads.

All the three possibilities have not been proved by direct observations due to the limitations of the experimental equipments. Furthermore, the departure of the data from the model might be explained as follows. If the first possibility about the place where $\mathrm{NGH}$ formed is correct, the departure of the data from the linear line of the model could be caused by the additional mass transfer resistance contributed by MGH/PGH layer cracks, besides the effective surface area of gas-water (1) interface decreased. Although the possibility of additional mass transfer resistance caused by MGH/PGH layers cracks might be correct, Equation (3.21) was still valid in the analysis of $K$ because each run started with no MGH/PGH layer resistance and analysis was done only in the first minutes of the run. On the other hand, if the second or the third possibility about the place where NGH formed is correct, the departure of the data from the linear line of the model might be caused by the decrease in surface area of contact between methane/propane (g) and water (1). In other words, the MGH/PGH layer covered partially the surface area of contact between methane/propane $(\mathrm{g})$ and water (1).

From Tables 5.3 and 5.4, we observe that in average, the data started to depart from the linear lines of the model at higher conversion of water in MGH formation than water conversion in PGH formation. We can not apply a similar comparison on conversion of methane/propane gas because not all runs started at the same initial concentration of methane/propane $\left(C_{g, 0}\right)$ and in all runs, methane/propane was in excess 
relative to water. Hence, we can not make direct comparisons among Figures 5.5 to 5.23 and 5.26 to 5.31. By the way, because higher conversion of water is related to larger amount of methane/propane consumed, we can say that the data started to depart from the linear lines of the model at higher consumption of methane than that of propane. This statement is even stronger with the fact that the ideal hydration number of MGH $(=5.75)$ is smaller than that of PGH $(=17)$. As we discussed previously in Chapter 2, the ideal hydration number is the ratio of moles of water to moles of NG in NGH in case of full occupancy of NGH crystal cavities by NG. The ideal hydration numbers shows that for 1 mole of water forming MGH, it takes 1/5.75 (=0.1739) moles of methane; while for 1 mole of water forming PGH, it takes only 1/17 $(=0.0588)$ moles of propane.

The possible explanation for the fact that the data started to depart from the linear lines of the model at higher consumption of methane than that of propane is as follows. Methane can occupy both types of cavities in the S-I type of MGH crystal, while propane can occupy only the larger cavities in the S-II type of PGH crystal. In the course of the reaction, both larger and smaller cavities were formed. While methane occupied both smaller and larger cavities of sI crystals, propane occupied only the larger cavities of sII crystals. As a result, for a certain amount of water had formed cavities, the amount of methane consumed in $\mathrm{MGH}$ formation reaction were larger than the amount of propane consumed in PGH formation. Because MGH/PGH crystals formed could block the methane/propane (g) - water (1) contact, it makes sense that PGH formation reaction data departed from the model at a lower consumption of propane than consumption of methane in the case of MGH formation reaction data. This statement that concerns about methane/propane consumption is more appropriate than the statement that concerns about 
water conversion, because our model relates $\left(\frac{C_{g}-C_{g}^{e q}}{C_{g, 0}-C_{g}^{e q}}\right)$ with $t$, not water conversion with $t$.

From Tables 5.3 and 5.4, we can see that the values of $K$ obtained from series of MGH/PGH formation experiments are approximately constant. Analysis of variance on the data ${ }^{1}$ gave the result that for $95 \%$ or larger level of confidence, there was no significant difference among $K$ values of different initial pressure. It meant initial pressure did not affect $K$. Analysis of variance on data of $K$ of different glass bead sizes also showed no significance difference among the $K$ values. It meant glass bead size did not affect $K$ either. It proved that mass transfer resistance in the gas phase along the pores of the bed of glass beads was negligible.

Furthermore, for $95 \%$ confidence interval, the data gave the value of $K=(1.89 \pm$ $0.16) \times 10^{-6} \mathrm{~m} / \mathrm{s}$ for MGH formation and $K=(2.25 \pm 0.53) \times 10^{-6} \mathrm{~m} / \mathrm{s}$ for PGH formation. Although the difference between $K$ of $\mathrm{MGH}$ formation and $K$ of $\mathrm{PGH}$ formation might be insignificant, these values suggested that $K$ of $\mathrm{MGH}$ formation is smaller than $K$ of PGH formation. In other words, $\mathrm{MGH}$ formation reaction is slower than $\mathrm{PGH}$ formation reaction. It is somewhat like what we expected. The explanation is as follows. According to the cluster nucleation theory (Sloan, 1998a), water molecules form labile clusters around dissolved NG molecules. The number of water molecules in each cluster is 20 for methane (in form of pentagonal dodecahedron $5^{12}$ cavity) and 28 for propane (in form of hexakaidecahedron $5^{12} 6^{4}$ cavity). Clusters of dissolved methane/propane combine to form unit cells of MGH/PGH crystal. One unit cell of MGH crystal consists of two $5^{12}$ cavities

\footnotetext{
${ }^{1}$ See Appendix E for analysis of variance on data of $K$.
} 
and six $5^{12} 6^{2}$ cavities. Because we used high purity methane, there was only one kind of clusters in the system, i.e. $5^{12}$ clusters. To form MGH crystals, some $5^{12}$ clusters needed to be transformed into $5^{12} 6^{2}$ clusters, by breaking and making hydrogen bonds among the water molecules. This transformation created an activation barrier. Similar phenomenon happens in PGH formation. One unit cell of PGH crystal consists of sixteen $5^{12}$ cavities and eight $5^{12} 6^{4}$ cavities. Because we used high purity propane, there was only one kind of clusters in the system, i.e. $5^{12} 6^{4}$ clusters. To form PGH crystals, some $5^{12} 6^{4}$ clusters needed to be transformed into $5^{12}$ clusters, by breaking and making hydrogen bonds among the water molecules. This transformation also created an activation barrier. The activation barrier is expected larger in MGH formation than that in PGH formation because of the following two reasons. First, in MGH crystals, the $5^{12} 6^{2}$ cavities outnumber $5^{12}$ cavities by a factor of $6 / 2(=3)$; while in PGH crystals, the $5^{12}$ cavities outnumber $5^{12} 6^{4}$ cavities only by a factor of $16 / 8(=2<3)$. Hence, more new clusters are needed in MGH formation than in PGH formation per unit original cluster. Second, the new $5^{12} 6^{2}$ clusters in MGH formation have more hydrogen bonds to be formed than the new $5^{12}$ clusters in PGH formation. As a result, MGH formation reaction should be slower than PGH formation reaction, as we observed.

In their experiments of MGH formation, Englezos et al. (1987a) got $K^{*}=0.65 \mathrm{x}$ $10^{-5} \mathrm{~mol} / \mathrm{m}^{2} \cdot \mathrm{s} \cdot \mathrm{MPa}$ at $274 \mathrm{~K}$, where $K^{*}$ is the reaction rate constant of MGH formation as defined by the following equation ${ }^{2}$.

$$
\left(\frac{d n}{d t}\right)_{p}=K^{*} A_{p}\left(f-f_{e q}\right)
$$

with

\footnotetext{
${ }^{2}$ See Appendix B for derivation of Englezos et al.’s model.
} 


$$
c=\frac{c_{w 0} f}{H^{*}}
$$

Equation (5.4) can be substituted into Equation (5.3) to get Equation (5.5).

$$
\left(\frac{d n}{d t}\right)_{p}=K^{*} A_{p} \frac{H^{*}}{c_{w 0}}\left(c-c_{e q}\right)
$$

To get the same form as Equation (3.18), we use Equation (3.17). The result is Equation (5.6).

$$
\left(\frac{d n}{d t}\right)_{p}=K^{*} A_{p} \frac{H^{*}}{H c_{w 0}}\left(C_{g}-C_{g}^{e q}\right)
$$

According to Equation (3.26),

$$
\frac{H^{*}}{H c_{w 0}}=\frac{H^{*} M_{H 2 O}}{H \rho_{w}}=z R T
$$

Equation (5.7) is substituted into Equation (5.6) to get Equation (5.8).

$$
\left(\frac{d n}{d t}\right)_{p}=K^{*}{ }_{z R T A_{p}}\left(C_{g}-C_{g}^{e q}\right)
$$

We should mention here that Equation (5.8) contains assumption that mass transfer of dissolved NG from gas-water (1) interface to the bulk of water (1) is not controlling, so it is in equilibrium. Furthermore, if we compare Equation (3.18) with Equation (5.8), we obtain the following relation.

$$
K=K * z T H
$$

Englezos et al. did their experiments at methane pressure in the range of $3.29-7.60 \mathrm{MPa}$ for the temperature of reaction $274 \mathrm{~K}$. The values of methane compressibility factor at these pressure and temperature are greater than 0.8 , which are close enough to 1 (ideal gas) (Perry and Green, 1985). If we assume methane is an ideal gas, then $z=1$. 
Furthermore, we use $H=18.0$, the value that we used in our data analysis. With $R=$ $8.3144 \times 10^{-6} \mathrm{MPa} \cdot \mathrm{m}^{3} / \mathrm{mol} \cdot \mathrm{K}$ and $T=274 \mathrm{~K}$, we obtain $K=2.67 \times 10^{-7} \mathrm{~m} / \mathrm{s}$. This value is about 7 times smaller than our $K$ value.

Englezos et al. claimed that their result represented the intrinsic reaction rate of MGH formation. Unfortunately, their experiments showed the influence of stirring rate on $K^{*}$ as shown in Figure 5.32. On their discussion in their paper, they explained it as there was a change from intrinsic reaction control to mass transfer control with decreasing stirring rate. According to Skovborg and Rasmussen (1994), at $274 \mathrm{~K}$ the lower limit of mass transfer coefficient in the water (1) film around the MGH particles of critical crystal size is approximately $1.0 \mathrm{~mol} / \mathrm{m}^{2} \cdot \mathrm{s} \cdot \mathrm{MPa}$, which is much larger than the observed $K^{*}\left(=0.65 \times 10^{-5} \mathrm{~mol} / \mathrm{m}^{2} \cdot \mathrm{s} \cdot \mathrm{MPa}\right)^{3}$. It means the decrease in stirring rate should not change the reaction from the intrinsic reaction controlled regime to mass transfer controlled regime. Because the intrinsic reaction rate is still controlling with decreasing stirring rate, it means Englezos et al.'s data showed the dependence of their intrinsic reaction rate constant on stirring rate. It should not be the case for the intrinsic reaction rate, which must be independent of stirring rate.

\footnotetext{
${ }^{3}$ If we take into account the Malegaonkar et al.'s modification, $K^{*}=3.1 \times 10^{-5} \mathrm{~mol} / \mathrm{m} 2 \cdot \mathrm{s} \cdot \mathrm{MPa}$. The modified $K^{*}$ is still much smaller than $1.0 \mathrm{~mol} / \mathrm{m}^{2} \cdot \mathrm{s} \cdot \mathrm{MPa}$.
} 


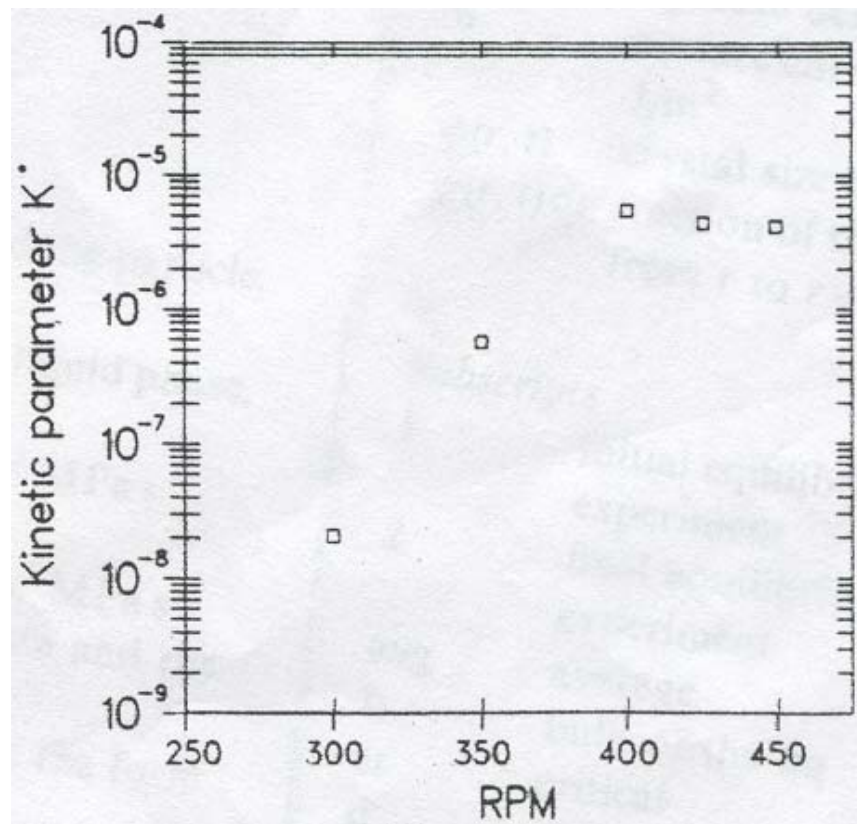

Figure 5.32. Effect of mixing on $K^{*}$ of Englezos et al.'s experiments (Figure 13 in Englezos et al.'s paper (1987a)).

Skovborg and Rasmussen (1994) simplified Englezos et al.'s model to take into account mass transfer resistance only, and they got the following equation.

$$
\frac{d n}{d t}=k_{L} A_{(g-l)^{c} c_{w 0}}\left(x_{i n t}-x_{b}\right)
$$

Using their mass transfer limited model, Skovborg and Rasmussen got $K=k_{L}=4.076 \mathrm{x}$ $10^{-5} \mathrm{~m} / \mathrm{s}$ for MGH formation. The value is in the order of $10^{-5} \mathrm{~m} / \mathrm{s}$ as we expected. The value is about 22 times larger than our $K$ value. This result proved that although there was a possibility of the effect of MGH crystals/seeds on the value of $k_{L}$, but still in our experimental conditions, the intrinsic reaction rate resistance was still dominant relative to the mass transfer resistance.

Now if we compare the value of $K$ (i.e. $K=(1.89 \pm 0.16) \times 10^{-6} \mathrm{~m} / \mathrm{s}$ for MGH formation and $K=(2.25 \pm 0.53) \times 10^{-6} \mathrm{~m} / \mathrm{s}$ for PGH formation) to $k_{L}$ (approximately is 
equal to $10^{-5} \mathrm{~m} / \mathrm{s}$ ), we can conclude that the resistance caused by the intrinsic reaction gave the major contribution to the total resistance. Mathematically, because $K$ is much smaller than $k_{L}$, then $K^{-1}$ is much larger than $k_{L}^{-1}$. Hence based on Equation (3.16), the intrinsic reaction rate constant $\left(k_{r}\right)$ was practically represented by the observed $K$.

In consequence of the negligible mass transfer resistance in the water liquid film relative to the intrinsic reaction resistance, the concentration profile of dissolved NG in the water liquid film as shown in Figure 3.5 becomes flat. In other words, $C_{i}$ is equal to $C^{*}$. Furthermore, based on the experimental data and calculation results, we can summarize the justification for the assumptions used in the derivation of the physical model of Equation (3.21) as follows.

1. The size of a single water bridge (i.e. $r_{2}$ were equal to $6.7891 \times 10^{-5}$ and $14.2743 \times$ $10^{-5} \mathrm{~m}$ for glass bead diameters 240 and $510 \mu \mathrm{m}$ respectively) was in the order of liquid film thickness whose typical value for many mass transfer processes is around $10^{-4} \mathrm{~m}$ (Cussler, 1997). Hence, we could consider the whole single water bridge as single liquid film. It is the justification for the assumption that there was only one water liquid film between gas-water (1) interface and the surface of MGH/PGH crystals.

2. The most probable site for the NGH formation was the wetted surfaces of glass beads. The reason is the glass bead surfaces effectively lower the excess Gibbs free energy between NGH seeds and dissolved NG in the solution. As a result, the critical radius required for the NGH crystal growth lowers, which favors the NGH crystal growth (Sloan, 1998a). As we concluded above, due to the negligible mass transfer resistance relative to the intrinsic reaction resistance, the concentration 
profile of the dissolved NG in the water liquid film was basically flat. There was only single water liquid film in each water bridge. As a result, the required high concentration of the dissolved NG could be found on the wetted surfaces of glass beads. Hence, it is very logical to conclude that NGH crystals grew from the wetted surfaces of glass beads. Because the surface area of the wetted surfaces of the glass beads was in the order of the surface area of gas-water (1) interface, then it is acceptable to assume that the total surface area of MGH/PGH crystals was approximately equal to the surface area of mass transfer, i.e. the surface area of the gas-water (1) interface. It is the justification for the assumption that the surface area of gas-water (1) interface was approximately equal to the total surface area of MGH/PGH crystals.

3. We used high purity methane/propane (99.90\%). Hence, no mass transfer resistance in the gas phase outside the bed. Furthermore, analysis of variance on the data showed insignificant effects of initial pressures and glass bead sizes on the reaction rate constants. It proved that mass transfer resistance in the gas phase along the pores of the bed of glass beads was negligible too. It is the justification for the assumption that the mass transfer resistance in the gas phase was negligible.

4. As we concluded above, the intrinsic reaction was slow, so mass transfer of dissolved NG in water liquid film was basically in equilibrium as shown by the flat concentration profile. Hence, the assumption that the mass transfer of dissolved NG in the water liquid film was in quasi steady state was not violated. 
5. Isothermal condition could be maintained during reaction in each experiment. It is the justification for the assumption that no heat transfer effects were involved.

6. In each run, there was an initial period in the growth period where the data fitted in the linear line of the model. It meant gradient of the line, i.e. $\left(-\frac{K A}{H V_{g}}\right)$, was also constant in the part of the growth period. Because the reaction was isothermal, $C_{g}^{e q}, K$, and $H$, which are functions of temperature only, must be constant. The volume of water inside the reactor was very small compare to the volume of the gas phase $\left(V_{g}\right)$ as shown in Table 4.1. (i.e. water volume was less than $5 \%$ of $V_{g}$ ). Hence, the change of water volume due to its phase change from water liquid into NGH was also negligible. Practically, $V_{g}$ was constant during the experiments. Because $K, H, V_{g}$, and $\left(-\frac{K A}{H V_{g}}\right)$ were constant, $A$ must be constant too. It is the justification for the assumption that $C_{g}^{e q}, K, A, H$, and $V_{g}$ were constant. 


\section{CHAPTER 6}

\section{CONCLUSION}

The clathrate kinetics of NGH formation in the heterogeneous gas-liquid-solid phase systems has recently attracted the wide attention of many industrial researchers, as the NGH may potentially become one of the largest alternative energy resources of the world in this century. Although several papers have been published on the clathrate kinetics of NGH formation reactions, the experimental works of reproducible intrinsic reaction rate constants of NGH formations have not been reported. It has been because the control of interfacial surface areas was experimentally difficult among the three phases involved in the reaction, i.e. NG in gas phase, water in liquid phase, and NGH in solid phase, during the progress of water-dissolved NG conversion into NGH.

In this research, the reaction rates of MGH and PGH formations were measured under a well-defined, reproducible gas-liquid-solid heterogeneous packing condition in a hand-made reactor. To obtain the intrinsic reaction rate constants of MGH and PGH formations, a specific reaction system was carefully prepared and designed by controlling the interfacial boundary area. Within the well-defined specific particle packing structure by using spherical, uniform-sized inorganic inert particles (i.e. glass beads), the surface area of the gas-liquid interface could be controlled and predicted. The well-defined packing structure gave reproducible interfacial boundary area of the heterogeneous reacting system.

Data showed that initial pressures and glass bead sizes used in the experiments did not affect the reaction rate constants. The physical model of MGH/PGH formation 
reaction gave good prediction until about maximum $58.15 \%$ conversion of water into MGH and about maximum $41.85 \%$ conversion of water into PGH, when the data started to depart from the linear lines of the physical model. It showed that the model gave good prediction until higher consumption of methane into MGH than the consumption propane into PGH. For $95 \%$ confidence interval, the data gave the value of $K=(1.89 \pm 0.16) \mathrm{x}$ $10^{-6} \mathrm{~m} / \mathrm{s}$ for MGH formation and $K=(2.25 \pm 0.53) \times 10^{-6} \mathrm{~m} / \mathrm{s}$ for PGH formation. Compared to $k_{L}$ (approximately is equal to $10^{-5} \mathrm{~m} / \mathrm{s}$ ), the values of $K$ are significantly smaller for both MGH and PGH formations. Hence, the intrinsic reaction rate constants $\left(k_{r}\right)$ of MGH and PGH formations were practically represented by the observed $K$. 


\section{REFERENCES}

Carroll, J.J., and A.E. Mather, "A Model for the Solubility of Light Hydrocarbons in Water and Aqueous Solutions of Alkanolamines", Chem. Eng. Sci., 52, 545-552 (1997).

Cussler, E.L., "Diffusion Mass Transfer in Fluid Systems”, 2 ed., Cambridge University Press, New York (1997) pp. 112, 334.

Dholabhai, P.D., N. Kalogerakis, and P.R. Bishnoi, "Kinetics of Methane Hydrate Formation in Aqueous Electrolyte Solutions”, Can. J. Chem. Eng., 71, 68-74 (1993).

Dowdy, S. and S. Wearden, "Statistics for Research", 2 ed.. Wiley-Interscience, New York (1991) pp. 283-297, 555,557-580.

Englezos, P., "Reviews. Clathrate Hydrates", Ind. Eng. Chem. Res., 32, 1251-1274 (1993).

Englezos, P., N. Kalogerakis, P.D. Dholabhai, and P.R. Bishnoi, "Kinetics of Formation of Methane and Ethane Gas Hydrates”, Chem. Eng. Sci., 42, 2647-2658 (1987a).

Englezos, P., N. Kalogerakis, P.D. Dholabhai, and P.R. Bishnoi, "Kinetics of Gas Hydrate Formation from Mixtures of Methane and Ethane”, Chem. Eng. Sci., 42, 2659-2666 (1987b).

Haughey, D.P. and G.S.G. Beveridge, "Structural Properties of Packed Beds - A Review", Can. J. Chem. Eng., 47, 130-140 (1969).

Jeffrey, G.A., "Hydrate Inclusion Compounds", in "Inclusion Compounds. Volume 1. Structural Aspects of Inclusion Compounds Formed by Inorganic and Organometallic 
Host Lattices", J.L. Atwood, J.E.D. Davies, and D.D. MacNicol, Eds., Academic Press Inc., Orlando, FL (1984) pp. 142-144.

Kane, S.G., T.W. Evans, P.L.T. Brian, and A.F. Sarofim, "Determination of the Kinetics of Secondary Nucleation in Batch Crystallizers", AIChE Journal, 20, 855-862 (1974)

Katz, D.L., D. Cornell, R. Kobayashi, F.H. Poettmann, J.A. Vary, J.R. Elenbaas, and C.F. Weinaug, "Handbook of Natural Gas Engineering”, McGraw-Hill, New York (1959) p. 802 .

Keen, B.A., "On the Moisture Relationships in an Ideal Soil”, Journ. Agric. Sci., 14, 170177 (1924).

Kono, H.O. and B. Budhijanto, "Modeling of Gas Hydrate Formation Processes by Controlling the Interfacial Boundary Surfaces", in "Proc. $4^{\text {th }}$ Int. Conf. on Gas Hydrates", 1, Yokohama, Japan, May 19-23, 2002 (2002) pp. 461-464.

Kono, H.O., S. Narasimhan, F. Song, and D.H. Smith, "Synthesis of Methane Gas Hydrate in Porous Sediments and Its Dissociation by Depressurizing", Powder Technology, 122, 239-246 (2002).

Lee, S.Y., E. McGregor, and G.D. Holder, "Experimental Study of Hydrate Crystal Growth from Methane, Carbon Dioxide, and Methane + Propane Mixtures”, Energy and Fuels, 12, 212-215 (1998).

Makogon, Y.F., "Hydrates of Natural Gas", translated by W.J. Cieslewics, PennWell Books, Tulsa, OK (1981) pp. 71-72.

Makogon, Y.F., "Hydrates of Hydrocarbons", PennWell Books, Tulsa, OK (1997) pp. XXI, 13-14, 30-31, 45, 95. 
Malegaonkar, M.B., P.D. Dholabhai, and P.R. Bishnoi, "Kinetics of Carbon Dioxide and Methane Hydrate Formation”, Can. J. Chem. Eng., 75, 1090-1099 (1997).

Perry, R.H. and D. Green, “Perry’s Chemical Engineers’ Handbook”, 6 ed., McGraw-Hill Book Co., Singapore (1985) pp. 3.63, 3.75, 3.103, 3.116, 19.29.

Skovborg, P., H.J. Ng, P. Rasmussen, and U. Mohn, "Measurement of Induction Times for the Formation of Methane and Ethane Gas Hydrates”, Chem. Eng. Sci., 48, 445453 (1993).

Skovborg, P. and P. Rasmussen, "A Mass Transport Limited Model for the Growth of Methane and Ethane Gas Hydrates”, Chem. Eng. Sci., 49, 1131-1143 (1994).

Sloan, E.D., Jr., “Clathrate Hydrates of Natural Gases”, 2 ed., Marcel Dekker, Inc., New York (1998a) pp. 12-13, 33, 53-55, 77-80, 86-91, 113.

Sloan, E.D., Jr., "Reviews. Gas Hydrates: Review of Physical/Chemical Properties", Energy \& Fuels, 12, 191-196 (1998b).

Smith, J.M., H.C. Van Ness, and M.M. Abbott, "Introduction to Chemical Engineering Thermodynamics", 5 ed., the McGraw-Hill Companies, Inc., New York (1996) pp. $87-90,635$.

Vysniauskas, A. and P.R. Bishnoi, “A Kinetic Study of Methane Hydrate Formation”, Chem. Eng. Sci., 38, 1061-1072 (1983).

Vysniauskas, A. and P.R. Bishnoi, "Kinetics of Ethane Hydrate Formation”, Chem. Eng. Sci., 40, 299-303 (1985). 


\begin{abstract}
APPENDICES
Appendix A is the part of the derivation of the water bridge dispersion model, which is our physical model of the NGH formations in our special packed bed reactor. Appendix B discusses Englezos et al's model and its modification; while Appendix C discusses Skovborg and Rasmussen's model. The two models are presented as the comparisons to our model. Englezos et al's experiments seemed to fail to obtain the reaction rate constants of $\mathrm{MGH}$ formations that were free from mass transfer effects, as shown by the effect of stirring rate on their $K$ values. On the other hand, the Skovborg and Rasmussen's model could not be used to determine the intrinsic reaction rate constants, due to its assumption that the transport of NG molecules from the gas-water (1) interface to the bulk of the liquid water phase is the rate determining step in the overall NGH formation process.

Appendix D provides the information on the method of glass bead bulk and true density measurements, together with the data of observations. Appendix E shows the statistical analysis on the data of $K$ obtained from the experiments.
\end{abstract}




\section{APPENDIX A}

\section{DERIVATIONS OF THE EQUATIONS OF THE SURFACE AREA AND THE VOLUME OF A SINGLE WATER BRIDGE}

The model of a single water bridge is shown in Figure A.1. The surface area of the single water bridge is the area of the surface of revolution of the water meniscus around the point of contact between the two spherical glass beads. If the area under the water meniscus as shown by the shaded area in Figure A.1 is rotated around the point of contact between the two spherical glass beads, we will get the volume of a single water bridge.

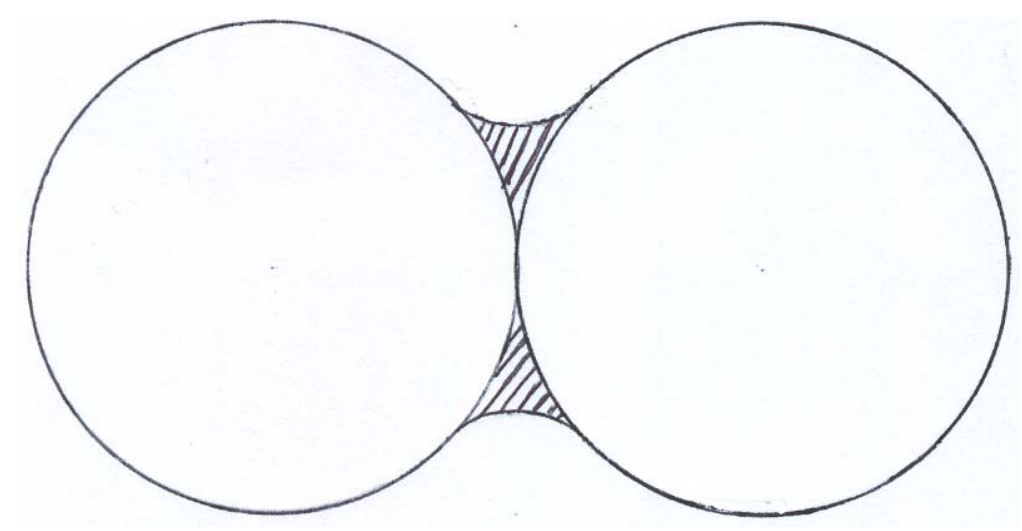

Figure A.1. Model of a single water bridge.

The calculus of the area of surface of revolution and the volume of solid of revolution is as follows. Let us suppose we have the function $y=f(x)$ as shown in Figure A.2. The length of the curve between $x$ and $(x+d x)$ is $d L$, and $d L$ can be calculated using Equation (A.1). 


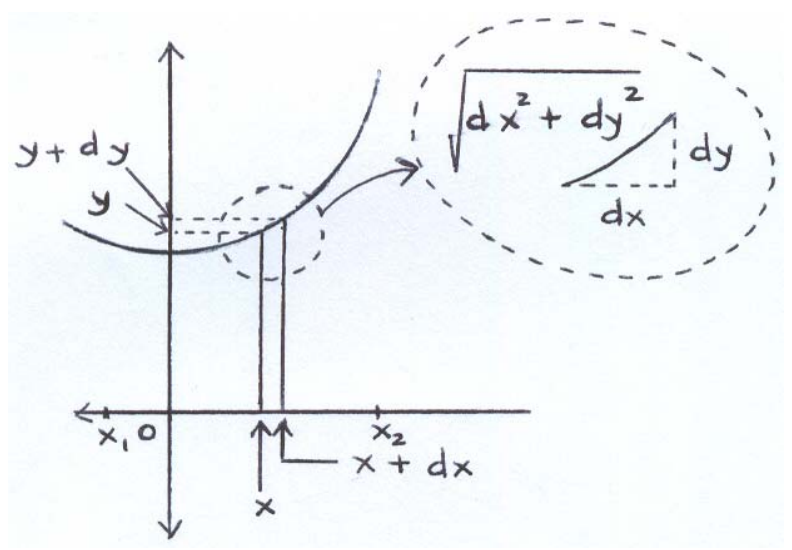

Figure A.2. Curve of $y=f(x)$.

$$
d L=\sqrt{d x^{2}+d y^{2}}
$$

The area of the surface of revolution of the curve with length $d L$ around the $x$-axis is equal to $d A_{\text {single }}$, and $d A_{\text {single }}$ is calculated using Equation (A.2).

$$
d A_{\text {sin } g l e}=2 \pi y(d L)=2 \pi y \sqrt{d x^{2}+d y^{2}}=2 \pi y\left\{\sqrt{1+\left(\frac{d y}{d x}\right)^{2}}\right\} d x
$$

Then, the area of the surface of revolution of the curve from $\left(x_{1}, y_{1}\right)$ to $\left(x_{2}, y_{2}\right)$ around the $x$-axis is $A_{\text {single }}$ that is calculated as follows.

$$
A_{\text {sin } g l e}=\int_{x_{1}}^{x_{2}}\left\{2 \pi y \sqrt{1+\left(\frac{d y}{d x}\right)^{2}}\right\} d x
$$

Similarly, we can derive the equation of the volume of solid of revolution as follows. The volume of a thin slice of the solid of revolution obtained by rotating the part of the curve from $(x, y)$ to $(x+d x, y+d y)$ around the $x$-axis is $d V_{\text {revolution }}$ that can be calculated using Equation (A.4).

$$
d V_{\text {revolution }}=\pi y^{2} d x
$$


Then, volume of the solid of revolution obtained by rotating the curve from $\left(x_{l}, y_{l}\right)$ to $\left(x_{2}, y_{2}\right)$ around the $x$-axis is $V_{\text {revolution, }}$ that is Equation (A.5).

$$
V_{\text {revolution }}=\int_{x_{1}}^{x_{2}} \pi y^{2} d x
$$

Equations (A.3) and (A.5) can be used to derive the equations of the surface area and the volume of a single water bridge.

\section{A.1. Surface area of a single water bridge}

The geometry of the model of a single water bridge is shown in Figure A.3. We need to derive the relation between $y$ and $x$ first, before we can integrate Equations (A.3) and (A.5). The detail of Figure A.3 is shown more clearly in Figure A.4.

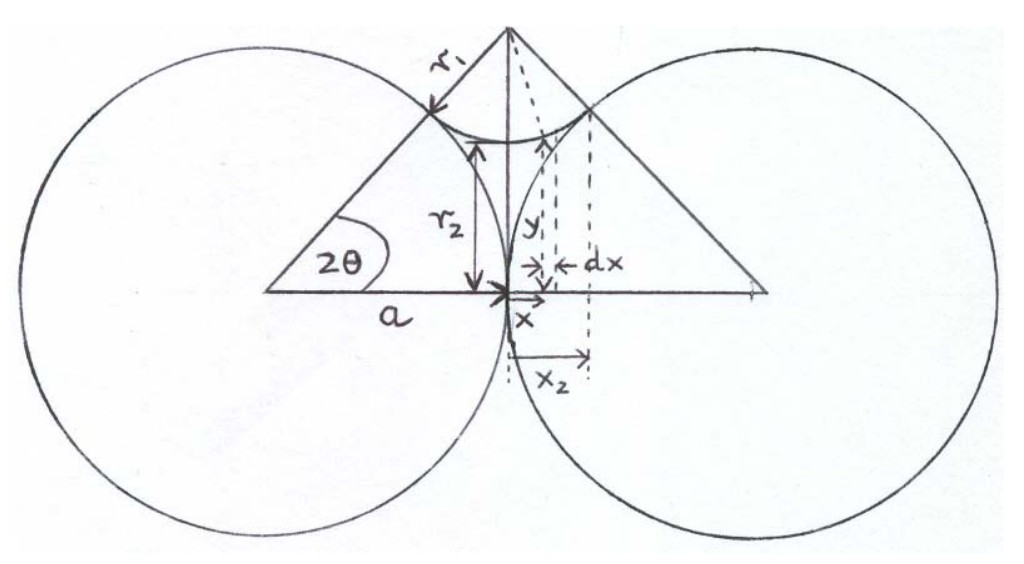

Figure A.3. Geometry of the model of a single water bridge.

Based on Figure A.4, we have the following relations.

$$
\cos 2 \theta=\frac{a}{a+r_{1}}
$$



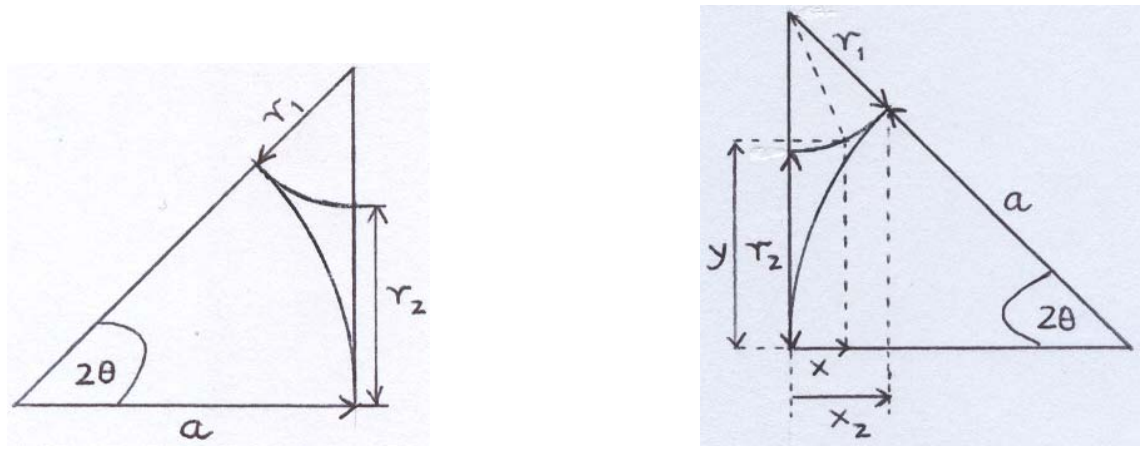

Figure A.4. Detail of the geometry of the model of a single water bridge.

$$
\tan 2 \theta=\frac{r_{1}+r_{2}}{a}
$$

Equation (A.6) can be rearranged to get the radius of the meniscus of a water bridge $\left(r_{1}\right)$.

$$
r_{1}=a \frac{(1-\cos 2 \theta)}{\cos 2 \theta}
$$

If we substitute Equation (A.8) into Equation (A.7) and rearrange the equation, we will get the equation of the shortest distance between the point of contact of the two glass bead particles and the meniscus of the water bridge $\left(r_{2}\right)$.

$$
r_{2}=a \frac{(\sin 2 \theta+\cos 2 \theta-1)}{\cos 2 \theta}
$$

Then the relation between $y$ and $x$ can be expressed as Equation (A.10).

$$
y=r_{1}+r_{2}-\sqrt{r_{1}^{2}-x^{2}}
$$

From Equation (A.10), we get Equation (A.11).

$$
\frac{d y}{d x}=\frac{x}{\sqrt{r_{1}^{2}-x^{2}}}
$$

Then using Equation (A.11), we get Equation (A.12). 


$$
\sqrt{1+\left(\frac{d y}{d x}\right)^{2}}=\frac{r_{1}}{\sqrt{r_{1}^{2}-x^{2}}}
$$

We can combine Equations (A.3), (A.10), and (A.12) to get the surface area of a single water bridge $\left(A_{\text {single }}\right)$ with $x_{1}=-x_{2}$.

$$
A_{\text {sin gle }}=\int_{x_{2}}^{x_{2}} 2 \pi\left(r_{1}+r_{2}-\sqrt{r_{1}^{2}-x^{2}}\right) \frac{r_{1}}{\sqrt{r_{1}^{2}-x^{2}}} d x=4 \pi r_{1} \int_{0}^{x_{2}}\left\{\frac{r_{1}+r_{2}}{\sqrt{r_{1}^{2}-x^{2}}}-1\right\} d x
$$

The projection of the meniscus of a single water bridge on the line that connects the center points of the two glass beads gives a line of length $2 x_{2}$, with $x_{2}$ can be calculated using Equation (A.14).

$$
x_{2}=a(1-\cos 2 \theta)
$$

To solve Equation (A.13), we do the following mathematics.

1. Using Equations (A.8) and (A.9), find: $\left(r_{1}+r_{2}\right)$

$$
r_{1}+r_{2}=a \frac{(1-\cos 2 \theta)}{\cos 2 \theta}+a \frac{(\sin 2 \theta+\cos 2 \theta-1)}{\cos 2 \theta}=\operatorname{atan} 2 \theta
$$

2. Using Equations (A.8) and (A.14), find:

$$
\int_{0}^{x_{2}} \frac{1}{\sqrt{r_{1}^{2}-x^{2}}} d x
$$

Let,

$$
\begin{aligned}
x=r_{1} \sin w & \rightarrow x=0, w=0 ; x=x_{2}, w=w_{2} \\
& \rightarrow \sin w_{2}=\frac{x_{2}}{r_{1}}=\frac{a(1-\cos 2 \theta)}{a(1-\cos 2 \theta)} \cos 2 \theta=\cos 2 \theta \rightarrow w_{2}=\left(\frac{\pi}{2}-2 \theta\right) \\
& \rightarrow d x=r_{1} \cos w d w
\end{aligned}
$$




$$
\begin{gathered}
\rightarrow \sqrt{r_{1}^{2}-x^{2}}=\sqrt{r_{1}^{2}\left(1-\sin ^{2} w\right)}=r_{1} \cos w \\
\int_{0}^{x_{2}} \frac{1}{\sqrt{r_{1}^{2}-x^{2}}} d x=\int_{0}^{v_{2}} \frac{r_{1} \cos w}{r_{1} \cos w} d w=\int_{0}^{w_{2}} d w=w_{2}=\left(\frac{\pi}{2}-2 \theta\right)
\end{gathered}
$$

3. Using Equation (A.14), find:

$$
\int_{0}^{x_{2}} d x=x_{2}=a(1-\cos 2 \theta)
$$

4. Substitute Equations (A.8), (A.15) to (A.17) into Equation (A.13).

$$
A_{\text {sin } g l e}=4 \pi r_{1} \int_{0}^{x_{2}}\left\{\frac{r_{1}+r_{2}}{\sqrt{r_{1}^{2}-x^{2}}}-1\right\} d x=4 \pi a \frac{(1-\cos 2 \theta)}{\cos 2 \theta}\left\{\operatorname{atan} 2 \theta\left(\frac{\pi}{2}-2 \theta\right)-a(1-\cos 2 \theta)\right\}
$$

We have the following identity,

$$
\cos 2 \theta=1-2 \sin ^{2} \theta=2 \cos ^{2} \theta-1
$$

By substitution of Equation (A.19) into Equation (A.18), we obtain the final expression for $A_{\text {single }}$.

$$
A_{\text {sin } g l e}=\frac{8 \pi a^{2} \sin ^{2} \theta}{\cos 2 \theta}\left\{\tan 2 \theta\left(\frac{\pi}{2}-2 \theta\right)-2 \sin ^{2} \theta\right\}
$$

\section{A.2. Volume of a single water bridge}

Let $V_{l}$ be the volume of the solid of revolution obtained by rotating the water meniscus around the axis that connects the center points of the two spherical glass beads. Using Equations (A.5) and (A.10), we can write the equation of $V_{l}$ as follows. 


$$
\begin{aligned}
& V_{1}=\int_{-x_{2}}^{x_{2}} \pi y^{2} d x=2 \pi \int_{0}^{x_{2}}\left\{r_{1}+r_{2}-{\sqrt{r_{1}^{2}-x^{2}}}^{2} d x\right. \\
& V_{1}=2 \pi \int_{0}^{x_{2}}\left\{\left(r_{1}+r_{2}\right)^{2}+r_{1}^{2}-2\left(r_{1}+r_{2}\right) \sqrt{r_{1}^{2}-x^{2}}-x^{2}\right\} d x
\end{aligned}
$$

To solve Equation (A.21), we do the following mathematics.

1. Using Equations (A.8) and (A.15), find: $\left(r_{1}+r_{2}\right)^{2}+r_{l}{ }^{2}$

$$
\begin{aligned}
\left(r_{1}+r_{2}\right)^{2}+r_{1}^{2} & =a^{2} \tan ^{2} 2 \theta+a^{2} \frac{(1-\cos 2 \theta)^{2}}{\cos ^{2} 2 \theta} \\
& =\frac{a^{2}}{\cos ^{2} 2 \theta}\left(\sin ^{2} 2 \theta+1-2 \cos 2 \theta+\cos ^{2} 2 \theta\right) \\
& =\frac{2 a^{2}}{\cos ^{2} 2 \theta}(1-\cos 2 \theta)
\end{aligned}
$$

2. Using Equations (A.14) and (A.22), find:

$$
\int_{0}^{x_{2}}\left\{\left(r_{1}+r_{2}\right)^{2}+r_{1}^{2}\right\} d x=\frac{2 a^{2}}{\cos ^{2} 2 \theta}(1-\cos 2 \theta) x_{2}=\frac{2 a^{3}}{\cos ^{2} 2 \theta}(1-\cos 2 \theta)^{2}
$$

3. Using Equations (A.8), (A.14), and (A.19), find:

$$
\int_{0}^{x_{2}} \sqrt{r_{1}^{2}-x^{2}} d x
$$

Let,

$$
\begin{aligned}
x=r_{1} \sin w & \rightarrow x=0, w=0 ; x=x_{2}, w=w_{2} \\
& \rightarrow \sin w_{2}=\frac{x_{2}}{r_{1}}=\frac{a(1-\cos 2 \theta)}{a(1-\cos 2 \theta)} \cos 2 \theta=\cos 2 \theta \rightarrow w_{2}=\left(\frac{\pi}{2}-2 \theta\right) \\
& \rightarrow d x=r_{1} \cos w d w
\end{aligned}
$$




$$
\begin{aligned}
& \rightarrow \sqrt{r_{1}^{2}-x^{2}}=\sqrt{r_{1}^{2}\left(1-\sin ^{2} w\right)}=r_{1} \cos w \\
& \int_{0}^{r_{2}} \sqrt{r_{1}^{2}-x^{2}} d x=\int_{0}^{v_{2}} r_{1} \cos w r_{1} \cos w d w=\int_{0}^{v_{2}} r_{1}^{2} \cos ^{2} w d w=\frac{r_{1}^{2}}{2} \int_{0}^{v_{2}}(1+\cos 2 w) d w \\
&=\frac{r_{1}^{2}}{2}\left(w_{2}+\frac{1}{2} \sin 2 w_{2}\right)=\frac{a^{2}}{2} \frac{(1-\cos 2 \theta)^{2}}{\cos ^{2} 2 \theta}\left\{\left(\frac{\pi}{2}-2 \theta\right)+\frac{1}{2} \sin (\pi-4 \theta)\right\} \\
&=\frac{a^{2}}{2} \frac{\left(1-\cos ^{2} 2 \theta\right)^{2}}{\cos ^{2} 2 \theta}\left\{\left(\frac{\pi}{2}-2 \theta\right)+\frac{1}{2} \sin 4 \theta\right\}
\end{aligned}
$$

4. Using Equations (A.15), (A.24), and the following identity, $\sin 4 \theta=2 \sin 2 \theta \cos 2 \theta$

find:

$$
\begin{aligned}
\int_{0}^{x_{2}} 2\left(r_{1}+r_{2}\right) \sqrt{r_{1}^{2}-x^{2}} d x & =2(\operatorname{atan} 2 \theta) \frac{a^{2}}{2} \frac{(1-\cos 2 \theta)^{2}}{\cos ^{2} 2 \theta}\left\{\left(\frac{\pi}{2}-2 \theta\right)+\frac{1}{2} \sin 4 \theta\right\} \\
& =a^{3} \frac{(1-\cos 2 \theta)^{2}}{\cos ^{2} 2 \theta}\left\{\tan 2 \theta\left(\frac{\pi}{2}-2 \theta\right)+\tan 2 \theta(\sin 2 \theta \cos 2 \theta)\right\} \\
& =a^{3} \frac{(1-\cos 2 \theta)^{2}}{\cos ^{2} 2 \theta}\left\{\tan 2 \theta\left(\frac{\pi}{2}-2 \theta\right)+\sin ^{2} 2 \theta\right\}
\end{aligned}
$$

5. Using Equation (A.14), find:

$$
\int_{0}^{x_{2}} x^{2} d x=\frac{1}{3} x_{2}{ }^{3}=\frac{a^{3}}{3}(1-\cos 2 \theta)^{3}
$$

6. Substitute Equations (A.23), (A.26), and (A.27) into Equation (A.21).

$$
\frac{V_{1}}{2 \pi}=\int_{0}^{x_{2}}\left\{\left(r_{1}+r_{2}\right)^{2}+r_{1}^{2}-2\left(r_{1}+r_{2}\right) \sqrt{r_{1}^{2}-x^{2}}-x^{2}\right\} d x
$$




$$
=\frac{2 a^{3}}{\cos ^{2} 2 \theta}(1-\cos 2 \theta)^{2}-a^{3} \frac{(1-\cos 2 \theta)^{2}}{\cos ^{2} 2 \theta}\left\{\tan 2 \theta\left(\frac{\pi}{2}-2 \theta\right)+\sin ^{2} 2 \theta\right\}-\frac{a^{3}}{3}(1-\cos 2 \theta)^{3}
$$

Now, let $V_{2}$ be the volume of the solid of revolution obtained by rotating the wetted glass bead surface around the axis that connects the center points of the two spherical glass beads. The geometry of a half wetted surface of a glass bead particle is shown in Figure A.5. Based on the figure, we can derive the relation between $y$ and $x$ to get Equation (A.29).

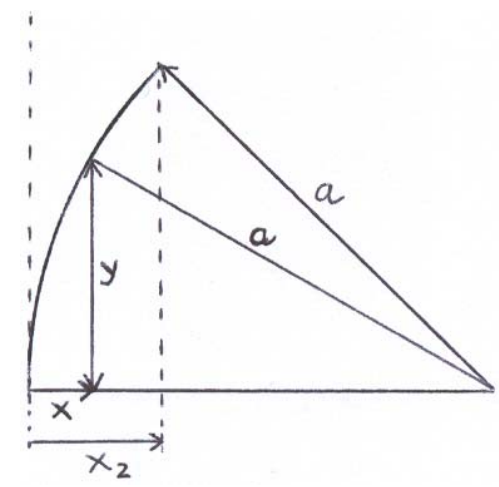

Figure A.5. Geometry of a half wetted surface of a glass bead particle.

$$
y=\sqrt{a^{2}-(a-x)^{2}}=\sqrt{a^{2}-a^{2}+2 a x-x^{2}}=\sqrt{2 a x-x^{2}}
$$

Using Equations (A.5), (A.14), and (A.29), we can write the equation of $V_{2}$ as follows.

$$
\begin{aligned}
& V_{2}=\int_{-x_{2}}^{x_{2}} \pi y^{2} d x=2 \pi \int_{0}^{x_{2}}\left(2 a x-x^{2}\right) d x=2 \pi\left(a x_{2}^{2}-\frac{1}{3} x_{2}^{3}\right) \\
& \frac{V_{2}}{2 \pi}=a^{3}(1-\cos 2 \theta)^{2}-\frac{a^{3}}{3}(1-\cos 2 \theta)^{3}
\end{aligned}
$$


Finally, the volume of a single water bridge $\left(V_{\text {single }}\right)$ can be calculated using Equations (A.28) and (A.30) as follows.

$$
\begin{aligned}
& \frac{V_{\text {sin } g l e}}{2 \pi}=\frac{V_{1}}{2 \pi}-\frac{V_{2}}{2 \pi} \\
= & \frac{2 a^{3}}{\cos ^{2} 2 \theta}(1-\cos 2 \theta)^{2}-a^{3} \frac{(1-\cos 2 \theta)^{2}}{\cos ^{2} 2 \theta}\left\{\tan 2 \theta\left(\frac{\pi}{2}-2 \theta\right)+\sin ^{2} 2 \theta\right\}-\frac{a^{3}}{3}(1-\cos 2 \theta)^{3} \\
& -a^{3}(1-\cos 2 \theta)^{2}+\frac{a^{3}}{3}(1-\cos 2 \theta)^{3} \\
= & \frac{2 a^{3}}{\cos ^{2} 2 \theta}(1-\cos 2 \theta)^{2}-a^{3} \frac{(1-\cos 2 \theta)^{2}}{\cos ^{2} 2 \theta}\left\{\tan 2 \theta\left(\frac{\pi}{2}-2 \theta\right)+\sin ^{2} 2 \theta\right\}-a^{3}(1-\cos 2 \theta)^{2} \\
= & \frac{a^{3}(1-\cos 2 \theta)^{2}}{\cos ^{2} 2 \theta}\left\{2-\tan 2 \theta\left(\frac{\pi}{2}-2 \theta\right)-\sin ^{2} 2 \theta-\cos ^{2} 2 \theta\right\}
\end{aligned}
$$

We know that

$$
\sin ^{2} 2 \theta+\cos ^{2} 2 \theta=1
$$

By substitution of Equations (A.19) and (A.32) into Equation (A.31), we get the final expression of $V_{\text {single }}$ as follows.

$$
\begin{aligned}
& \frac{V_{\text {sin gle }}}{2 \pi}=\frac{a^{3}\left(2 \sin ^{2} \theta\right)^{2}}{\cos ^{2} 2 \theta}\left\{1-\tan 2 \theta\left(\frac{\pi}{2}-2 \theta\right)\right\} \\
& V_{\text {sin gle }}=\frac{8 \pi a^{3} \sin ^{4} \theta}{\cos ^{2} 2 \theta}\left\{1-\tan 2 \theta\left(\frac{\pi}{2}-2 \theta\right)\right\}
\end{aligned}
$$




\section{APPENDIX B}

\section{ENGLEZOS ET AL.'S MODEL AND ITS MODIFICATION}

Englezos et al. (1987a) did their experiments of MGH and EGH formations in a semi batch stirred tank reactor that was operated at isothermal and isobaric conditions. The experiments were done at four temperatures, i.e. $274,276,279$, and $282 \mathrm{~K}$, and pressure range from 3.29 to $8.90 \mathrm{MPa}$ for $\mathrm{MGH}$ and 0.64 to $2.19 \mathrm{MPa}$ for EGH. Their kinetic model was based on the crystallization theory, with the interfacial mass transfer model was based on the film theory.

\section{B.1. Total growth rate of NGH crystals}

To model the growth of NGH crystals, Englezos et al. considered the process consists of two consecutive steps as shown in Figure B.1, with no accumulation in the laminar diffusion layer around the spherical NGH crystal.

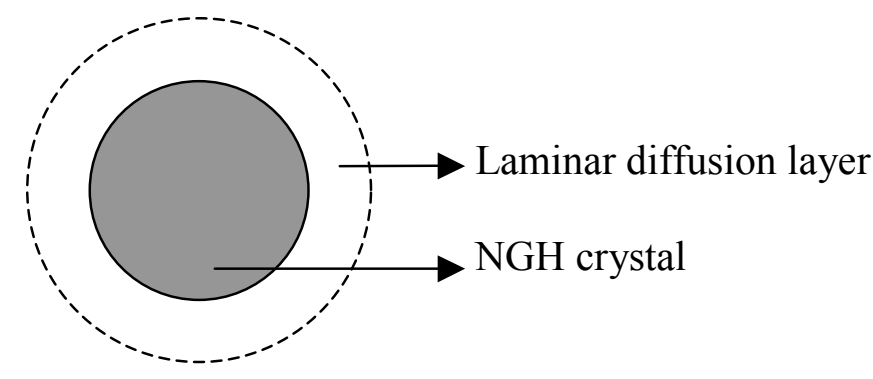

Figure B.1. Model of NGH crystal growth. 
Step 1: Diffusion of the dissolved NG from the bulk of the solution to the NGH crystalliquid interface through the laminar diffusion layer around a NGH crystal. This step can be expressed as Equation (B.1).

$$
\left(\frac{d n}{d t}\right)_{p}=k_{d} A_{p}\left(f-f_{i}\right)
$$

Step 2: Incorporation of the dissolved NG molecules into the water molecules and the subsequent stabilization of the framework of the structured water at the NGH crystalliquid interface. This step is shown as Equation (B.2).

$$
\left(\frac{d n}{d t}\right)_{p}=k_{r} A_{p}\left(f_{i}-f_{e q}\right)
$$

If we combine Equations (B.1) and (B.2), we will get the equation of the growth rate of a NGH crystal as follows.

$$
\left(\frac{d n}{d t}\right)_{p}=K^{*} A_{p}\left(f-f_{e q}\right)
$$

where

$$
\frac{1}{K^{*}}=\frac{1}{k_{r}}+\frac{1}{k_{d}}
$$

The equation contains an assumption that the outside surface of the surrounding layer is equal to the surface of the NGH crystal.

The total reaction rate $R_{y}(t)$ is obtained by integration of the rate per NGH crystal over all NGH crystals of any sizes.

$$
R_{y}(t)=\int_{0}^{\infty}\left(\frac{d n}{d t}\right)_{p} \phi(r, t) d r=\int_{0}^{\infty} K^{*} A_{p}\left(f-f_{e q}\right) \phi(r, t) d r
$$




$$
=\int_{0}^{\infty} K * 4 \pi r^{2}\left(f-f_{e q}\right) \phi(r, t) d r=4 \pi K * \mu_{2}\left(f-f_{e q}\right)
$$

where $\mu_{2}$ is the second moment of the crystal size distribution $\phi(r, t)$, and is given by

$$
\mu_{2}=\int_{0}^{\infty} r^{2} \phi(r, t) d r
$$

\section{B.2. Rate of NG consumption from the gas phase}

The rate of $\mathrm{NG}$ consumption from the gas phase is equal to the rate of transportation of the NG from the gas phase to the liquid phase. Film theory is used to model the interfacial mass transfer. Quasi-steady-state mass balance of the dissolved NG in the liquid film of thickness $y_{L}$ at gas-liquid interface will give the following equation.

$$
D_{L} \frac{d^{2} c}{d y^{2}}=4 \pi K * \mu_{2}\left(f-f_{e q}\right)
$$

The relation between concentration of the dissolved NG and its fugacity in the gas phase can be expressed by Equation (B.8).

$$
c=\frac{c_{w 0} f}{H^{*}}
$$

If concentration of water liquid is assumed to be constant and is equal to its initial concentration $\left(c_{w 0}\right)$, then Equation (B.7) can be written as Equation (B.9).

$$
D * \frac{d^{2} Y}{d y^{2}}=4 \pi K * \mu_{2} Y
$$

where

$$
Y=f-f_{e q}, \quad D^{*}=\frac{D_{L} c_{w 0}}{H^{*}}
$$


Equation (B.9) satisfies the following boundary conditions.

$$
\begin{array}{lll}
y=0 & \rightarrow & Y=f_{g}-f_{e q} \\
y=y_{L} & \rightarrow & Y=f_{b}-f_{e q}
\end{array}
$$

It is assumed here that the gas-phase resistance is negligible. The analytical solution of Equation (B.9) is Equation (B.13).

$$
Y=C_{1} \sinh \left(\gamma \frac{y}{y_{L}}\right)+C_{2} \cosh \left(\gamma \frac{y}{y_{L}}\right)
$$

with

$$
\gamma=y_{L} \sqrt{\frac{4 \pi K^{*} \mu_{2}}{D^{*}}}
$$

Using the boundary conditions (B.11) and (B.12), we obtain the integration constants $C_{1}$ and $C_{2}$.

$$
\begin{aligned}
& C_{1}=\frac{\left(f_{b}-f_{e q}\right)-\left(f_{g}-f_{e q}\right) \cosh \gamma}{\sinh \gamma} \\
& C_{2}=f_{g}-f_{e q}
\end{aligned}
$$

Combining Equations (B.13), (B.15), and (B.16) gives Equation (B.17).

$$
\begin{aligned}
Y & =\frac{\left(f_{b}-f_{e q}\right)}{\sinh \gamma} \sinh \left(\gamma \frac{y}{y_{L}}\right)+\frac{\left(f_{g}-f_{e q}\right)}{\sinh \gamma}\left\{\sinh \gamma \cosh \left(\gamma \frac{y}{y_{L}}\right)-\cosh \gamma \sinh \left(\gamma \frac{y}{y_{L}}\right)\right\} \\
& =\frac{\left(f_{b}-f_{e q}\right)}{\sinh \gamma} \sinh \left(\gamma \frac{y}{y_{L}}\right)+\frac{\left(f_{g}-f_{e q}\right)}{\sinh \gamma} \sinh \left\{\gamma\left(1-\frac{y}{y_{L}}\right)\right\}
\end{aligned}
$$

Using the definition of $Y$ as shown in Equation (B.10), we finally get Equation (B.18).

$$
f=f_{e q}+\left\{\left(f_{g}-f_{e q}\right) \sinh \left[\gamma\left(1-\frac{y}{y_{L}}\right)\right]+\left(f_{b}-f_{e q}\right) \sinh \left(\gamma \frac{y}{y_{L}}\right)\right\} \frac{1}{\sinh \gamma}
$$


Furthermore, we can derive the equation of the flux at gas-liquid interface.

$$
\begin{aligned}
& (J)_{y=0}=-D_{L}\left(\frac{d c}{d y}\right)_{y=0}=-D *\left(\frac{d f}{d y}\right)_{y=0} \\
& (J)_{y=0}=\left(\frac{D * \gamma}{y_{L}}\right) \frac{\left\lfloor\left(f_{g}-f_{e q}\right) \cosh \gamma-\left(f_{b}-f_{e q}\right)\right\rfloor}{\sinh \gamma}
\end{aligned}
$$

Finally, the rate of NG consumption from the gas phase is equal to the mass transfer rate of the NG through the gas-liquid interface, as shown in the following equation.

$$
\frac{d n}{d t}=(J)_{y=0} A(g-l)=\left(\frac{D^{*} \gamma A(g-l)}{y_{L}}\right) \frac{\left\lfloor\left(f_{g}-f_{e q}\right) \cosh \gamma-\left(f_{b}-f_{e q}\right)\right\rfloor}{\sinh \gamma}
$$

\section{B.3. Mass balance of the dissolved NG in the bulk of the liquid}

The total input of dissolved NG to the bulk of the liquid is equal to the transportation of the dissolved NG across the area of mass transfer at $y=y_{L}$. The flux at $y=y_{L}$ can be expressed as Equation (B.21).

$$
(J)_{y=y_{L}}=-D^{*}\left(\frac{d f}{d y}\right)_{y=y_{L}}=\left(\frac{D * \gamma}{y_{L}}\right) \frac{\left\lfloor\left(f_{g}-f_{e q}\right)-\left(f_{b}-f_{e q}\right) \cosh \gamma\right\rfloor}{\sinh \gamma}
$$

The mass transfer rate of dissolved NG across mass transfer area at $y=y_{L}$ is equal to the total rate of accumulation of the dissolved NG in the bulk of liquid and the rate of dissolved NG consumption into NGH.

$$
(J)_{y=y_{L}} a^{*}=\frac{d}{d t}\left(\frac{c_{w 0} f_{b}}{H^{*}}\right)+4 \pi K * \mu_{2}\left(f_{b}-f_{e q}\right)
$$

Here, $a *$ is the gas-liquid interfacial area per unit of liquid volume. Combining Equations (B.21) and (B.22) gives Equation (B.23). 


$$
\frac{d f_{b}}{d t}=\frac{H^{*} D^{*} \gamma a^{*}}{c_{w 0} y_{L} \sinh \gamma}\left\{\left(f_{g}-f_{e q}\right)-\left(f_{b}-f_{e q}\right) \cosh \gamma\right\}-\frac{4 \pi K^{*} \mu_{2} H^{*}}{c_{w 0}}\left(f_{b}-f_{e q}\right)
$$

\section{B.4. Estimation of $\mu_{2}(t)$}

$\mu_{2}$ is the second moment of the NGH crystal size distribution, and is given by Equation (B.6). To estimate $\mu_{2}(t)$, a population balance proposed by Kane et al. (1974) is used as shown by Equation (B.24).

$$
\frac{\partial(\phi(r, t) d r)}{\partial t}=\left[\left.G \phi(r, t)\right|_{r}-\left.G \phi(r, t)\right|_{r+d r}\right]+\beta \psi(r, t) d r
$$

where $\phi(r, t)$ is crystal size distribution, $\phi(r, t) d r$ is the number of crystals per unit volume in the size range $r$ to $r+d r, G$ is the linear growth rate of the crystals $(\partial r / \partial t), \beta$ is the nucleation rate per unit volume, and $\psi(r, t) d r$ is the fraction of the crystals born in the size range from $r$ to $r+d r$. Hence, Equation (B.24) tells us that the rate of change in the number of crystals in the size range $r$ to $r+d r$ is equal to the rate of number of crystals growing into the size range minus the rate of number of crystals growing out of the size range, plus the rate of number of newly-born nuclei nucleated into the size range. The equation can be rearranged as Equation (B.25).

$$
\frac{\partial \phi(r, t)}{\partial t}+\frac{\partial(G \phi(r, t))}{\partial r}=\beta \psi(r, t)
$$

Then, it is assumed that,

1. the linear growth rate is independent of the crystal size;

2. new crystals are nucleated at near zero size; 
3. nucleation rate is proportional to the second moment of the crystal size distribution, as shown in Equation (B.26) with $\alpha_{2}$ is the nucleation rate constant.

$$
\beta=\alpha_{2} \int_{0}^{\infty} r^{2} \phi(r, t) d r=\alpha_{2} \mu_{2}
$$

Applying these assumptions on Equation (B.25) gives Equation (B.27).

$$
\frac{\partial \phi(r, t)}{\partial t}+G \frac{\partial \phi(r, t)}{\partial r}=\alpha_{2} \mu_{2} \delta(r-0)
$$

where $\delta(r-0)$ is the Dirac delta function. Multiplying Equation (B.27) by $r^{m}$ and integrating with respect to $r$ between the limits 0 to $\infty$ give the following equation.

$$
\begin{aligned}
& \frac{\partial}{\partial t} \int_{0}^{\infty} r^{m} \phi(r, t) d r+G \int_{0}^{\infty} r^{m} \frac{\partial \phi(r, t)}{\partial r} d r=\alpha_{2} \mu_{2} \int_{0}^{\infty} r^{m} \delta(r-0) d r \\
& \frac{d}{d t} \mu_{m}+G\left\{\left.r^{m} \phi(r, t)\right|_{0} ^{\infty}-\int_{0}^{\infty} m r^{m-1} \phi(r, t) d r\right\}=\alpha_{2} \mu_{2} \delta_{o m} \\
& \frac{d \mu_{m}}{d t}=m G \mu_{m-1}+\alpha_{2} \mu_{2} \delta_{o m}
\end{aligned}
$$

where $\delta_{\text {om }}$ is the Kronecker delta. Equation (B.28) can be rewritten as a set of three ordinary differential equations as follows.

$$
\begin{aligned}
& \frac{d \mu_{0}}{d t}=\alpha_{2} \mu_{2} \\
& \frac{d \mu_{1}}{d t}=G \mu_{0} \\
& \frac{d \mu_{2}}{d t}=2 G \mu_{1}
\end{aligned}
$$




\section{B.5. The average of the linear growth rate}

The linear growth rate $(G)$ is a function of time and distance from the gas-liquid interface, but not a function of the size of the crystals. Englezos et al. define the average linear growth rate $\left(G_{a v g}\right)$ as follows.

$$
G_{a v g}=\left(\frac{1}{L}\right)\left[\int_{0}^{y_{L}} G d y+G_{b}\left(L-y_{L}\right)\right]=\left(\frac{1}{L}\right)\left[\int_{0}^{y_{L}}\left(\frac{d r}{d t}\right) d y+\left(\frac{d r}{d t}\right)_{b}\left(L-y_{L}\right)\right]
$$

where $L$ is the distance between the gas-liquid interface and the bottom of the reactor. Using Equation (B.3), we can derive the equation of the linear growth rate $(G)$ as follows. Let $M_{H}$ be the molecular weight of the $\mathrm{NGH}$ of the form $\mathrm{X} \cdot n_{w} \mathrm{H}_{2} \mathrm{O}$, and $\rho_{H}$ is the $\mathrm{NGH}$ density. The change in the volume of NGH can be written as follows.

$$
\begin{aligned}
& \frac{M_{H}}{\rho_{H}}\left(\frac{d n}{d t}\right)_{p}=\left(\frac{d V}{d t}\right)_{p}=\frac{d}{d t}\left(\frac{4}{3} \pi r^{3}\right)_{p}=\frac{M_{H}}{\rho_{H}} K * 4 \pi r^{2}\left(f-f_{e q}\right) \\
& G=\frac{d r}{d t}=\frac{K^{*} M_{H}\left(f-f_{e q}\right)}{\rho_{H}}
\end{aligned}
$$

Substitution of Equation (B.33) into Equation (B.32) gives Equation (B.34).

$$
G_{a v g}=\left(\frac{K^{*} M_{H}}{\rho_{H} L}\right)\left[\int_{0}^{y_{L}}\left(f-f_{e q}\right) d y+\left(f_{b}-f_{e q}\right)\left(L-y_{L}\right)\right]
$$

Using Equation (B.18), we can do the following integration.

$$
\begin{aligned}
\int_{0}^{y_{L}}\left(f-f_{e q}\right) d y & =\frac{1}{\sinh \gamma} \int_{0}^{y_{L}}\left\{\left(f_{g}-f_{e q}\right) \sinh \left[\gamma\left(1-\frac{y}{y_{L}}\right)\right]+\left(f_{b}-f_{e q}\right) \sinh \left(\gamma \frac{y}{y_{L}}\right)\right\} d y \\
& =\frac{y_{L}}{\gamma \sinh \gamma}\left(f_{g}+f_{b}-2 f_{e q}\right)(\cosh \gamma-1)
\end{aligned}
$$


Combining Equations (B.34) and (B.35) gives Equation (B.36).

$$
G_{a v g}=\left(\frac{K^{*} M_{H}}{\rho_{H} L}\right)\left(y_{L} \frac{\left(f_{g}+f_{b}-2 f_{e q}\right)(\cosh \gamma-1)}{\gamma \sinh \gamma}+\left(L-y_{L}\right)\left(f_{b}-f_{e q}\right)\right)
$$

\section{B.6. Determination of model parameters}

The Englezos et al.'s model represents the growth period of NGH formation. To solve the five differential equations, i.e. Equations (B.20), (B.23), (B.29) to (B.31), some parameters, including initial conditions, must be measured or calculated. The initial conditions for Equations (B.20) and (B.23) are as follows.

$$
\begin{aligned}
& n(t=0)=n_{t b} \\
& f_{b}(t=0)=f_{e q}
\end{aligned}
$$

where $n_{t b}$ is the measured number of moles of NG that have been dissolved at the turbidity point, and $f_{e q}$ is the minimum fugacity for $\mathrm{NGH}$ to exist that is equal to the equilibrium fugacity. The turbidity point is the time when turbidity starts to appear in the liquid phase. The turbidity in the liquid phase is caused by sudden appearance of NGH particles. The time when turbidity starts to appear is assigned as $t=0$. The equilibrium fugacity is calculated using an appropriate thermodynamic model for NGH equilibrium at temperature of the experiment.

The initial condition for Equation (B.29) $\left(\mu_{0}^{0}\right)$ is the number of NGH particles per unit volume of liquid phase at $t=0$. If $n_{e q}$ is the amount of NG that would have been dissolved at the equilibrium pressure of NGH formation and temperature of experiment, then $\left(n_{t b}-n_{e q}\right)$ is the amount of dissolved NG that is converted to NGH at $t=0$. Let $N_{p}$ be 
the number of NGH particles, and $r_{c r}$ be the radius of NGH particles. Then, we can derive the equation of $N_{p}$ as follows.

$$
\begin{aligned}
& N_{p}\left(\frac{4}{3} \pi r_{c r}^{3}\right) \frac{\rho_{H}}{M_{H}}=n_{t b}-n_{e q} \\
& N_{p}=\frac{3 M_{H}\left(n_{t b}-n_{e q}\right)}{4 \pi \rho_{H} r_{c r}{ }^{3}}
\end{aligned}
$$

This equation contains an assumption that all NGH particles are spherical with identical radius. Then, $\mu_{0}^{0}$ can be calculated using Equation (B.40).

$$
\mu_{0}^{0}=\frac{3 M_{H}\left(n_{t b}-n_{e q}\right)}{4 \pi V_{L} \rho_{H} r_{c r}{ }^{3}}
$$

The initial conditions for Equations (B.30) and (B.31) according Englezos et al. (1987a) are Equations (B.41) and (B.42).

$$
\begin{aligned}
& \mu_{1}^{0}=2 r_{c r} \mu_{0}^{0} \\
& \mu_{2}^{0}=4 r_{c r}^{2} \mu_{0}^{0}
\end{aligned}
$$

Knowing the liquid volume $\left(V_{L}\right)$, Henry's constant $\left(H^{*}\right)$, the equilibrium fugacity $\left(f_{e q}\right)$, and the initial concentration of water $\left(c_{w 0}\right)$, we can calculate $n_{e q}$ using Equation (B.8), with

$$
n_{e q}=c_{e q} V_{L}
$$

The radius of NGH particles at turbidity point $\left(r_{c r}\right)$ is equal to the critical radius of NGH particles, which is the minimum radius of a nucleus for continuous crystal growth. The critical radius is calculated based on nucleation theory as shown in Equation (B.44).

$$
r_{c r}=-\frac{2 \sigma}{\Delta g}
$$


with

$$
(-\Delta g)=\left(\frac{R T}{v_{m}}\right)\left(\ln \frac{f_{b}}{f_{e q}}+\frac{n_{w} v_{w}\left(P-P_{e q}\right)}{R T}\right)
$$

Here, $\sigma$ is the surface tension of NGH in water and is assumed to be equal to that of ice in water; $\Delta g$ is the free energy change per unit volume of NGH; $v_{m}$ is molar volume of the $\mathrm{NGH} ; v_{w}$ is the molar volume of water; and $n_{w}$ is the hydration number.

Some equations above need the value of the film thickness, $y_{L}$, which is obtained using Equation (B.46).

$$
y_{L}=\frac{D_{L} a^{*}}{\left(k_{L} a^{*}\right)}
$$

Other parameters are either directly measured or calculated using published correlations.

\section{B.7. Modification of Englezos et al.'s model}

According to Malegaonkar et al. (1997), there was inconsistency in the Englezos et al.'s model. In writing the initial conditions for the first and second moments of crystal

size distribution ( $\mu_{1}^{0}$ and $\mu_{2}^{0}$ respectively) as shown by Equation (B.41) and (B.42), Englezos et al. considered the crystal size as the crystal diameter $\left(2 r_{c r}\right)$. While in the derivation of the model, they used crystal radius to represent the crystal size. To be consistent, the crystal size should be represented by its diameter too. Then, Malegaonkar et al. made the following modifications on some equations of the Englezos et al.'s model. Equation (B.5) became:

$$
R_{y}(t)=\pi K * \mu_{2}\left(f-f_{e q}\right)
$$

With $s$ represents diameter of the NGH crystal, equation (B.6) became: 


$$
\mu_{2}=\int_{0}^{\infty} s^{2} \phi(s, t) d s
$$

Equation (B.7) became:

$$
D_{L} \frac{d^{2} c}{d y^{2}}=\pi K * \mu_{2}\left(f-f_{e q}\right)
$$

Equation (B.9) became:

$$
D * \frac{d^{2} Y}{d y^{2}}=\pi K * \mu_{2} Y
$$

Equation (B.14) became:

$$
\gamma=y_{L} \sqrt{\frac{\pi K^{*} \mu_{2}}{D^{*}}}
$$

Equation (B.22) became:

$$
(J)_{y=y_{L}} a^{*}=\frac{d}{d t}\left(\frac{c_{w 0} f_{b}}{H^{*}}\right)+\pi K * \mu_{2}\left(f_{b}-f_{e q}\right)
$$

Equation (B.23) became:

$$
\frac{d f_{b}}{d t}=\frac{H^{*} D^{*} \gamma a^{*}}{c_{w 0} y_{L} \sinh \gamma}\left\{\left(f_{g}-f_{e q}\right)-\left(f_{b}-f_{e q}\right) \cosh \gamma\right\}-\frac{\pi K^{*} \mu_{2} H^{*}}{c_{w 0}}\left(f_{b}-f_{e q}\right)
$$

Equation (B.32) became:

$$
G_{a v g}=\left(\frac{1}{L}\right)\left[\int_{0}^{y_{L}}\left(\frac{d s}{d t}\right) d y+\left(\frac{d s}{d t}\right)_{b}\left(L-y_{L}\right)\right]
$$

Equation (B.33) became: 


$$
G=\frac{d s}{d t}=\frac{2 K^{*} M_{H}\left(f-f_{e q}\right)}{\rho_{H}}
$$

Equation (B.34) became:

$$
G_{\text {avg }}=\left(\frac{2 K^{*} M_{H}}{\rho_{H} L}\right)\left[\int_{0}^{y^{L}}\left(f-f_{e q}\right) d y+\left(f_{b}-f_{e q}\right)\left(L-y_{L}\right)\right]
$$

Equation (B.36) became:

$$
G_{\text {avg }}=\left(\frac{2 K * M_{H}}{\rho_{H} L}\right)\left(y_{L} \frac{\left(f_{g}+f_{b}-2 f_{e q}\right)(\cosh \gamma-1)}{\gamma \sinh \gamma}+\left(L-y_{L}\right)\left(f_{b}-f_{e q}\right)\right)
$$




\section{APPENDIX C \\ SKOVBORG AND RASMUSSEN'S MODEL}

Skovborg and Rasmussen's model is the simplification of the Englezos et al.'s model. The model is based on the same reacting system as the one used in the Englezos et al.'s experiments, which is an isothermal-isobaric-semi-batch-stirred-tank-reactor. The bases of the model are as follows (Skovborg and Rasmussen, 1994).

1. In the bulk of water liquid phase, there is equilibrium between dissolved NG and the NGH particles.

2. At gas-liquid interface, there is equilibrium between $\mathrm{NG}$ in the gas phase and the dissolved NG.

3. Dissolved NG is transferred from the gas-liquid interface to the bulk of water liquid according to film theory. This step is the rate determining step in the overall NGH formation process.

Then, the rate of NG consumption from the gas phase is following Equation (C.1) that is the mass transfer rate of the dissolved NG from the gas-liquid interface to the bulk of water liquid.

$$
\frac{d n}{d t}=k_{L} A_{(g-l) c^{c}{ }_{w 0}}\left(x_{i n t}-x_{b}\right)
$$

where $k_{L}$ is the mass transfer coefficient of the dissolved NG in the water liquid film; $A_{(g \text { - }}$ l) is gas-liquid interfacial area; $c_{w 0}$ is the initial concentration of water molecules; $x_{i n t}$ is the mole fraction of the dissolved NG in water (1) phase at the gas-liquid interface in equilibrium with the gas phase at system pressure and temperature; and $x_{b}$ is the mole 
fraction of the dissolved NG in the bulk of water (1) phase in equilibrium with the NGH phase at system pressure and temperature.

$x_{i n t}$ is calculated using the gas solubility data at system pressure and temperature. $x_{b}$ is calculated based on the thermodynamic data of the equilibrium of hydrate-water-gas system. Furthermore, $A_{(g-l)}$ is measured. It leaves $k_{L}$ as the only parameter not known in advance. According to Skovborg and Rasmussen, $k_{L}$ should be measured in an actual hydrate growth system. There is strong possibility that the formation of NGH will affect the value of $k_{L}$ that causes difference from its value obtained from a system where no NGH formed. The existence of NGH in the liquid system will change the hydrodynamic conditions of the liquid phase, which then affects the value of $k_{L}$.

The model can be extended for a system of NG mixtures. If mass transfer of the components is assumed to be independent of each other, then we can extend Equation (C.1) to get Equation (C.2).

$$
\frac{d n_{t o t}}{d t}=\sum_{i=1}^{N G} \frac{d n_{i}}{d t}=c_{w 0} \sum_{i=1}^{N G} k_{L}^{i} A_{(g-l)}\left(x_{i n t}^{i}-x_{b}^{i}\right)
$$

where $n_{\text {tot }}$ is the total moles of NG consumed at a given time; $N G$ is the total number of NG species that formed NGH; and subscript/superscript $i$ represents component $\mathrm{i}$. 


\section{APPENDIX D}

\section{MEASUREMENTS OF THE BULK AND TRUE DENSITIES OF GLASS BEADS}

\section{D.1. Measurement of the bulk density of glass beads}

The procedure of the glass bead bulk density measurement is as follows.

1. Weigh an empty $100 \mathrm{~mL}$ graduated cylinder $\left(=W_{0}\right)$.

2. Add glass beads into the graduated cylinder up to $100 \mathrm{~mL}$.

3. Weigh the graduated cylinder and its content $\left(=W_{l}\right)$.

4. Calculate the bulk density of the glass beads, $\rho_{b}$, using Equation (D.1).

$\rho_{b}=\frac{W_{1}-W_{0}}{100 m L}$

Data of measurements for two different sizes of glass beads are shown in Table D.1. Here, $d_{p}$ is the diameter of glass beads.

TABLE D.1

Measurements of Glass Bead Bulk Density

\begin{tabular}{|c|c|c|c|c|}
\hline \multirow{2}{*}{$d_{p}, \mu \mathrm{m}$} & $W_{0}, \mathrm{~g}$ & $W_{l}, \mathrm{~g}$ & $\rho_{b}, \mathrm{~g} / \mathrm{mL}$ & \multirow{2}{*}{$\rho_{b, \text { avg }, \mathrm{g} / \mathrm{mL}}$} \\
\hline \multirow{3}{*}{240} & 61.7799 & 213.6588 & 1.5188 & \multirow{2}{*}{1.5085} \\
\cline { 2 - 4 } & 61.7799 & 211.0488 & 1.4927 & \\
\cline { 2 - 4 } & 61.7799 & 213.1900 & 1.5141 & \multirow{2}{*}{1.5410} \\
\hline \multirow{3}{*}{510} & 61.7799 & 216.2388 & 1.5446 & \multirow{2}{*}{1.5341} \\
\cline { 2 - 4 } & 61.7799 & 215.1888 & & \\
\cline { 2 - 4 } & 61.7799 & 216.2055 & 1.5443 & \\
& & & & \\
\end{tabular}




\section{D.2. Measurement of the true density of glass beads}

The procedure of the glass bead true density measurement is as follows.

1. Weigh an empty picnometer $\left(=W_{0}\right)$.

2. Fill the picnometer with water whose temperature is known.

3. Weigh the picnometer and its content $\left(=W_{l}\right)$.

4. Find in reference the density of water at the measured temperature $\left(=\rho_{w}\right)$.

5. The volume of the picnometer $\left(V_{p i c}\right)$ is calculated using Equation (D.2).

$$
V_{p i c}=\frac{W_{1}-W_{0}}{\rho_{w}}
$$

6. Weigh a little amount of glass beads $\left(=W_{2}\right)$ and then add it into the picnometer.

7. Add water into the picnometer until the picnometer is fully filled.

8. Weigh the picnometer and its content $\left(=W_{3}\right)$.

9. Calculate the weight of water in the picnometer $\left(=W_{4}\right)$.

$$
W_{4}=W_{3}-W_{0}-W_{2}
$$

10. Calculate the volume of the glass beads in the picnometer $\left(=V_{g b}\right)$.

$$
V_{g b}=V_{p i c}-\frac{W_{4}}{\rho_{w}}
$$

11. Calculate the true density of the glass beads $\left(=\rho_{s}\right)$.

$$
\rho_{s}=\frac{W_{2}}{V_{g b}}
$$

Data of measurements for two different sizes of glass beads are shown in Table

D.2. 
TABLE D.2

Measurements of Glass Bead True Density

\begin{tabular}{|c|c|c|c|c|c|c|c|c|c|c|c|}
\hline $\begin{array}{l}d_{p}, \\
\mu \mathrm{m}\end{array}$ & $W_{0}, \mathrm{~g}$ & $W_{l}, \mathrm{~g}$ & $\begin{array}{l}\mathrm{T}, \\
{ }^{\mathrm{o}} \mathrm{C}\end{array}$ & $\begin{array}{c}\rho_{w} \\
\mathrm{~g} / \mathrm{mL}^{1)}\end{array}$ & $V_{p i c}, \mathrm{~mL}$ & $W_{2}, \mathrm{~g}$ & $W_{3}, \mathrm{~g}$ & $W_{4}, \mathrm{~g}$ & $\begin{array}{l}V_{g b}, \\
\mathrm{~mL}\end{array}$ & $\begin{array}{c}\rho_{s} \\
\mathrm{~g} / \mathrm{mL}\end{array}$ & $\begin{array}{l}\rho_{s, a v g} \\
\mathrm{~g} / \mathrm{mL}\end{array}$ \\
\hline \multirow{3}{*}{240} & 22.6688 & 46.6200 & 28 & 0.996233 & 24.0418 & 21.3288 & 59.3399 & 15.3423 & 8.6415 & 2.4682 & \multirow{3}{*}{2.4694} \\
\hline & 22.6688 & 46.6200 & 28 & 0.996233 & 24.0418 & 20.1099 & 58.6122 & 15.8335 & 8.1484 & 2.4680 & \\
\hline & 22.6688 & 46.6200 & 28 & 0.996233 & 24.0418 & 20.4688 & 58.8399 & 15.7023 & 8.2801 & 2.4721 & \\
\hline \multirow{3}{*}{510} & 22.6688 & 46.6200 & 27 & 0.996513 & 24.0350 & 20.1188 & 58.6522 & 15.8646 & 8.1149 & 2.4792 & \multirow{3}{*}{2.4799} \\
\hline & 22.6688 & 46.6200 & 27 & 0.996513 & 24.0350 & 20.1688 & 58.6888 & 15.8512 & 8.1283 & 2.4813 & \\
\hline & 22.6688 & 46.6200 & 27 & 0.996513 & 24.0350 & 20.4488 & 58.8488 & 15.7312 & 8.2488 & 2.4790 & \\
\hline
\end{tabular}

1) Perry and Green (1985) 


\section{APPENDIX E}

\section{ANALYSIS OF VARIANCE ON DATA OF $K$}

\section{E.1. Analysis of variance (ANOVA) on data of $K$ of MGH experiments of different initial pressures}

To check the effect of initial pressure on $K$, we did ANOVA on data of $K$ of MGH formation as follows. Let $K_{1, j}$ be the data of $K$ for the initial pressure 49.28 bar; $K_{2, j}$ be the data of $K$ for the initial pressure 42.38 bar; and $K_{3, j}$ be the data of $K$ for the initial pressure 45.83 bar. All experiments used the same glass bead size, i.e. $240 \mu \mathrm{m}$. The ANOVA calculation is as follows (Dowdy and Wearden, 1991).

\begin{tabular}{|c|c|c|c|}
\hline & $K_{1, j}$ & $K_{2, j}$ & $K_{3, j}$ \\
\hline & $1.66 \times 10^{-6}$ & $2.43 \times 10^{-6}$ & $1.96 \times 10^{-6}$ \\
\hline & $1.69 \times 10^{-6}$ & $2.32 \times 10^{-6}$ & $1.92 \times 10^{-6}$ \\
\hline & $1.61 \times 10^{-6}$ & $1.64 \times 10^{-6}$ & $2.14 \times 10^{-6}$ \\
\hline & $1.42 \times 10^{-6}$ & & \\
\hline & $1.82 \times 10^{-6}$ & & \\
\hline & $1.98 \times 10^{-6}$ & & \\
\hline & $1.89 \times 10^{-6}$ & & \\
\hline$\sum_{j}\left(K_{i, j}\right)$ & $1.21 \times 10^{-5}$ & $6.39 \times 10^{-6}$ & $6.02 \times 10^{-6}$ \\
\hline$\overline{\sum_{j}\left(K_{i, j}\right)^{2}}$ & $2.10 \times 10^{-11}$ & $1.40 \times 10^{-11}$ & $1.21 \times 10^{-11}$ \\
\hline$\overline{\left(\sum_{j}\left(K_{i, j}\right)\right)^{2}}$ & $1.46 \times 10^{-10}$ & $4.08 \times 10^{-11}$ & $3.62 \times 10^{-11}$ \\
\hline$K_{i, \text { average }}$ & $1.72 \times 10^{-6}$ & $2.13 \times 10^{-6}$ & $2.01 \times 10^{-6}$ \\
\hline
\end{tabular}




$$
\begin{aligned}
& N=\Sigma_{i}\left(n_{i}\right)=13 \\
& T=\Sigma_{i} \Sigma_{j}\left(K_{i, j}\right)^{2}=4.71 \times 10^{-11} \\
& A=\Sigma_{i}\left\{\left(\Sigma_{j}\left(K_{i, j}\right)\right)^{2} / n_{i}\right\}=4.65 \times 10^{-11} \\
& C F=\left\{\sum_{i} \Sigma_{j}\left(K_{i, j}\right)\right\}^{2} / N=4.61 \times 10^{-11}
\end{aligned}
$$

\begin{tabular}{|c|c|c|c|c|c|}
\hline Source & $d f$ & $S S$ & $M S=S S / d f$ & $F=M S_{a} / M S_{e}$ & $F_{0.05,2,10}$ \\
\cline { 1 - 4 } Among groups & 2 & $A-C F=4.05 \times 10^{-13}$ & $M S_{a}=2.03 \times 10^{-13}$ & 3.34 & 4.103 \\
\hline Error & 10 & $T-A=6.07 \times 10^{-13}$ & $M S_{e}=6.07 \times 10^{-14}$ & & \\
\hline
\end{tabular}

Hence, $F<F_{0.05,2,10}$

Conclusion : there was no significant difference among $K$ values of different initial pressures.

\section{E.2. Analysis of variance (ANOVA) on data of $K$ of MGH experiments of different glass bead sizes}

To check the effect of glass bead size on $K$, we did ANOVA on data of $K$ of MGH formation as follows. Let $K_{1, j}$ be the data of $K$ of glass beads $240 \mu \mathrm{m}$; and $K_{2, j}$ be the data of $K$ of glass beads $510 \mu \mathrm{m}$. Both experiments were done at the same initial pressure, i.e. 49.28 bar. The ANOVA calculation is as follows (Dowdy and Wearden, 1991). 


\begin{tabular}{|l|c|c|}
\hline & $K_{l, j}$ & $K_{2, j}$ \\
\hline & $1.66 \times 10^{-6}$ & $2.46 \times 10^{-6}$ \\
\hline & $1.69 \times 10^{-6}$ & $1.46 \times 10^{-6}$ \\
\hline & $1.61 \times 10^{-6}$ & $1.69 \times 10^{-6}$ \\
\hline & $1.42 \times 10^{-6}$ & $2.45 \times 10^{-6}$ \\
\hline & $1.82 \times 10^{-6}$ & $1.66 \times 10^{-6}$ \\
\hline & $1.98 \times 10^{-6}$ & $1.15 \times 10^{-5}$ \\
\hline$\Sigma_{j}\left(K_{i, j}\right)$ & $1.89 \times 10^{-6}$ & $2.29 \times 10^{-11}$ \\
\hline$\Sigma_{j}\left(K_{i, j}\right)^{2}$ & $1.21 \times 10^{-5}$ & $1.32 \times 10^{-10}$ \\
\hline$\left(\Sigma_{j}\left(K_{i, j}\right)\right)^{2}$ & $2.10 \times 10^{-11}$ & $1.92 \times 10^{-6}$ \\
\hline$K_{i, \text { average }}$ & $1.46 \times 10^{-10}$ & \\
\hline
\end{tabular}

$$
\begin{aligned}
& N=\Sigma_{i}\left(n_{i}\right)=13 \\
& T=\Sigma_{i} \Sigma_{j}\left(K_{i, j}\right)^{2}=4.40 \times 10^{-11} \\
& A=\Sigma_{i}\left\{\left(\Sigma_{j}\left(K_{i, j}\right)\right)^{2} / n_{i}\right\}=4.28 \times 10^{-11} \\
& C F=\left\{\Sigma_{i} \Sigma_{j}\left(K_{i, j}\right)\right\}^{2} / N=4.27 \times 10^{-11}
\end{aligned}
$$

\begin{tabular}{|c|c|c|c|c|c|}
\hline Source & $d f$ & $S S$ & $M S=S S / d f$ & $F=M S_{d} / M S_{e}$ & $F_{0.05,1,11}$ \\
\hline Among groups & 1 & $A-C F=1.18 \times 10^{-13}$ & $M S_{a}=1.18 \times 10^{-13}$ & 1.134 & 4.844 \\
\cline { 1 - 4 } Error & 11 & $T-A=1.14 \times 10^{-12}$ & $M S_{e}=1.04 \times 10^{-13}$ & & \\
\hline
\end{tabular}

Hence, $F<F_{0.05,1,11}$ 
Conclusion : there was no significant difference among $K$ values of different glass bead sizes.

\section{E.3. Average value of $K$ of MGH formation}

Because there was no significant effect of both initial pressure and glass bead size on $K$, the average value of $K$ was obtained using all data of $K$. The calculation is as follows.

Grand mean $=\Sigma_{i} \Sigma_{j}\left(K_{i, j}\right) / N=1.89 \times 10^{-6}$

Variance $=\left\{\Sigma_{i} \Sigma_{j}\left(K_{i, j}\right)^{2}-\left(\Sigma_{i} \Sigma_{j}\left(K_{i, j}\right)\right)^{2} / N\right\} /(N-1)=1.08 \times 10^{-13}$

$t_{0.025,18}=2.101$

$t_{0.025,18}(\text { Variance } / N)^{0.5}=0.16 \times 10^{-6}$

We obtained, $K_{\text {average }}=(1.89 \pm 0.16) \times 10^{-6} \mathrm{~m} / \mathrm{s}$

\section{E.4. Analysis of variance (ANOVA) on data of $K$ of PGH experiments of different initial pressures}

To check the effect of initial pressure on $K$ of PGH formation, we did ANOVA test as follows. Let $K_{l, j}$ be the data of $K$ of the initial pressure 4.32 bar; and $K_{2, j}$ be the

data of $K$ of the initial pressure 4.18 bar. All experiments used the same glass bead size, i.e. $240 \mu \mathrm{m}$. The ANOVA calculation is as follows (Dowdy and Wearden, 1991). 


\begin{tabular}{|l|c|c|}
\hline & $K_{1, j}$ & $K_{2, j}$ \\
\hline & $2.36 \times 10^{-6}$ & $2.12 \times 10^{-6}$ \\
\hline & $1.81 \times 10^{-6}$ & $2.99 \times 10^{-6}$ \\
\hline$\Sigma_{j}\left(K_{i, j}\right)$ & $2.58 \times 10^{-6}$ & $1.61 \times 10^{-6}$ \\
\hline$\Sigma_{j}\left(K_{i, j}\right)^{2}$ & $6.75 \times 10^{-6}$ & $6.72 \times 10^{-6}$ \\
\hline$\left(\Sigma_{j}\left(K_{i, j}\right)\right)^{2}$ & $1.55 \times 10^{-11}$ & $1.60 \times 10^{-11}$ \\
\hline$K_{i, \text { average }}$ & $4.56 \times 10^{-11}$ & $4.52 \times 10^{-11}$ \\
\hline
\end{tabular}

$$
\begin{aligned}
& N=\Sigma_{i}\left(n_{i}\right)=6 \\
& T=\Sigma_{i} \Sigma_{j}\left(K_{i, j}\right)^{2}=3.15 \times 10^{-11} \\
& A=\Sigma_{i}\left\{\left(\Sigma_{j}\left(K_{i, j}\right)\right)^{2} / n_{i}\right\}=3.0240300 \times 10^{-11} \\
& C F=\left\{\Sigma_{i} \Sigma_{j}\left(K_{i, j}\right)\right\}^{2} / N=3.0240150 \times 10^{-11}
\end{aligned}
$$

\begin{tabular}{|c|c|c|c|c|c|}
\hline Source & $d f$ & $S S$ & $M S=S S / d f$ & $F=M S_{a} / M S_{e}$ & $F_{0.05,1,4}$ \\
\hline Among groups & 1 & $A-C F=1.50 \times 10^{-16}$ & $M S_{a}=1.50 \times 10^{-16}$ & 0.0005 & 7.709 \\
\cline { 1 - 4 } Error & 4 & $T-A=1.29 \times 10^{-12}$ & $M S_{e}=3.22 \times 10^{-13}$ & & \\
\hline
\end{tabular}

Hence, $F<F_{0.05,1,4}$

Conclusion : there was no significant difference among $K$ values of different initial pressures. 


\section{E.5. Average value of $K$ of PGH formation}

Because there was no significant effect of initial pressure on $K$, the average value of $K$ was obtained using all data of $K$. The calculation is as follows.

Grand mean $=\sum_{i} \Sigma_{j}\left(K_{i, j}\right) / N=2.25 \times 10^{-6}$

Variance $=\left\{\sum_{i} \Sigma_{j}\left(K_{i, j}\right)^{2}-\left(\sum_{i} \Sigma_{j}\left(K_{i, j}\right)\right)^{2} / N\right\} /(N-1)=2.58 \times 10^{-13}$

$t_{0.025,5}=2.571$

$t_{0.025,5}(\text { Variance } / N)^{0.5}=0.53 \times 10^{-6}$

We obtained, $K_{\text {average }}=(2.25 \pm 0.53) \times 10^{-6} \mathrm{~m} / \mathrm{s}$ 